\title{
Theorie und Praxis von Technology Governance
}

Stephan Bröchler, Georg Aichholzer, Petra Schaper-Rinkel

(Hrsg.) 



\title{
Theorie und Praxis von Technology Governance
}

\author{
Stephan Bröchler', Georg Aichholzer², \\ Petra Schaper-Rinkel ${ }^{3}$ (Hrsg.) \\ I Institut für Politikwissenschaft, Universität Duisburg-Essen \\ ${ }^{2}$ ITA - Institut für Technikfolgen-Abschätzung, Österreichische Akademie der Wissenschaften \\ ${ }^{3}$ AIT - Austrian Institute of Technology $\mathrm{GmbH}$
}

Mit Beiträgen von:

\section{Armin Grunwald}

Institut für Technikfolgenabschätzung und Systemanalyse (ITAS), Karlsruhe

\section{Georg Simonis}

Institut für Politikwissenschaft, FernUniversität in Hagen

\section{Marc Mölders}

Institut für Wissenschafts- und Technikforschung (IWT), Universität Bielefeld

\section{Urte Brand und Arnim von Gleich}

Fachgebiet Technikgestaltung und Technologieentwicklung, Universität Bremen

\section{Gerhard Fuchs}

Abteilung für Organisations- und Innovationssoziologie, Universität Stuttgart 


\section{Inhalt}

Stephan Bröchler, Georg Aichholzer, Petra Schaper-Rinkel

Einleitung: Von Governance zu Technology Governance ....................................................... 5

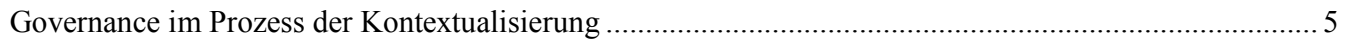

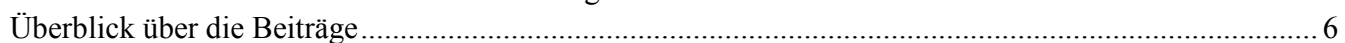

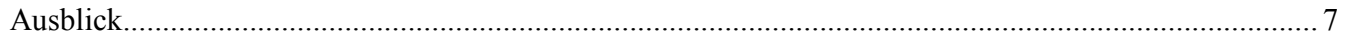

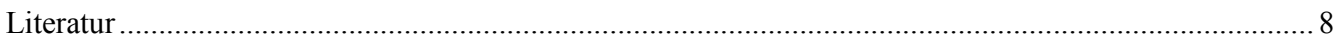

Armin Grunwald

Responsible Innovation: Neuer Ansatz der Gestaltung von Technik

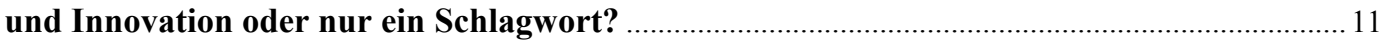

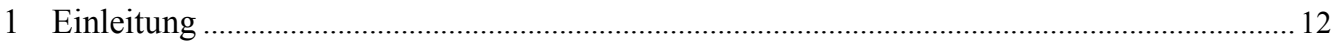

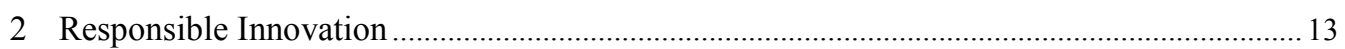

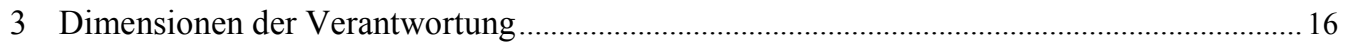

4 Vorläufer der „Responsible Innovation“ ..................................................................................... 19

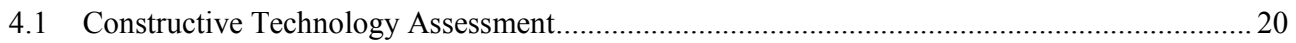

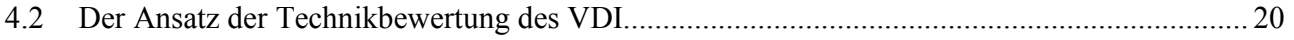

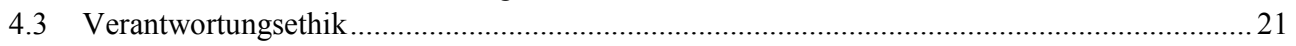

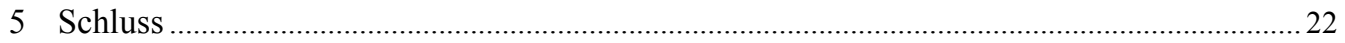

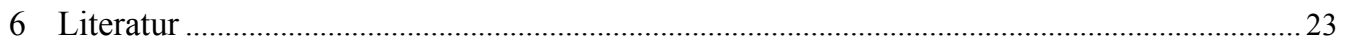

Georg Simonis

Technikfolgenabschätzung als Ressource von Technology Governance ...................................25

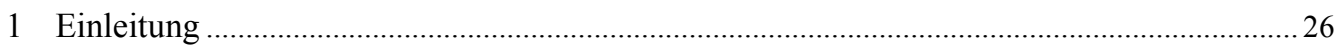

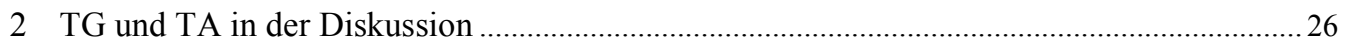

3 TG differenzierungstheoretisch betrachtet ............................................................................... 28

4 Politische Technology Governance ........................................................................................... 31

5 TA als Ressource für Technology Governance ................................................................... 33

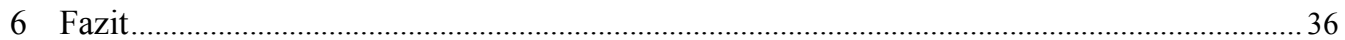

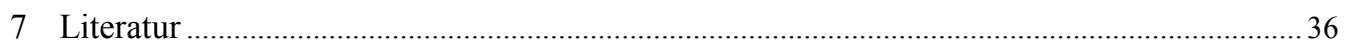

Marc Mölders

Trotz aller Differenzen - Theoretische und empirische Perspektiven auf das Hummel-Paradox der (Technology-)Governance ......................................................... 39

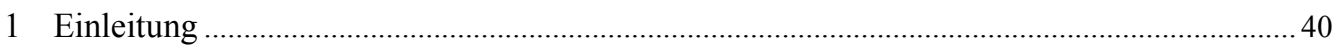

2 Gelingende Governance: Zur Unterscheidung einer operativen und einer integrativen Leistungsdimension am Beispiel der ,von Wedel-Kommission“....................... 41

3 Theoretische Entfaltungen des Hummel-Paradoxes: Differenzen und

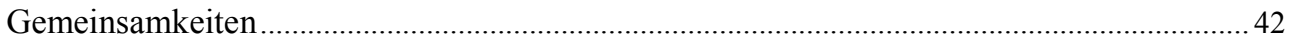

4 Empirische Perspektiven auf das Hummel-Paradox: Der Fall des „Single European Sky“..... 45

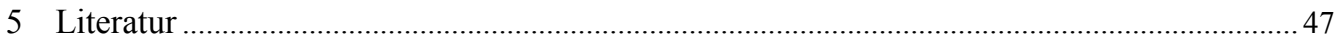


Urte Brand, Arnim von Gleich

Leitorientierte Technologiegestaltung als Beitrag zur Umsetzung des Vorsorgeprinzips.

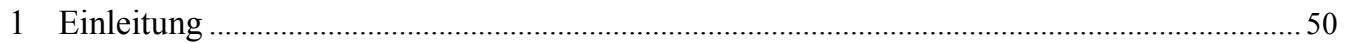

2 Möglichkeiten der Gestaltung und Richtungsgebung von technologischen

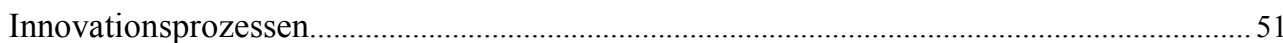

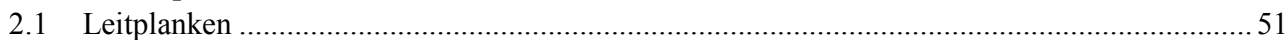

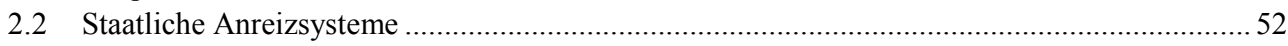

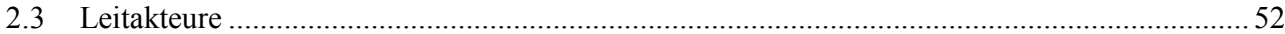

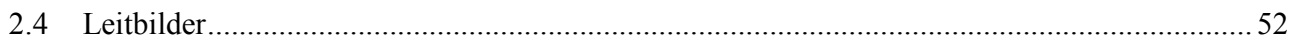

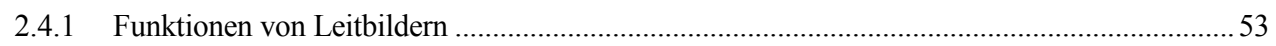

2.4.2 Differenzierung von Leitbildern - Dreiebenenmodell ..........................................................53

3 Retrospektive Analyse von erfolgreichen Leitkonzepten in systemischen

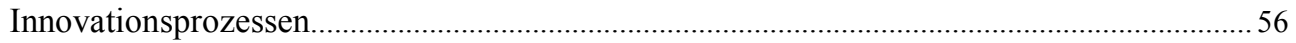

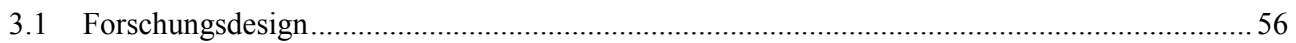

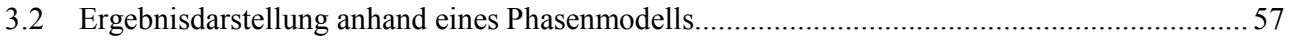

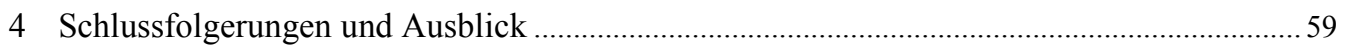

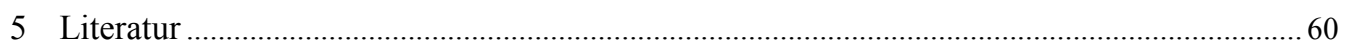

Gerhard Fuchs

Zur Governance von technologischen Innovationen im Energiesektor ......................................6 65

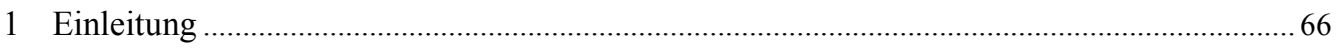

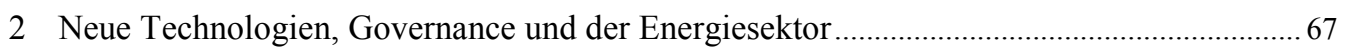

3 Die Förderung von CCS in Deutschland und Norwegen ...................................................... 67

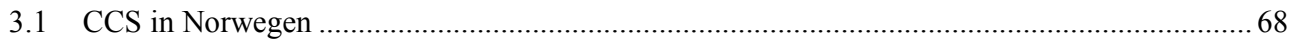

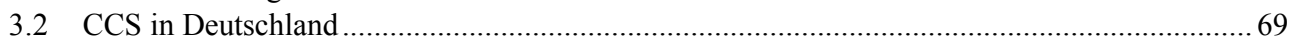

4 Die Förderung von Photovoltaik in Deutschland und Japan ................................................... 71

4.1 Photovoltaik (PV)-Entwicklung in Japan ................................................................................ 71

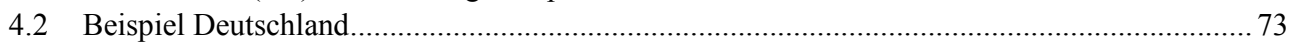

5 Fazit: Governance von Innovationen im Energiesektor ......................................................... 76

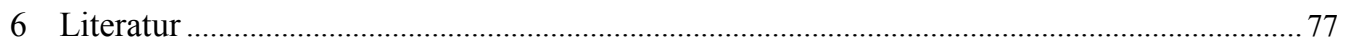

Die Beiträge dieser Sondernummer basieren auf Vorträgen, die unter Beteiligung des ITA im Rahmen des Netzwerks TA auf einem Workshop der Arbeitsgruppe ,,Technikfolgenabschätzung und Governance" am 21. und 22.11.2011 in Berlin gehalten wurden. 


\section{IMPRESSUM}

\section{Medieninhaber:}

Österreichische Akademie der Wissenschaften

Juristische Person öffentlichen Rechts (BGBI 569/|921 idF BGBI I | 30/2003)

Dr. Ignaz Seipel-Platz 2, A- 1010 Wien

\section{Herausgeber:}

Institut für Technikfolgen-Abschätzung (ITA)

Strohgasse 45/5, A- 1030 Wien

http://www.oeaw.ac.at/ita

Die ITA-manu:scripts erscheinen unregelmäßig und dienen der Veröffentlichung

von Arbeitspapieren und Vorträgen von Institutsangehörigen und Gästen.

Die manu:scripts werden ausschließlich über das Internetportal „epub.oeaw“

der Öffentlichkeit zur Verfügung gestellt:

http://epub.oeaw.ac.at/ita/ita-manuscript

ITA-manuscript Nr.: ITA-12-02_Sondernummer (September/20 I2)

ISSN-online: I 8|8-6556

http://epub.oeaw.ac.at/ita/ita-manuscript/ita_12_02_Sondernummer.pdf

c 2012 ITA - Alle Rechte vorbehalten 


\title{
Einleitung: Von Governance zu Technology Governance
}

\author{
Stephan Bröchler, Georg Aichholzer, Petra Schaper-Rinkel
}

Governance stellt eine Innovation für das Verständnis der Regelung kollektiver Sachverhalte in entwickelten komplexen Gesellschaften dar. Innovativ sind dabei vor allem zwei Aspekte: Mit Governance verbindet sich eine veränderte Realität der Tätigkeit wie auch der Art und Weise des Regierens mit einer neuen Sichtweise auf politische Handlungskoordinierung (Benz/Dose 2010). „Neu“ ist Governance dabei nicht im Sinne eines Paradigmenwechsels im Verständnis von Thomas S. Kuhn (Kuhn 2001). Vertreter des Governance-Ansatzes sind keine Kopernikaner und die Kritiker keine Ptolemäer. Die Innovation der Governance-Perspektive lässt sich angemessener als Perspektivwechsel beschreiben. Neu sind an diesem Zugang zwei Aspekte: Erstens, dass staatliche und konfliktfähige nicht-staatliche Akteure in die Bearbeitung öffentlicher Probleme immer häufiger einbezogen werden, um politische Handlungsfähigkeit zu erhöhen. Zweitens die Entwicklung, dass die Verhandlungssysteme, in denen sich Governance vollzieht, nun systematisch Gegenstand der Forschung werden.

\section{Governance im Prozess der Kontextualisierung}

Doch mit der „Entdeckung“ von Governance ist zunächst lediglich das Potential für eine weitergehende notwendige theoretisch wie empirisch gehaltvolle Analyse des Wandels von Staatlichkeit erarbeitet. Spannend wird Governance im nachfolgenden Prozess der forschungsbasierten und diskursiven Kontextualisierung. Denn erst in der kritischen, vielstimmigen Auseinandersetzung und in der Einbettung in unterschiedliche disziplinäre Erkenntnisinteressen sowie Forschungsfelder muss sich erweisen, welchen Ertrag die Governance-Sicht konkret zu leisten vermag. Hier zeigt sich sehr deutlich, dass Governance aus analytischer Sicht ein ergebnisoffenes Analysekonzept darstellt. Es beinhaltet die Ausleuchtung von Aufgabenerfüllung, Leistungserbringung und Problemlösung dieser Verhandlungssysteme wie auch die Einbeziehung der Aspekte Gewinn und Erhalt von politischer Macht (Mayntz 2010).

Der Verlauf der Debatte wie die Ausdifferenzierung der Forschungsinteressen zeigen, wie intensiv und lebhaft sich die Kontextualisierung des Governance-Begriffs in den letzten Jahren entwickelt. Zum Ausdruck kommt die Kontextualisierung erstens in Form horizontaler Ausdifferenzierung. In den letzten Jahren nimmt die Zahl der Felder zu, in denen Governance-Prozesse identifiziert werden. $\mathrm{Zu}$ nennen sind hier beispielsweise Global Governance, Local Governance, Multilevel-Governance, Corporate Governance, Regional Governance, Educational Governance, IT-Governance, RiskGovernance oder Technology Governance. Ob die „Governance-Konjunktur“ in allen Fällen Ausdruck solider Forschung oder zuweilen doch Modeerscheinung im Sinne einer „Governance-Hype“ ist, wird sich jedoch weniger an der Anzahl als an der Qualität der Forschungsbeiträge zu den einzelnen Feldern entscheiden. Denn die Kontextualisierung vollzieht sich neben der horizontalen auch als vertikale Differenzierung der Governanceforschung. Hier geht es um die Frage, ob und wie sich die Kontextualisierung innerhalb eines Governance-Forschungsfeldes entwickelt.

Als ein interessantes Beispiel für eine ertragreiche Kontextualisierung von Governance erweisen sich die Entwicklungen im Bereich der Technikfolgen-Abschätzung (TA). Dabei kristallisiert sich in den letzten Jahren besonders das „Netzwerk Technikfolgen-Abschätzung“ („Netzwerk TA“) als ein wichtiger Ort, in dem der Beitrag der Governance-Perspektive für TA intensiv und kontinuierlich diskutiert wird (Bora/Decker/Grunwald/Renn 2005; Bora/Bröchler/Decker 2007; Aichholzer/ 
Bora/Bröchler/Decker/Latzer 2010). Es wird argumentiert, dass TA eine eigene Governance-Struktur darstellt (Bröchler 2005: 348 ff.; Bröchler 2007: 417 ff). Die unterschiedlichen institutionalisierten TA-Ansätze, wie strategische, konstruktive, parlamentarische, partizipative und deliberative TA lassen sich insgesamt als problembearbeitender Regelungsmechanismus begreifen (Simonis 2001). Hier arbeiten je nach Ansatz in unterschiedlich konfigurierten Verhandlungssystemen staatliche, wissenschaftliche und zunehmend auch zivilgesellschaftliche Akteure bei der Lösung technikbezogener gesellschaftlicher Probleme zusammen.

Als eine weitere Phase der Kontextualisierung des Governance-Verständnisses für TechnikfolgenAbschätzung lässt sich die jüngste Debatte um „Technology Governance“ verstehen. Ein Begriff, der in jüngerer Zeit mehr und mehr für Governance-Formen im Bereich der Technik zur Anwendung kommt. Technology Governance läßt sich als Zusammenwirken zivilgesellschaftlicher Akteure (Unternehmen, Wissenschaft) und staatlicher Akteure bei der Entwicklung und Nutzung technischer Innovationen beschreiben.

Im Jahr 2008 befasste sich das „Netzwerk TA“ auf seiner dritten internationalen Konferenz in Wien intensiv mit dem Thema Technology Governance (TG) und seiner Bedeutung für TA (Aichholzer/Bora/Bröchler/Decker/Latzer 2010): Wie sind TA und TG aufeinander bezogen? Erste konzeptionelle Klärungsansätze formulierten Stefan Kuhlmann (Kuhlmann 2010, S. 41 ff.) und Stephan Bröchler (Bröchler 2010, S. 63 ff.). Beide kamen zu dem Schluss, dass es sinnvoll ist, TA und TG als Bereiche zu trennen, um Hinweise auf Spielräume für die Gestaltung sozio-technischer Systeme besser identifizieren und nutzen zu können. Kuhlmann interpretiert TA und TG als zwei getrennte Handlungsfelder: zum einen die de facto Governance sozio-technischer Systeme zum anderen TA als Instrument reflexiver Governance (Kuhlmann 2010, S. 48). Bröchler plädiert dafür, zwei Begriffsverwendungen zu unterscheiden. Erstens eine prima facie normative Verwendung des Begriffs. Zweitens eine Verwendung des Konzepts als analytische Forschungsstrategie. Dabei komme TA die Funktion der Brücke zwischen dem normativen Verständnis und der Interpretation als Forschungsstrategie zu (Bröchler 2010, S.70).

Die folgenden Beiträge dokumentieren die Erträge des Workshops der Arbeitsgruppe „Technikfolgenabschätzung und Governance" des Netzwerks TA in Berlin vom 21.11.2011 bis 22.11.2011. Das Thema der von Stephan Bröchler, Petra Schaper-Rinkel und Claus Seibt organisierten Tagung lautete: „Theorie und Praxis von Technology Governance. Fragestellungen und Erkenntnisse aktueller Forschung im Kontext Technology Assessment und Governance“. Technology Governance als neue Praxis des Regierens wie auch als analytisches Konzept, diese neuen Formen des Regierens in den Blick zu nehmen, standen im Zentrum des Workshops. Ziel war es, einen Beitrag zu leisten, die wechselseitige Relevanz von TA und TG in konzeptioneller wie empirischer Sicht genauer auszuleuchten. Dabei sollten nicht nur Veränderungen in Form neuer Technologien in den Blick genommen, sondern auch die eigenen Praxen der TA analysiert werden.

\section{Überblick über die Beiträge}

Die Beiträge verdeutlichen zum einen die Pluralität der Vortragsthemen im Rahmen der Fragestellung des Workshops: „Responsible Innovation“ (Armin Grunwald); „TA als Wissensressource“ (Georg Simonis); „Hummel Paradox“ (Marc Mölders); „Leitorientierte Technologiegestaltung“ (Urte Brand und Arnim von Gleich) und „Technologische Innovationen im Energiesektor“ (Gerhard Fuchs).

Zum anderen zeigt sich bei aller Heterogenität, dass allen Beiträgen der Aspekt der Gestaltung soziotechischer Innovationen im Kontext von Technology Governance und Technikfolgen-Abschätzung wie ein „roter Faden“ eingewebt ist. 
Im Beitrag von Armin Grunwald spielen normative Aspekte der Gestaltung von Technik eine zentrale Rolle. Gegenstand ist die kritische Auseinandersetzung mit Responsible Innovation, als dem derzeit wohl weitestgehenden Versuch in der Tradition von Constructive TA, sozio-technische Systeme im Blick auf nachhaltige und verantwortliche Technik erfolgreich zu gestalten. Trotz einer Reihe gut begründeter Kritikpunkte und Einwände plädiert Grunwald dafür, den Ansatz der Responsible Innovation nicht zu verwerfen. Im Gegenteil: Es gelte vielmehr, durch konzeptionelle Analyse, Praxis und reflektierendes Lernen Responsible Innovation zu formen und inhaltlich zu füllen.

Georg Simonis setzt sich explizit mit dem Verhältnis von TG und TA mit dem Ziel auseinander, Wege zu finden, wie TA-Angebote verbessert und die Leistungsfähigkeit von TG erhöht werden kann. TA stelle ein normales Element von TG dar. In der Auseinandersetzung mit Konzeptionalisierungen des Verhältnisses von TA und TG plädiert er für ein Verständnis, in dem sich normative und reale Aspekte der Entwicklung analytisch nicht trennen lassen. Aus einer differenzierungstheoretischen Perspektive kommt Simonis zu dem Schluss, dass TA eine Wissensressource darstellt, die zur Steigerung der Leistungsfähigkeit von TG genutzt werden kann.

Marc Mölders setzt sich mit der Prämisse auseinander, dass erfolgreiche Technology Governance gelingende Verhandlungssysteme voraussetzt. Dies erscheine auf den ersten Blick jedoch als so unmöglich, wie die Fähigkeit von Hummeln, aufgrund der Gesetze der Aerodynamik, zu fliegen. Dennoch, so Mölders, vermögen Hummeln zu fliegen und Verhandlungssysteme trotz der Geschlossenheit und Eigensinnigkeit sozialer Systeme zu gelingen. Der Schlüssel zur Auflösung des Hummel-Paradoxes der Technology Governance wird im Modell der Konversationskreise identifiziert. Die Analyse von Mölders ist besonders im Blick auf normative TA-Ansätze wie Constructive TA und Anticipatory TA spannend.

Urte Brand und Arnim von Gleich formulieren zunächst ein klassisches Dilemma, vor das TA gestellt sei. Setzt TA am Beginn des Innovationsprozesses an, sei die Gestaltungsfähigkeit von Technik zwar sehr hoch, jedoch das Wissen über Risiken und Folgen noch (zu) gering. Beginnt TA am Ende des Innovationsprozesses, sei das Wissen deutlich besser, aber die Gestaltungsmöglichkeiten ,at the end of the pipe“ (zu) gering. In dieser Situation wird der Leitbildansatz als ,dritter Weg“" diskutiert, um die Gestaltung von Innovationsprozessen zu stärken. Besonders das Leitkonzept „Energieautarkie“ wird als ,,best practice“ dargelegt und anhand von Fallbeispielen dokumentiert, um zu zeigen, dass mit Hilfe von Leitorientierungen Innovationsprozesse erfolgreich gestaltet werden können.

Gerhard Fuchs hebt in seinem Beitrag die institutionalistische Orientierung der Governance-Forschung heraus. Governance fixiere sich auf die Analyse der Beschaffenheit von Regelungsstrukturen und blende die Erforschung der Veränderung existierender Strukturen aus. Diese werden im Rahmen institutionalistischer und evolutionstheoretischer Ansätze prima facie durch externe Faktoren wie Krisen und Schocks erklärt. Demgegenüber geht Fuchs in seinem Beitrag am Beispiel des Energiesektors der Frage nach, welche Bedeutung Akteuren, Strategien und Machtkonstellationen für die Veränderung sozio-technischer Systeme als interne Faktoren von Veränderung zukommt.

\section{Ausblick}

Die Publikation der Tagungsbeiträge ist Ausgangspunkt für weitere Aktivitäten des Netzwerks TA und seiner Arbeitsgruppe zum Thema. Auf dem Workshop in Berlin 2011 wurden eine Reihe von interessanten Vorschlägen auf die Agenda der zukünftigen Arbeit der AG „Governance und Technikfolgenabschätzung“" gesetzt:

Wie tragen unterschiedliche TA-Verfahren (wie strategische, konstruktive, parlamentarische, partizipative, deliberative TA-Ansätze) zu Technology Governance bei? 
Was bedeutet es für die Zukunft von TA, wenn TA nicht nur analytische Praxis sondern auch selbst Teil der Technology Governance ist? Diese Fragen sind von steigender Bedeutung, da gerade an der Schnittstelle zwischen TA und den Science and Technology Studies (STS) Methoden entwickelt wurden, die praktisch-politisch darauf zielen, die Technologie-Entwicklung direkt interaktiv zu beeinflussen (Rip/Schot 1997; Guston/Sarewitz 2002; Rip 2008; Robinson 2009; Roco et al. 2010: 442; Roco et al. 2011; von Schomberg 2011; European Commission 2012; Schaper-Rinkel 2012 forthcoming).

Welchen Beitrag können Governance-Strategien im Umgang mit globalen und vernetzten Risiken leisten? Zu denken ist etwa an steigende Treibhausgasemissionen, Cyberangriffe oder Wasserversorgungskrisen. Risk Governance ist ein interessanter Ansatz, der diese Fragen thematisiert und die Frage beantworten möchte, ob und wie Politik Handlungsfähigkeit erzielen kann (Renn 2008; Aven/Renn 2010; Beisheim/Rudloff/Ulmer 2012).

Zum Schluss möchten wir uns ganz herzlich bei den Autorinnen und Autoren bedanken, die bereit waren, ihre Vortragsmanuskripte für die Publikation zu überarbeiten. Herzlichen Dank an Claus Seibt für die - wie stets - sehr gute Zusammenarbeit bei der Konzeption des Workshops. Ein besonderer Dank gilt Liudger Dienel und dem Team des ZTG, das die Durchführung des Workshops in Berlin erst ermöglichte. Last but not least bedanken wir uns beim Institut für Technikfolgenabschätzung in Wien für die Möglichkeit, im Rahmen der Reihe „ITA-Manu-scripte“ eine Sondernummer zu publizieren und für die tatkräftige Unterstützung bei der Erstellung der Druckfassung durch Manuela Kaitna.

\section{Literatur}

Aichholzer, G., Bora, A., Bröchler, S., Decker, M., Latzer, M. (Hrsg.), 2010, Technology Governance. Der Beitrag der Technikfolgenabschätzung. Berlin: Edition Sigma.

Aven, T., Renn, O., 2010, Risk Management an Governance. Concepts, Guidelines and Applications, Berlin: Springer.

Beisheim, M., Rudloff, B. Ulmer, K. 2012, Risiko-Governance: Umgang mit globalen und vernetzten Risiken. Arbeitspapier FG 8, 2012/1 Februar 2012. Stiftung Wissenschaft und Politik, Berlin.

Benz, A., Dose, N. (Hrsg.), 2010, Governance - Regieren in komplexen Regelsystemen: Eine Einführung. 2. Auflage. Wiesbaden: VS.

Benz, A., Dose, N., 2010, Governance - Modebegriff oder nützliches sozialwissenschaftliches Konzept? In: Benz, A., Dose, N. (Hrsg.), Governance - Regieren in komplexen Regelsystemen: Eine Einführung. 2. Auflage. Wiesbaden: VS, S.13-36.

Bora, A., Bröchler, S., Decker, M. (Hg.), 2007, Technology Assessment in der Weltgesellschaft. Berlin: Edition Sigma.

Bora, A., Decker, M., Grunwald, A., Renn, O. (Hg.), 2005, Technik in einer fragilen Welt. Die Rolle der Technikfolgenabschätzung. Berlin: Edition Sigma.

Bröchler, S., 2010, Technikfolgenabschätzung und Technology Governance. In: Aichholzer, G., Bora, A., Bröchler, S., Decker, M., Latzer, M. (Hg.): Technology Governance. Der Beitrag der Technikfolgenabschätzung. Berlin: Edition Sigma, S. 63-74. 
Bröchler, S., 2005, Governance in der Technikfolgenabschätzung. In: Bora, A., Decker, M., Grunwald, A., Renn, O. (Hg.), 2005, Technik in einer fragilen Welt. Die Rolle der Technikfolgenabschätzung. Berlin: Edition Sigma, S. 341-352.

Bröchler, S., 2001, Technik. In: Benz, A., Lütz, S., Schimank, U., Simonis, G. (Hrsg.): Handbuch Governance.Theoretische Grundlagen und empirische Anwndungsfelder. Wiesbaden: VS, S. 413-423.

European Commission, 2012, Ethical and regulatory challenges to science and research policy at the global level. Publications Office of the European Commission, Luxembourg.

Guston, David H., Sarewitz, D., 2002, Real-time technology assessment. In: Technology in Society 24, S. 93-109.

Kuhlmann, S., 2010, TA als Tanz: Zur Governance technologischer Innovation. Neue Aufgaben des Technology Assessment. In: Aichholzer, G., Bora, A., Bröchler, S., Decker, M., Latzer, M. (Hg.): Technology Governance. Der Beitrag der Technikfolgenabschätzung. Berlin: Edition Sigma, S. 41-57.

Kuhn, T. S., 2001, Die Struktur wissenschaftlicher Revolutionen. Frankfurt: Suhrkamp.

Mayntz, R., 2010, Governance im modernen Staat. In: Benz, A., Dose, N. (Hrsg.), Governance Regieren in komplexen Regelsystemen: Eine Einführung. 2. Auflage. Wiesbaden, S. 37-48.

Renn, O., 2008, Risk Governance: Coping with Uncertainty in a complex World. London: Earthscan.

Rip, A., 2008, Nanoscience and Nanotechnologies: Bridging Gaps Through Constructive Technology Assessment. In: Hirsch Hadorn, G.; Hoffmann-Riem, H.; Biber-Klemm, S.; Grossenbacher-Mansuy, W.; Joye, D.; Pohl, C.; Wiesmann, U.; Zemp, E. (Hrsg.): Handbook of Transdisciplinary Research, S. 145-157.

Rip, A., Schot, J., 1997, The past and future of constructive technology assessment. In: Technological Forecasting and Social Change 54, S. 251-268.

Robinson, D. K. R., 2009, Co-evolutionary scenarios: An application to prospecting futures of the responsible development of nanotechnology. In: Technological Forecasting and Social Change 76, S. 1222-1239.

Roco, M. C., Harthorn, B., Guston, D., Shapira, P., 2011, Innovative and responsible governance of nanotechnology for societal development. In: Journal of Nanoparticle Research 13, S. 3557-3590.

Roco, M. C., Mirkin, C. A., Hersam, M., 2010, Nanotechnology Research Directions for Societal Needs in 2020. Retrospective and Outlook. Berlin/Boston: Springer.

Schaper-Rinkel, P., 2012 (forthcoming), The role of future-oriented technology analysis in the governance of emerging technologies: The example of nanotechnology. In: Technological Forecasting and Social Change.

Simonis, G., 2001, Die TA-Landschaft in Deutschland - Potentiale refelxiver Techniksteuerung. In: Simonis, G., Martinsen, R., Saretzki, T. (Hg.): Politik und Technik - Analysen zum Verhältnis von technologischem, politischem und staatlichem Wandel am Anfang des 21. Jahrhunderts (PVS-Sonderheft 31/2000). Wiesbaden: VS, S. 425-456.

von Schomberg, R., (Hrsg.) 2011, Towards Responsible Research and Innovation in the Information and Communication Technologies and Security Technologies Fields. Luxembourg: Publications Office of the European Union. 



\title{
Responsible Innovation: Neuer Ansatz der Gestaltung von Technik und Innovation oder nur ein Schlagwort?
}

\section{Armin Grunwald}

Institut für Technikfolgenabschätzung und Systemanalyse (ITAS), Karlsruhe

\section{Keywords}

Governance, Verantwortung, Technikgestaltung, Technikfolgenabschätzung, Constructive Technology Assessment

\begin{abstract}
Der Begriff der „Responsible Innovation“ ist ein relativ neues Schlagwort im Kontext der Gestaltung von Technik und Innovation, nach dem ethische Überlegungen, Folgenreflexion und die Beteiligung von Nutzern und Betroffenen den gesamten Prozess der Forschung und Technikentwicklung von den frühen Phasen im Labor über Entwurf, Design und Produktion bis hin zu marktreifen Innovationen begleiten sollen. In diesem Beitrag gehe ich der Frage nach, welche Ziele, Voraussetzungen und Implikationen diese Konzeptionen im Kontext der Technology Governance haben. Theoretische Untermauerungen oder theoriebasierte Beschreibungen gibt es bislang nicht. Die verfügbaren Texte entstammen vor allem dem forschungspolitischen und förderpolitischen Kontext. Daher geht es an dieser Stelle zunächst darum, den Gedanken der Responsible Innovation im Feld der bereits vorliegenden Ansätze der Technikgestaltung zu verorten.
\end{abstract}

\section{Responsible Innovation: A New Approach to Shaping Technology and Innovation or Just a Catchword?}

Keywords: Governance, responsibility, shaping technology, technology assessment

\begin{abstract}
Responsible Innovation' is a recently coined term in current debates on shaping technology and innovation. It requires that ethical considerations on impacts and consequences of new technology and innovation should accompany the entire process of research and development from early stages on, over the phases of design and production up to providing innovations for the marketplace. In doing this, users, stakeholders, and people possibly affected by impacts and consequences should be involved. Up to now neither a sound theoretical fundament nor a theory-based description are available. The term 'Responsible Innovation' roots in the fields of research policy and funding strategies, rather than in scientific approaches. Further clarification is needed. This paper addresses the question of the objectives, presuppositions, and implications of Responsible Innovation in the context of Technology Governance. The first step is to identify the relations between 'Responsible Innovation' and existing approaches to shaping technology.
\end{abstract}




\section{Einleitung}

In unterschiedlichen sozialkonstruktivistisch motivierten Konzeptionen der Technology Governance (z. B. SST - Social Shaping of Technology, Yoshinaka et al. 2003, oder CTA - Constructive Technology Assessment, Rip et al. 1995) werden Technikentwicklung und Innovationsgestaltung als soziale Prozesse verstanden, die mit politischen Implikationen, gesellschaftlichen Gruppen, ihren Strategien, Werten und Interessen verbunden sind. Dieses Verständnis eröffnet konzeptionell Möglichkeiten der gezielten Gestaltung im Hinblick auf normative Vorstellungen, z. B. im Hinblick auf nachhaltige oder verantwortliche Technik. Allerdings sind Möglichkeiten, Wege und Grenzen der Gestaltung und der Gestaltbarkeit von Technik umstritten. Pfadabhängigkeiten, Machtverhältnisse und Eigendynamiken, z. B. ökonomischer Art, schränken die Möglichkeiten der Gestaltung ein (Dolata/Werle 2007).

Da normativ der starke Wunsch besteht, die Grenzen der Gestaltbarkeit möglichst weit hinaus zu schieben und Gestaltungspotentiale so weit wie möglich auszuschöpfen, verwundert es nicht, dass meist in der Tradition des CTA immer neue Konzeptionen vorgeschlagen werden, in der Hoffnung, neue Gestaltungsmöglichkeiten zu eröffnen. Ein aktuelles Schlagwort in diesem Kontext ist das der „Responsible Innovation“, nach dem ethische Überlegungen, Folgenreflexion und die Beteiligung von Nutzern und Betroffenen den gesamten Prozess der Forschung und Technikentwicklung von den frühen Phasen im Labor über Entwurf, Design und Produktion bis hin zu marktreifen Innovationen begleiten sollen. Gelegentlich wird auch von „Responsible Development“ (Siune et al. 2009) oder von „Responsible Research and Innovation“ gesprochen.

In diesem Beitrag gehe ich der Frage nach, welche Ziele, Voraussetzungen und Implikationen diese Konzeptionen im Kontext der Technology Governance (Aichholzer et al. 2010) haben. Theoretische Untermauerungen oder theoriebasierte Beschreibungen gibt es bislang nicht. Die verfügbaren Texte sind kurz und rein programmatisch gehalten, sie entstammen vor allem dem forschungspolitischen und förderpolitischen Kontext, so beispielsweise im Forschungsprogramm „Responsible Innovation - Ethical and Societal Exploration of Science and Technology“ der niederländischen Forschungsförderung (Abschnitt 2), das eine der ersten Manifestationen dieses Ansatzes ist.

Da diese Konzepte aus dem politischen Kontext stammen, sowohl US-amerikanisch als auch europäisch, haben sie zunächst einen eher programmatischen, teils rhetorischen Charakter (Abschnitt 2). Es lässt sich jedoch zeigen, dass jenseits dieser forschungs- und innovationspolitischen Ebene Anknüpfungsmöglichkeiten an unterschiedliche Traditionen der Technikbewertung und Technikgestaltung bestehen (Abschnitt 4). Zusätzlich, und dies bringt die zentrale Stellung des Wortes ,responsible" mit sich, lassen sich verantwortungsethische Debatten anschließen, gleichwohl, und dies ist durchaus eine konzeptionelle Erweiterung bestehender Ansätze in diesem Bereich, muss dafür der Blick auf Verantwortungsfragen über die ethische Dimension hinaus erweitert werden. Verantwortungszuschreibung ist eine gesellschaftliche, teils politische, und nicht nur eine ethische Aufgabe. Ethik kann hier nur einen Teil der erforderlichen Leistungen einbringen, indem sie über die „Verantwortbarkeit“ und ihre Bedingungen reflektiert. Hinzukommen muss einerseits die Einbettung in die relevanten gesellschaftlichen Strukturen und die Governance des entsprechenden Innovations- und Technikfeldes, andererseits die Beachtung erkenntnistheoretischer Grenzen des Folgenwissens, da die Zuschreibung von Verantwortung an dessen Qualität gebunden ist (Abschnitt 3). 


\section{Responsible Innovation}

„Responsible Innovation“, gelegentlich auch „Responsible Development“ (Siune et al. 2009) oder „Responsible Research and Innovation“ sind Begriffe, die seit einigen Jahren mit zunehmender Resonanz kursieren. Zusammengehalten werden diese Begriffe durch das Attribut ,responsible“, was Anlass ist, in diesem Beitrag den Implikationen der starken Verwendung des Verantwortungsbegriffs in Bezug auf Technology Governance eigens nachzugehen (Abschnitt 3). Zunächst seien jedoch einige konkretere Manifestationen dieser Konzeptfamilie kurz vorgestellt.

Der Ursprung lässt sich nicht klar ausmachen. Frühe Erwähnungen sind jedenfalls forschungspolitischer bzw. förderpolitischer Art und stammen aus den USA, wo sie die National Nanotechnology Initiative (NNI) begleiten. Einerseits sagen sie etwas $\mathrm{zu}$ deren gesellschaftlicher Einbettung und Verpflichtung, wenn es z. B. heißt:

Responsible development of nanotechnology can be characterized as the balancing of efforts to maximize the technology's positive contributions and minimize its negative consequences. Thus, responsible development involves an examination both of applications and of potential implications. It implies a commitment to develop and use technology to help meet the most pressing human and societal needs, while making every reasonable effort to anticipate and mitigate adverse implications or unintended consequences.

(National Research Council 2006, S. 73)

Andererseits wird die Verpflichtung zur Verantwortung auch herangezogen, um die naturwissenschaftlich-technische Forschung, meist zur Nanotechnologie, nicht ihrer eigenen Dynamik zu überlassen, sondern sie entweder über ELSI-Forschung (ethical, legal and social implications) oder durch Selbstverpflichtungen und Ethik-Kodizes wenn auch nicht zu regulieren, sondern doch zu ,,begleiten“" und sie dadurch in Bezug auf gesellschaftliche Erwartungen und Folgen reflexiver zu gestalten (Grunwald 2011). In der Europäischen Union wurde 2008 ein Code of Conduct für die Nanotechnologie verabschiedet, der ebenfalls auf dieser Linie liegt. Dies gilt auch für die Ausrichtung der britischen Forschungsförderung in diesem Bereich, primär bezogen auf die Identifizierung möglicher Risiken und den verantwortlichen Umgang mit ihnen:

Through its Nanoscience Grand Challenge call in 2009, EPSRC ${ }^{1}$ included for the first time a requirement for applicants to include their plans for „Responsible Innovation”. Through this they were asked to consider the wider implications of their proposed research (on society, the environment and health) and qualitatively assess the level of risk and uncertainty associated with potential impacts. Applicants were asked to submit a 'risk register' which was subsequently peer reviewed for eventual consideration by the funding panel. The objective has been to equip both the applicants and research councils with tools to identify and manage wider risks associated with innovation in an upstream, proactive and proportionate manner, building on recommendations made by the Royal Commission on Environmental Pollution in their 2008 Novel Materials report and the Royal Society and Royal Academy of Engineering in their 2004 Nanotechnology report.

(see www.epsrc.ac.uk/funding/calls/2010/Pages/responsibleinnovation.aspx)

Seit dem Jahr 2009 besteht in den Niederlanden das Programm „Responsible Innovation - Ethical and Societal Exploration of Science and Technology“ (abgekürzt MVI nach der niederländischen Fassung). Ausgerichtet von der „Dutch Organisation for Scientific Research“ (NWO), Pendant der

1 EPSCR - Engineering and Physical Sciences Research Council - is the main UK government agency for funding research and training in engineering and the physical sciences (see www.epsrc.ac.uk). 
Deutschen Forschungsgemeinschaft, und finanziert durch Mittel aus vier Ministerien, dient es der Förderung von Projekten, in denen die Erforschung ethischer und sozialer Fragen neuer Technologien in einen engen Kontext mit Technikentwicklung gestellt wird. In der Selbstbeschreibung lautet dies:

The MVI program focuses on technological developments for which we can expect that they will have an impact on society. On the one hand, those developments concern new technologies (such as ICT, nanotechnology, biotechnology and cognitive neuroscience), and on the other, technological systems in transition (for example agriculture and healthcare). The MVI contributes to responsible innovation by increasing the scope and depth of research into societal and ethical aspects of science and technology

(www.nwo.nl/nwohome.nsf/pages/nwoa_7e2ezg_eng).

Charakteristisch ist, dass das Programm nicht auf eine bloße Verbesserung des Verständnisses von sozialen und ethischen Fragen in Wissenschaft und Technik zielt, sondern dass vielmehr eine „Make“-Perspektive eingenommen wird. Das Programm will letztlich durch die Erforschung von ethischen und gesellschaftlichen Aspekten von Technik möglichst direkt zu ihrer adäquaten Gestaltung beitragen:

Projects for research into ethical and societal aspects of concrete technological developments must always have a 'makeable' perspective. In other words, they must not only lead to an analysis and an improved understanding of problems, but also result in a 'design perspective' - in the broadest sense, including institutional arrangements

(www.nwo.nl/nwohome.nsf/pages/nwoa_7e2ezg_eng).

Gemäß diesem Anspruch wurde die Auswahl der geförderten Projekte nicht nur unter wissenschaftlichen Kriterien vorgenommen, sondern es war ein „Societal Panel“ bestehend aus Stakeholdern, das die Plausibilität der Erreichung dieses Anspruches auf gesellschaftliche Wirksamkeit prüfte. Dieses Panel hatte eine starke Stellung, da es Projekte aus Gründen mangelnder gesellschaftlicher Relevanz aussortieren konnte, ohne dass die wissenschaftliche Prüfung ein solches Urteil hätte revidieren können. Die kritische Begleitung der Umsetzung der versprochenen gesellschaftlichen Relevanz ist in allen Projekten einem begleitenden „Valorisation Panel“ überantwortet, bestehend im Wesentlichen aus Stakeholdern aus dem betroffenen Bereich. Natürlich werden auch Publikationen erwartet - die wesentliche und sich von anderen Programmen unterscheidende Anforderung ist aber die, konkrete Beiträge zu Gestaltung und Problemlösung zu erbringen.

Nach dem Start Ende 2009 mit etwa 15 Projekten aus unterschiedlichen Themenfeldern (vgl. www.nwo.nl/nwohome.nsf/pages/nwoa_7e2ezg_eng) kamen Ende 2010 in einer zweiten Ausschreibungsrunde weitere Projekte hinzu. Im April 2011 fand in Den Haag eine erste Konferenz dieses Programms statt. Auf dieser konnten sich einerseits diese Projekte in einem internationalen Rahmen vorstellen, andererseits waren aber auch externe Vorträge eingeladen, die sich dem Grundgedanken von „Responsible Innovation“ verwandt fühlen.

Zwei Beispiele für Projekte seien kurz erwähnt:

Im Projekt „New economic dynamics in small producers clusters in northern Vietnam - Institutions and responsible innovation with regard to poverty alleviation " werden Wertschöpfungsketten lokaler Hersteller untersucht. Das Projekt „,builds further on the research outcome by exploring the potential importance of these specific technological cases for poverty reduction in developing countries, thus whether the innovations could be labelled as 'responsible innovations'. Vietnam offers a particularly interesting research context since the innovations of poor small producers are based on private initiatives with an institutional environment in transition" (ebd.). Mit diesem Projekt werden folgende Ziele verfolgt (ebd.): 
- "to understand the concept of 'responsible innovation' and its valorization in small producers' clusters in northern Vietnam,

- to explain the multi-level institutional framework enabling and facilitating the small producers to innovate, and

- to assess how the institutional framework interacts with small producers' economic behaviour through incentives."

Auf diese Weise sollen konkrete Anregungen entwickelt werden, wie fragile Wertschöpfungsketten unter den dortigen Bedingungen stabilisiert und gestärkt werden können. Das Projekt „, Matching supply and demand side preferences in food innovation " ist auf Übergewichtigkeit und Ernährungsgewohnheiten fokussiert. Die Problematik wird als ein Henne/Ei-Problem ohne klare Kausalität beschrieben: weder sei die Angebotsseite allein verantwortlich zu machen noch die Nachfrageseite. Vielmehr gebe es zwischen beiden eine gegenseitige Abhängigkeit. Mögliche Veränderungen in diesem System werden mit einem Innovationssystemansatz erforscht:

The project takes an innovation systems approach, which emphasizes the interdependencies between actors and actions in the innovation process. Given actor preferences, it aims to create conditions under which both supply and demand have the right incentives to act in a societal responsible manner. This form of structural mapping and matching of actor preferences is a novel approach in innovation systems thinking. Using conjoint analyses, the preferences of the demand and supply side will be identified and market segments will be identified. Further, conditions will be sought under which consumers prefer to consume healthier food and suppliers prefer to produce healthier products (ebd.).

Das Ziel ist letztlich, durch das entstehende Wissen zu Veränderungen in der Realwelt beizutragen. In beiden Fällen wacht das bereits erwähnte „Valorisation Panel“ darüber, dass sich die wissenschaftliche Eigendynamik, die letztlich ausschließlich nach Kriterien des Wissenschaftssystems operiert und die Belange der Praxis zu vernachlässigen droht, nicht durchsetzt.

Der konkrete Charakter dieser (und auch der anderen, hier nicht erwähnten) Projekte täuscht nicht darüber hinweg, dass „Responsible Innovation“ bislang nicht viel mehr als ein forschungspolitisches Schlagwort zu sein scheint. Auch in anderen Konzeptionen von Technikethik (z. B. das Value Sensitive Design, van de Poel 2009) und Technikfolgenabschätzung (Grunwald 2010) hätten ähnliche Projekte ihren Platz gefunden. Zum forschungspolitischen Charakter passt auch, dass das achte Forschungsrahmenprogramm der Europäischen Kommission, das stark auf Innovation setzt, voraussichtlich einen Teil enthalten wird, der der „Responsible Innovation“ gewidmet ist.

Aber auch wenn die These vom Schlagwortcharakter zutreffen sollte, ist eine weitergehende Befassung angebracht, und zwar zumindest aus drei Gründen:

- Selbst wenn es hinter dem Schlagwort „Responsible Innovation“ keine konzeptionelle Substanz gäbe, würde es die Praxis der Forschungs- und Technikförderung alleine durch seine Existenz beeinflussen. Begutachtungsverfahren und Qualitätskriterien sind jedenfalls, wenn man das NWOProgramm als Vorbild nimmt, teils andere als vor der Anerkennung dieses Schlagworts. Damit hat „Responsible Innovation“ bereits deshalb Auswirkungen in der Technology Governance, bloß weil es existiert.

- Diese praktischen Veränderungen wären in Bezug auf ihre theoretischen Voraussetzungen, ihre Wurzeln in älteren Konzepten und ihre praktischen Folgen hin zu befragen, um die Steuerungswirkungen von „Responsible Innovation“ möglichst transparent aufzudecken. Schließlich kann dafür argumentiert werden, dass „Responsible Innovation“ sich aus Quellen aus der Technikfolgenabschätzung, der Technikethik und der Governance-Forschung speist (Abschnitt 4).

- Dieses sollten Theoretiker und Praktiker aus den Feldern der Technikfolgenabschätzung und Technikethik zum Anlass nehmen, eigenständig an einem substantiellen Konzept des „Respon- 
sible Innovation“ zu arbeiten und daher dazu beizutragen, dass die inhaltliche Leerstelle, die mit einem bloßen Schlagwort verbunden wäre, allmählich durch konzeptionelle Arbeit und mit Inhalten gefüllt wird.

Dies ist Anlass, um im Folgenden zum einen dem zentralen Element von „Responsible Innovation“, dem Verantwortungsbegriff und seiner Rolle in der Technology Governance (Abschnitt 3) nachzugehen. Zum anderen wird nach ihren konzeptionellen Quellen gefragt (Abschnitt 4).

\section{Dimensionen der Verantwortung}

Eine begriffliche oder konzeptionelle Klärung des Verantwortungsbegriffs findet sich in den (bislang wenigen) Texten zur Responsible Innovation nicht. Vielmehr wird an ein intuitives Verständnis appelliert, häufig in Zusammenhang mit Risikobefürchtungen und nicht intendierten gesellschaftlichen Folgen von Innovationen. Das Wort „verantwortlich“ wird als Chiffre verwendet, um einen (vermeintlich klaren) Weg zu bezeichnen, mit der bekannten Ambivalenz von Technik und Innovation (Grunwald 2010, Kap. 1) und den nicht intendierten Folgen von Innovation umzugehen. Die Lösung erscheint, begrifflich gesehen, einfach: Man solle einfach ,verantwortlich“ mit diesen negativen Begleiterscheinungen des Fortschritts umgehen.

Insofern dies so daherkommt, ist Verantwortung wirklich nicht mehr als ein rhetorisches Schlagwort. Die damit womöglich verbundene Hoffnung zerschellt allerdings bereits an der vielfachen Begriffskritik, geradezu der Häme, die gegenüber Verantwortung und Verantwortungsethik vor allem seitens der Sozialwissenschaften vorgebracht wird. Verantwortung sei inhaltsleer, eine bloße Phrase mit nicht mehr als hilflosem Appellcharakter, mehr ein Moralisieren des Typs ,man müsste doch Verantwortung übernehmen“ als valide Problembeschreibung und Vorschlag möglicher Problemlösungen, ein bloßes Moralisieren in Bezug auf die Verantwortungslosigkeit in Verbindung mit Wunschdenken in Bezug auf aktive Verantwortungsübernahme. Auch werden Diagnosen vorgebracht, dass komplexe Verursachungsgefüge und Akteurskonstellationen in der modernen Wissenschaft, Technikentwicklung und Innovation zwangsläufig dazu führen müssten, dass nicht mehr einzelne Akteure verantwortlich gemacht werden könnten, sondern es zu einer ,Verantwortungsverdünnung" komme, so dass Verantwortung nur noch in homöopathischer Dosis beim Einzelnen hängen bleibe und es keinerlei Möglichkeit mehr gebe, diese verteilte Verantwortung zu irgend einer aussichtsreichen Intervention zu nutzen. Ein Klassiker ist hier die Frage nach der Verantwortung der Autofahrer für den Klimawandel. Auch die Unsicherheiten des Wissens über die Folgen des Handelns werden als Argument gegen die Möglichkeit von Verantwortungsethik ins Feld geführt mit zunehmender Unsicherheit verschwinde der Gegenstand der Verantwortung (Bechmann 1993). Ulrich Beck, einer der rhetorisch schärfsten Kritiker, hat sogar eine Gegenwartsdiagnose in Buchform mit „Die organisierte Unverantwortlichkeit“ betitelt (Beck 1988). Dies alles vor Augen müssen dann in der Tat Bekundungen des folgenden Typs weltfremd oder verdächtig erscheinen:

"Good governance of N\&N research should take into account the need and desire of all stakeholders to be aware of the specific challenges and opportunities raised by N\&N. A general culture of responsibility should be created in view of challenges and opportunities that may be raised in the future and that we cannot at present foresee." (EC 2008)

An dieser Stelle scheiden sich die Geister. Die einen würden bei der obigen Diagnose bleiben und vielleicht eine Ideologiekritik der Verantwortung anschließen, aber ansonsten dazu raten, sich wissenschaftlich von diesem Begriff fernzuhalten. Die anderen - zu denen gehört der Autor dieser Zeilen - könnten sagen, dass man diesen Schritt nicht voreilig tun solle. Denn die genannten Kri- 
tikpunkte am Verantwortungsbegriff sind ja nicht unwidersprochen geblieben (z. B. Grunwald 1999) und haben auch nicht dazu geführt, dass der Verantwortungsbegriff gemieden wird. Vielleicht haben ja die Kritiker nicht Recht, zumindest nicht in allen Facetten. Anerkennend, dass die Kritiker vielfach zu Recht auf ernstzunehmende Probleme des Verantwortungsbegriffs aufmerksam machen, werde ich im Folgenden eine kurze begriffliche Analyse unternehmen, um damit die These zu entfalten und zu begründen, dass der Verantwortungsbegriff, soll er den genannten Kritikpunkten widerstehen, spezifischer und genauer gefasst werden muss als dies üblicherweise in der Verantwortungsethik erfolgt.

Verantwortung ist Resultat einer Zuschreibungshandlung, entweder wenn Handelnde sie sich selbst zuschreiben und damit etwas über die Beurteilung ihrer eigenen Handlungen oder den Umgang mit deren Folgen aussagen oder durch die Verantwortungszuschreibung durch andere (Grunwald 1999). Die Rede „Wer trägt welche Verantwortung?“ ist verkürzt: Die Zuschreibung von Verantwortung stellt selbst eine Handlung dar, welche unter Zwecken und relativ zu Zuschreibungsregeln erfolgt. Diese Zuschreibungsregeln sind selbst rechtfertigungspflichtig, indem sie z. B. den Kreis der verantwortungsfähigen Individuen abgrenzen und Kriterien angeben, welche Voraussetzungen Individuen erfüllen müssen, um zur Verantwortung gezogen werden zu können. Auf diese Weise steht Verantwortung von Beginn an in sozialen Bezügen der Zuschreibung und Verpflichtung bzw. entsprechender Erwartungen. Diese untrennbare und konstitutive Verortung des Verantwortungsbegriffs in sozialen Zusammenhängen ist eines der Elemente, das in verantwortungsethischen Überlegungen teils fehlt oder zu kurz kommt, was dann sofort den sozialwissenschaftlichen Reflex auslöst, Verantwortungsdebatten als abgelöst von realen Entwicklungen und damit als irrelevant für die Governance anzusehen, eben als bloß rhetorisch nutzbares Schlagwort.

Die Fassung des Verantwortungsbegriffs als Zuschreibungsbegriff hat Folgen. Die Forderung nach pragmatischer Konsistenz rationaler Akteure führt dazu, dass eine Instanz, die bestimmten Personen oder Gruppen eine spezifische Verantwortung zuschreibt, darauf achten muss, ob diese die zugeschriebene Verantwortung auch faktisch wahrnehmen können. Wenn nicht, würde die Zuschreibung ins Leere laufen und bliebe belanglos, ohne dass den Akteuren, denen die Verantwortung zugeschrieben worden war, daraus ein Vorwurf gemacht werden könnte. Wenn die zuschreibende Instanz nun selbst in der Lage ist, die Bedingungen zu beeinflussen, unter denen die Übernahme von Verantwortung praktisch möglich ist, kann dieses Argument der pragmatischen Konsistenz dahingehend verschärft werden, dass die zuschreibende Instanz sich damit selbst verpflichtet, die Bedingungen zu schaffen, unter denen die verantwortlich gemachten Akteure ihrer Verantwortung auch gerecht werden können. Die Verpflichtung Anderer geht einher mit der Selbstverpflichtung, adäquate Bedingungen zu schaffen. Dieses kann ein starkes Argument sein, vor allem, wenn die Verantwortung abstrakt von 'der Gesellschaft' zugeschrieben wird.

Der prospektive Verantwortungsbegriff als Verantwortung für zukünftige, noch nicht eingetretene Handlungs- und Entscheidungsfolgen (der retrospektive interessiert in diesem Beitrag nicht) muss semantisch als zumindest dreistelliger Begriff rekonstruiert werden: Jemand (ein Verantwortungssubjekt) verantwortet etwas (Handlungsresultate als Objekt der Verantwortung) vor einer Instanz (z. B. einer Person oder einer Institution). Die moralische Dimension erschließt sich erst in einer Rekonstruktion des Verantwortungsbegriffs als vierstellig, wenn nämlich gefragt wird, relativ zu welchen Regelsystemen, Werten oder Normen Verantwortung übernommen werden soll. Diese Regeln bilden den normativen Rahmen für die Beurteilung von Handlungen als verantwortbar. Kommt es hier zu normativen Unsicherheiten oder zu moralischen Konflikten, ist eine ethische Reflexion auf diese Regeln und ihre Rechtfertigbarkeit erforderlich.

Für viele Zwecke, vor allem in Bezug auf Verantwortung für Wissenschaft und Technik, ist darüber hinaus eine fünfstellige Rekonstruktion angemessen (Grunwald 2011): (1) Jemand ist verantwortlich (2) für etwas (3) vor einer Instanz (4) relativ zu einem Regelwerk und (5) relativ zu einem Wissensstand. Der Bezug auf einen Stand verfügbaren Wissens ist in Fragen prospektiver Verant- 
wortung unverzichtbar. In Verantwortungsdebatten muss der Stand des verfügbaren Wissens über die zu verantwortende Zukunft erhoben und unter epistemologischen Aspekten, d. h. in Bezug zur Qualität und Belastbarkeit des Folgenwissens, kritisch reflektiert werden. In Bezug auf weit reichende Zukunftsfragen, die nur unter hoher Unsicherheit beurteilt werden können, ist dies ein entscheidender Aspekt, indem der Relation zwischen dem verfügbaren Wissen - dem ,was auf dem Spiel“" steht - und den normativen Kriterien für Verantwortung und Verantwortbarkeit die entscheidende Bedeutung zukommt. Ein schönes Zitat in diesem Kontext stammt aus der Synthetischen Biologie:

Fifty years from now, synthetic biology will be as pervasive and transformative as is electronics today. And as with that technology, the applications and impacts are impossible to predict in the field's nascent stages. Nevertheless, the decisions we make now will have enormous impact on the shape of this future (Ilulissat Statement 2007, S. 2).

Wenn dies zutrifft, haben die heutigen Entscheidungen einen weit reichenden, wenn nicht dominierenden Einfluss auf die zukünftigen Entwicklungen - aber wir wissen nicht, welchen. Wenn dies das letzte Wort wäre, würde jede Verantwortungsethik obsolet: Das, was nicht gewusst werden kann, kann auch nicht verantwortet werden. Mit dem Wissen über mögliche Entscheidungsfolgen verschwindet auch der Gegenstand einer Verantwortungsethik. Ist also, wie eingangs gesagt, die normative Verantwortungsdebatte in Kontakt mit den empirischen gesellschaftlichen Verhältnissen zu bringen, müssen „nach der anderen Seite“ epistemische Bedingungen der Möglichkeit von Verantwortung bedacht werden. Damit stellen sich in prospektiven Verantwortungsdebatten Fragen in drei Dimensionen (Grunwald 2011):

- Governance-Dimension: Wie soll Verantwortung verteilt werden, welche gesellschaftlichen Gruppen sind betroffen und sollen über die Verantwortungsverteilung mitentscheiden. Handelt es sich um Fragen, die die „Polis“ betreffen oder können sie an Gruppen oder Teilsysteme delegiert werden? Diese Dimension erstreckt sich auf die Subjekte, denen Verantwortung zugeschrieben wird, und auf ihr Verhältnis zu den Objekten und der Instanz, vor der dieses geschieht. Die Governance-Dimension fasst somit die ersten drei des oben genannten fünfstelligen Verantwortungsbegriffs zusammen und betont ihren sozialen Kontext.

- ethische Dimension: Können die in Frage stehenden Handlungen, z. B. wissenschaftliche Entwicklungen, gerechtfertigt werden, unter welchen Bedingungen können sie dies? Welche ethischen Reflexions- und Argumentationsmuster sind einschlägig?

- epistemische Dimension: Was wird gewusst, was kann gewusst werden, welche Unsicherheiten bestehen fort, wie können sie qualifiziert werden und was steht im Falle des Falles auf dem Spiel?

Häufig werden Verantwortungsdebatten auf die ethische Dimension beschränkt und ausschließlich im Rahmen der Verantwortungsethik behandelt. Resultat sind die bekannten und teils oben genannten Vorwürfe des bloß Appellativen, der epistemologischen Blindheit und der Naivität in Bezug auf soziale Kontexte. Wenn man dagegen die hier angedeutete semantische Diversität des Verantwortungsbegriffs ernst nimmt, erscheint es zumindest möglich, den erwähnten Verdikten zu entgehen und den Begriff für gehaltvolle Analysen und für Governance-Fragen fruchtbar zu machen.

Durch die bisherigen Überlegungen hat sich zweierlei klar gezeigt. Zum einen dürfen Fragen der Zuschreibung von Verantwortung generell nicht individualistisch verengt gestellt und beantwortet werden. Stattdessen bedarf es der Einbettung in die umgebenden gesellschaftlichen Verhältnisse, der Beachtung der Governance-Strukturen und der Rücksicht auf Mitspracherechte und demokratische Beteiligungsansprüche, insofern Fragen betroffen sind, die die Polis betreffen. Zum anderen muss in Verantwortungszuschreibungen auf die Wissensstrukturen, Wissensprobleme und Kompetenzen der Beteiligten Rücksicht genommen werden. Verantwortungszuschreibung stellt sich auf diese Weise als komplexes, die Reichweite philosophischer Ethik weit übersteigendes Unterfangen heraus. 
Bestimmte, besonders in der öffentlichen, aber auch teils in der ethischen Debatte verbreitete Strukturen der Verantwortungszuschreibung erscheinen vor diesem Hintergrund unterkomplex, wenn nicht gar naiv. Oft wird gefordert, dass Wissenschaftler die Folgen ihres Handelns in der Weise einer kompletten Wissenschafts- und Technikfolgenbeurteilung reflektieren sollen, verbunden mit der impliziten Hoffnung, dass wenn Wissenschaftler umfassend die Folge des eigenen Handelns einschätzen, diese verantwortlich beurteilen und entsprechend handeln würden, negative, nicht intendierte Folgen weitgehend oder komplett vermieden werden könnten. Diese Erwartungen scheitern an den beiden genannten Aspekten: Sie werden der Einbettung wissenschaftlichen Handelns in eine demokratische Gesellschaft nicht gerecht und verkennen die Wissens- und Beurteilungsprobleme. Weder individuelle Wissenschaftler noch Disziplinen können allein diese Fragen Erfolg versprechend bearbeiten. Synthetische Biologen beispielsweise sind Experten für Synthetische Biologie, nicht für mögliche gesellschaftliche Folgen ihres Handelns und auch nicht für die Frage der Akzeptabilität unklarer Risiken und den Umgang mit ihnen. Vor einem unreflektierten Verantwortungsoptimismus ist daher zu warnen, sowohl wenn Wissenschaftler und Ingenieure sich selbst unter diese Erwartungen stellen als auch wenn sie seitens der Gesellschaft darauf verpflichtet werden sollen. „Responsible Innovation“ verweist mit Recht darauf, dass über Verantwortung für Wissenschaft, Technik und Innovation sinnvoll nur interdisziplinär gesprochen werden kann.

Verantwortung in Fragen des wissenschaftlich-technischen Fortschritts und seiner Umsetzung in Innovation kann nur arbeitsteilig getragen werden. Die Technikwissenschaft, um die es im Einzelfall geht, z. B. die Synthetische Biologie (Grunwald 2011) hat in diesem Konzert der Verantwortungsträger einen besonderen Platz, weil ihr Wissen durch andere Beteiligte nicht ersetzt werden kann. Sie spielt jedoch nur ein Instrument unter vielen. Andere Beteiligte sind zum einen weitere Wissenschaften wie die Governance-Forschung, die Wissenschaftsforschung, Ethik und Technikfolgenabschätzung, Risikoforschung und, sobald die Entwicklung soweit ist, auch Innovationsforschung. Das Konzert der Verantwortungsträger ist damit zunächst ein interdisziplinäres Konzert.

Die involvierte demokratische Dimension führt dazu, dass dieses Konzert darüber hinaus auch transdisziplinär sein muss. Beteiligungsansprüche von Bürgern und Initiativen von Verbänden und Nichtregierungsorganisationen regen die gesellschaftliche Deliberation an. Medien transportieren diese Debatten in eine größere Öffentlichkeit. Akademien und Stiftungen, aber auch politische Institutionen sollten diese Deliberation unterstützen. Die Debatte zur Verantwortung der Wissenschaft darf vor dem geschilderten Hintergrund die pragmatistische Dimension (Habermas 1968) nicht außer Acht lassen und sich nicht auf enge Expertenzirkel beschränken.

\section{Vorläufer der „Responsible Innovation“}

Das Konzept des Responsible Innovation lässt sich als Zusammenführung verschiedener Traditionen der Technikreflexion und Technikgestaltung verstehen (Grunwald 2012). ${ }^{2}$ Die im Folgenden genannten Ansätze haben je durch verschiedene Akzente zum Konzept der „Responsible Innovation" beigetragen. In der Darstellung wird kein Anspruch auf Vollständigkeit erhoben; so könnten z. B. auch Ansätze wie die innovationsorientierte Technikfolgenabschätzung (Steinmüller et al. 1999) genannt werden, die die Verschiebung von „Technik“ zu „Innovation“ begleitet und verstärkt haben, ohne den die „Responsible Innovation“ ebenfalls kaum denkbar wäre. Eine vollständige Genealogie der Responsible Innovation kann jedoch an dieser Stelle nicht geleistet werden.

2 Die Texte in diesem Abschnitt sind Überarbeitungen der entsprechenden Beschreibungen aus Grunwald (2010). 


\section{I Constructive Technology Assessment}

Das (CTA) ist in den Niederlanden entwickelt, aber auch in anderen europäischen Ländern diskutiert und praktiziert worden (Rip et al. 1995). CTA beruht auf der Vorstellung, dass die Entwicklung einer Technologie ein nahtloses Gewebe ('seamless web') von hochgradig heterogenen sozialen, kulturellen, ökonomischen, technischen und naturwissenschaftlichen Faktoren darstellt, in dem permanent Weichenstellungen stattfinden (Schwarz 1992). Eine ebenfalls permanente Technikfolgenabschätzung solle diesen quasi-naturwüchsigen Prozess begleiten, informieren, reflektieren und dadurch bewusster gestalten. Ziel der CTA ist es, ,ein Bild des sozialen Prozesses innerhalb der Technikentwicklung [zu bilden], das prinzipiell zahlreiche Möglichkeiten und die richtigen Zeitpunkte anzeigte, um auf der Basis gesellschaftlicher Ziele Einfluss nehmen zu können“ (Boxsel 1991, S. 143). In diesem Sinne ist CTA vor allem als ,aktives Management der Prozesse des technologischen Wandels“ (Schot 1992, S. 36) zu verstehen; das Ziel ist es ,to achieve better technology in a better society“" (Schot/Rip 1997).

Der theoretische Hintergrund dieses Konzeptes liegt in der sozialkonstruktivistischen Technikforschung begründet, wie sie ebenfalls in den Niederlanden entwickelt und im SCOT-Programm (Social Construction of Technology) in einer Fülle von Fallstudien geprüft wurde (Bijker et al. 1987; Rip et al. 1995). In genauer Entgegensetzung zu jedweder Form eines technologischen Determinismus wird hier die Technikentwicklung als Resultat gesellschaftlicher Meinungsbildungs- und Aushandlungsprozesse sowie von Entscheidungen verstanden. Technik werde durch diese Schritte gesellschaftlich ,konstruiert“. Dies äußert sich in prominenten Buchtiteln wie „Shaping Technology - Building Society“ (Bijker/Law 1994) oder „Managing Technology in Society“ (Rip et al. 1995). Aufgrund der Probleme mit einer staatlichen Techniksteuerung hat sich CTA früh für eine breite Einbeziehung von gesellschaftlichen Akteuren, insbesondere auch der Wirtschaft, und für die Ausprägung einer lernenden und mit Technik experimentierenden Gesellschaft ausgesprochen. Responsible Innovation lässt sich als direkte Weiterführung dieses Ansatzes unter Betonung von Verantwortungsfragen verstehen.

\subsection{Der Ansatz der Technikbewertung des VDI}

Die VDI-Richtlinie 3780 wurde 1991 publiziert und gehört zu den am weitesten verbreiteten Dokumenten der Technikfolgenabschätzung (VDI 1991). Technik im Sinne der Richtlinie bezieht sich nicht nur auf Technik im Sinne dinghafter Artefakte, sondern auch auf die damit verbundenen menschlichen Handlungskontexte der Technikentstehung (Forschung, Entwurf, Entwicklung, Produktion), der Techniknutzung und der Entsorgung nutzlos gewordener Technik. Unter Technikbewertung wird ,das planmäßige, systematische, organisierte Vorgehen verstanden,

- das den Stand einer Technik und ihre Entwicklungsmöglichkeiten analysiert,

- unmittelbare und mittelbare technische, wirtschaftliche, gesundheitliche, ökologische, humane, soziale und andere Folgen dieser Technik und möglicher Alternativen abschätzt,

- aufgrund definierter Ziele und Werte diese Folgen beurteilt oder auch weitere wünschenswerte Entwicklungen fordert,

- Handlungs- und Gestaltungsmöglichkeiten daraus herleitet und ausarbeitet“ (nach Rapp 1999, S. 222f.).

Angesichts der Tatsache, dass Technikbewertung von Ingenieuren und in der Industrie immer betrieben wird, wenn z. B. eine Techniklinie als aussichtsreich, eine andere als Sackgasse bewertet wird, wenn zukünftige Produktchancen bewertet werden oder ein neues Produktionsverfahren im 
Betrieb eingeführt werden soll, sieht der VDI das Neue an dieser Richtlinie zum einen in der Breite des Bewertungshorizontes, in dem über technische und wirtschaftliche Faktoren hinaus weitere Folgendimensionen zu berücksichtigen sind, und zum anderen in der gesellschaftlichen Organisation der Bewertungsprozesse, die netzwerkartig über die engeren Bereiche der Ingenieure und des Managements hinaus gehen sollen. Dies sieht der VDI nur im Rahmen eines die Technikentwicklung ständig begleitenden Prozesses als möglich an.

Die Bewertung soll im VDI-Modell nach gesellschaftlich anerkannten Werten erfolgen. Acht zentrale Werte wurden identifiziert, die das mittlerweile bekannte „Werte-Oktogon“ des VDI bilden: Funktionsfähigkeit, Wirtschaftlichkeit, Wohlstand, Sicherheit, Gesundheit, Umweltqualität, Persönlichkeitsentfaltung und Gesellschaftsqualität. Diese Werte sollen das technische Handeln prägen und stehen unter der Prämisse (VDI 1991, S. 7): „Das Ziel allen technischen Handelns soll es sein, die menschlichen Lebensmöglichkeiten [...] zu sichern und zu verbessern“. Den Anschluss an die Technikentwicklung gewinnen sie, indem sie von den Ingenieuren in ihrer Praxis beachtet, d. h. in die Technik quasi eingebaut werden. Ingenieure bzw. Wissenschaftler sollen aufgrund ihres Wissens und Könnens die Technikentwicklung durch die Beachtung dieser Werte in die 'richtige' Richtung lenken und Fehlentwicklungen vermeiden. Technik soll so entwickelt werden, dass sie in Einklang mit diesen Werten steht. Der Zusammenhang mit „Responsible Innovation“ ergibt sich aus genau dieser hier erhobenen Forderung. Die Werte des Werte-Oktogons lassen sich als Ausdruck der Richtungen verstehen, in denen es in der Technikgestaltung durch Ingenieure um gesellschaftliche Verantwortung geht. Damit wird hier auch die Verbindung zur Ingenieursethik deutlich (z. B. Ropohl 1996).

\subsection{Verantwortungsethik}

Die Frage nach der Verantwortung von Wissenschaftlern und ihren Grenzen füllt spätestens seit der Erfindung der Atombombe Bücher, Tagungsprogramme und Leserbriefspalten in Zeitungen (z. B. Durbin/Lenk 1987). Normativer Ausgangspunkt für Beurteilungen der Folgen des wissenschaftlich-technischen Fortschritts war angesichts einer Vielzahl globaler Schreckensszenarien mit der Möglichkeit eines Endes der Menschheitsgeschichte zunächst die „unbedingte Pflicht der Menschheit zum Dasein“ (Jonas): „Niemals darf Existenz oder Wesen des Menschen im Ganzen zum Einsatz [...] gemacht werden“ (Jonas 1979, S. 81). Eine „Heuristik der Furcht“ in Kombination mit dem Prinzip des „Vorrangs der schlechten Prognose“ soll Orientierungen ermöglichen, wie mit technischen Innovationen umzugehen sei. Es resultiert nach Jonas ein kategorischer Imperativ, so zu handeln, dass ,die Wirkungen deiner Handlungen verträglich sind mit der Permanenz echten menschlichen Lebens auf der Erde“ (ebd., S. 36).

Neuere Formulierungen stellen das Adressatenproblem der Verantwortung in den Mittelpunkt: Angesichts komplexer arbeitsteiliger Handlungszusammenhänge gehe es darum, das Subjekt der Verantwortung zu bestimmen, um zu verhindern, dass Verantwortungsethik zu einem adressatenlosen Moralisieren werde und es zu einer „Verantwortungsverdünnung“ komme (Bechmann 1993). Die Arbeitsteiligkeit des Handelns dürfe die Folgenverantwortung nicht einfach auflösen, sondern es gehe darum, sie auf die involvierten Individuen nach Maßgabe ihrer Bedeutung und Handlungsmöglichkeiten in dem betreffenden kollektiven Handlungszusammenhang zu verteilen und institutionelle Möglichkeiten zu schaffen, diese verteilte Verantwortung auch zusammenführen zu können (Lenk 1992). Die Diskussionen der Verantwortungsethik können, zumindest in Bezug auf die ethische, weniger mit Bezug auf die anderen Dimensionen der Verantwortung (Abschnitt 3), als unmittelbare Vorbereitung für Responsible Innovation verstanden werden. 


\section{Schluss}

Ohne Zweifel, das hat der vorherige Abschnitt zumindest exemplarisch gezeigt, gibt es eine Reihe von Vorläufertraditionen des Responsible Innovation. Anliegen unterschiedlicher Konzeptionen der Technikfolgenabschätzung und der Technikethik werden aufgenommen und mit Nuancen weitergeführt. Das Element des radikal Neuen eignet der Responsible Innovation sicher nicht. Genauso kann gesagt werden, dass der „Responsible Innovation“ und ihren Verwandten der Anschein des bloß Rhetorischen anhaftet, der Verdacht, dass es mehr um politische Programmatik und Außenwirkung gehe als um substantielle Veränderungen der Technology Governance, die letztlich zu einer besser verantwortbaren Technik und Innovation führen könnten.

Beide Einwände könnte man zum Anlass nehmen, über Responsible Innovation wie über eine bloße Modewelle hinwegzugehen. Im vorliegenden Beitrag habe ich versucht, genau das Gegenteil zu tun: Den Begriff zunächst ernst zu nehmen und offen zu schauen, ob hier nicht ein semantisches und konzeptionelles Potential enthalten ist, dem es lohnt nachzuspüren, um allen Gestaltungspotentialen in Richtung Verantwortung nachzugehen und nichts ungeprüft zu lassen oder voreilig auszusortieren. Der Weg hat gezeigt, dass die Semantik des Verantwortungsbegriffs, insbesondere seine Auffächerung auf die ethische, die epistemologische und die Governance-Dimension es erlaubt, hier durchaus Hoffnungen in Bezug auf ein klareres Verständnis der Technology Governance und damit möglicherweise auch zu ihrer gezielten Beeinflussung zu gelangen.

Eine gewisse Parallele zwischen den Begriffsproblemen der „Nachhaltigkeit“ und der „Responsible Innovation“" sei genutzt, um die Motivation zu erhöhen, sich in dieser Richtung weiter konzeptionell und durch Forschung zu engagieren. Beide Begriffe teilen die Eigenschaft, dass man nicht konsistent dagegen argumentieren kann. Weder für eine explizit nicht nachhaltige Entwicklung noch für eine explizit nicht verantwortbare Innovation lässt sich argumentieren. Und beide Begriffe sind dem Vorwurf der Inhaltsleere und der bloßen Rhetorik ausgeliefert. Was wäre geschehen, wenn in dieser Situation die gesamte Wissenschaft den Schluss gezogen hätte, die Finger von Nachhaltigkeit und Nachhaltigkeitsforschung zu lassen? Das umfangreiche Wissen, das wir heute haben, um nachhaltige von weniger nachhaltigen Entwicklungen unterscheiden und begründet Maßnahmen zugunsten nachhaltiger Entwicklung entwickeln zu können (Grunwald/Kopfmüller 2012), wäre nicht erarbeitet worden. Diese Analogie mag Ansporn sein, es mit der Responsible Innovation ähnlich zu halten: In aller Anerkennung der Begriffsprobleme nicht puristisch abseits stehen, sondern sich aktiv darum bemühen, durch konzeptionelle Analyse, vor allem aber durch Praxis und das reflektierende Lernen aus dieser Praxis den Begriff der Responsible Innovation zu formen und zunehmend mit Inhalt zu füllen. 


\section{Literatur}

Aichholzer, G., Bora, A., Bröchler, S., Decker, M., Latzer, M. (Hg.), 2010, Technology Governance. Der Beitrag der Technikfolgenabschätzung. Berlin: Edition Sigma.

Bechmann, G., 1993, Ethische Grenzen der Technik oder technische Grenzen der Ethik? Geschichte und Gegenwart. Vierteljahreshefte für Zeitgeschichte, Gesellschaftsanalyse und politische Bildung 12, S. 213-225.

Beck, U., 1988, Gegengifte. Die organisierte Unverantwortlichkeit, Frankfurt: Suhrkamp.

Bijker, W.E., Hughes, T.P., Pinch, T.J. (Hg.), 1987, The Social Construction of Technological Systems. New Directions in the Sociology and History of Technological Systems. Cambridge (Mass.): Cambridge University Press.

Bijker, W.E., Law, J. (Hg.), 1994, Shaping Technology/Building Society. Cambridge (Mass.): Cambridge University Press.

Boxsel, J. A. M., 1991, Konstruktive Technikfolgenabschätzung in den Niederlanden. In: Kornwachs, K. (Hg.): Reichweite und Potential der Technikfolgenabschätzung. Stuttgart: Metzler, S. 137-154.

Dolata, U., Werle, R. (Hg.), 2007, Gesellschaft und die Macht der Technik. Sozioökonomischer und institutioneller Wandel durch Technisierung. Frankfurt/New York: Campus.

Durbin, P., Lenk, H. (Hg.), 1987, Technology and Responsibility. Dordrecht: Reidel Publishing

EC - European Commission, 2008, Commission Recommendation on a code of conduct for responsible nanosciences and nanotechnologies research. 2008/424 final, 7 February 2008. European Commission, Brussels.

Grunwald, A., 1999, Verantwortungsbegriff und Verantwortungsethik. In: Grunwald, A. (Hg.): Rationale Technikfolgenbeurteilung. Konzeption und methodische Grundlagen. Berlin et al.: Springer, S. 172-195.

Grunwald, A., 2010, Technikfolgenabschätzung. Eine Einführung. Berlin: Edition Sigma.

Grunwald, A., 2011, Synthetische Biologie. Verantwortungszuschreibung und Demokratie. Information Philosophie 5/2011, S. 19-19.

Grunwald, A., 2012, Technology Assessment for Responsible Innovation. In: J. van den Hoven (Hg.): Responsible Innovation. Proceedings of the Conference at The Hague, April 18/19 April, 2011 (in Vorbereitung).

Grunwald, A., Kopfmüller, J., 2012, Nachhaltigkeit. Frankfurt/New York, 2. Aufl.: Campus Verlag.

Habermas, J., 1968, Verwissenschaftlichte Politik und öffentliche Meinung. In: Habermas, J. (Hg.): Technik und Wissenschaft als Ideologie. Frankfurt: Suhrkamp, S. 120-145

Ilulissat Statement, 2008, Synthesizing the Future. A vision for the convergence of synthetic biology and nanotechnology. Views that emerged from the Kavli Futures Symposium 'The merging of bio and nano: towards cyborg cells', 11-15 June 2007, Ilulissat, Greenland.

Jonas, H., 1979, Das Prinzip Verantwortung. Versuch einer Ethik für die technologische Zivilisation. Frankfurt: Suhrkamp

Lenk, H., 1992, Zwischen Wissenschaft und Ethik. Frankfurt: Suhrkamp. 
National Research Council, 2006, A Matter of Size: Triennial Review of the National Nanotechnology Initiative.Washington, D.C.: National Academies Press.

Rapp, F. (Hg.), 1999, Normative Technikbewertung. Wertprobleme der Technik und die Erfahrungen mit der VDI-Richtlinie 3780. Düsseldorf: VDI-Verlag.

Rip, A:, T. Misa, J. Schot (Hg.), 1995, Managing Technology in Society. London/New York: Sage.

Ropohl, G., 1996, Ethik und Technikbewertung. Frankfurt am Main: Suhrkamp.

Schot, W., 1992, Constructive Technology Assessment and Technology Dynamics: The Case of Clean Technologies. In: Science, Technology and Human Values 17, S. 36-56.

Schot, J., Rip, A., 1997, The Past and Future of Constructive Technology Assessment. Technological Forecasting and Social Change 54, S. 251-268.

Schwarz, M., 1992, Technology and Society: Dilemmas of the Technological Culture. Technology and Democracy, Proceedings of the $3^{\text {rd }}$ European Congress on Technology Assessment. Copenhagen, S. 30-44.

Siune, K., Markus, E., Calloni, M., Felt, U., Gorski, A., Grunwald, A. Rip, A., de Semir, V., Wyatt, S., 2009, Challenging Futures of Science in Society. Report of the MASIS Expert Group. Brussels, European Commission.

Steinmüller, K.; Tacke, K. und Tschiedel, R., 1999, Innovationsorientierte Technikfolgenabschätzung. In: Bröchler et al. (Hg.): Handbuch Technikfolgenabschätzung, Berlin, Edition Sigma, S. 129-147.

van de Poel, I., 2009, Values in Engineering Design. In: A. Meijers (Hg.): Philosophy of Technology and Engineering Sciences. Volume 9. Amsterdam, p. 973-1006.

VDI - Verein Deutscher Ingenieure, 1991, Richtlinie 3780 Technikbewertung, Begriffe und Grundlagen. Düsseldorf: VDI-Verlag.

Yoshinaka, Y., C. Clausen und A. Hansen, 2003, The Social Shaping of Technology: A New Space for Politics? In A. Grunwald (Hg.), Technikgestaltung: zwischen Wunsch oder Wirklichkeit, Berlin: Springer, S. 117-131. 


\title{
Technikfolgenabschätzung als Ressource von Technology Governance
}

\author{
Georg Simonis \\ Institut für Politikwissenschaft, FernUniversität in Hagen
}

\section{Keywords}

Technikfolgenabschätzung, Technology Governance, Technologische Regime, Differenzierungstheorie, Koordination technologischer Entscheidungen.

\begin{abstract}
Technikfolgenabschätzung (TA) könnte für die an Technology Governance (TG) beteiligten Akteure eine bedeutende Wissensressource bilden. In diesem Beitrag wird untersucht, wie TG und TA aufeinander bezogen sind, um auf einer konzeptionellen Ebene zu ermitteln, wie über die Verbesserung von TA-Angeboten die Leistungsfähigkeit von TG erhöht werden könnte. Dabei soll erstens begründet werden, dass der Governanceansatz im Anwendungsbereich der Technikentwicklung und -gestaltung differenzierungstheoretisch ausgelegt sein sollte. Zweitens wird argumentiert, dass TA als ein normales Element von TG zu begreifen ist. TA und TG bilden keine getrennten Welten, sondern eine Einheit, in der TA ein mehr oder weniger ausdifferenziertes Arsenal von Entscheidungshilfen bereitstellt. Solange es kein rechtlich vorgeschriebenes Gebot zur Nutzung von TA-Analysen gibt, bleiben sie eine Wissensressource, von der Entscheidungsträger Gebrauch machen können oder eben auch nicht.
\end{abstract}

\section{Technology Assessment as a Resource of Technology Governance}

Keywords: Technology assessment, technology governance, technological regimes, differentiation theory, coordination of technological decisions.

\footnotetext{
Abstract: 'Technology Assessment (TA) could be an important resource of knowledge for the actors of Technology Governance (TG). This article analyses how TG and TA are intertwined. It aims at increasing the performance of TG by improving the offers of TA. It will be argued, firstly, that the governance approach should be based on differentiation theory in the practical field of developing and designing technology. Secondly, it is shown that TA can be understood as a normal element of TG. TA and TG are not worlds apart but form an entity in which TA provides a more or less differentiated arsenal of criteria helping in the decision-making process. As long as there is no legal obligation to use TA analyses, they remain a resource of knowledge which decision-makers might use or might not use.
} 


\section{Einleitung}

Für die an Technology Governance (TG) beteiligten Akteure bildet Technikfolgenabschätzung (TA) eine bedeutende Wissensressource oder könnte es, falls der TA-Instrumentenkasten den Akteuren zur Verfügung stünde. Nur in Ausnahmefällen ist dies aber der Fall. In dem Beitrag soll untersucht werden, wie TG und TA aufeinander bezogen sind, um auf einer konzeptionellen Ebene zu ermitteln, wie über die Verbesserung von TA-Angeboten die Leistungsfähigkeit von TG erhöht werden könnte. Diese Fragestellung erfolgt vor dem Hintergrund eines steuerungstheoretischen Grundverständnisses von Governance und speziell von TG.

Der Beitrag möchte zwei Thesen erläutern. Es wird im ersten Argumentationsschritt zu begründen versucht, dass der Governanceansatz auch im Anwendungsbereich der Technikentwicklung und -gestaltung differenzierungstheoretisch ausgelegt sein sollte. Die Aufspaltung der modernen Gesellschaft in funktionale Teilsysteme kann vom Governanceansatz nicht rückgängig gemacht werden. Daher ist es sinnvoll, zwischen politischer Technology Governance und TG in der Wissenschaft, der Wirtschaft und der Gesellschaft zu unterscheiden. Nachfolgend wird argumentiert, dass TA als ein normales Element, das allerdings auf sehr unterschiedlichen Niveaus entwickelt sein kann, von Technology Governance zu begreifen ist. Technology Assessment und Technology Governance bilden keine getrennten Welten, hier die Welt der Gestaltungsnormen, dort die Welt der realen Technikerzeugung, sondern eine Einheit, in der TA ein mehr oder weniger ausdifferenziertes Arsenal von Entscheidungshilfen darstellt. Die Governanceperspektive unterstützt diese integrative Deutung von TA. Aus governancetheoretischer Sicht lassen sich die normativen und realen Aspekte der Entwicklung sozio-technischer Systeme analytisch nicht trennen. Mit dieser governancetheoretischen Ausleuchtung von TA wird sie zu einer möglichen Ressource und als ein wissensbasiertes Instrument begriffen, das zur Governance sozio-technischer Systeme entscheidende Beiträge leisten kann.

Die Argumentation entfaltet sich in vier Schritten. Zunächst wird in der gebotenen Kürze der Stand der deutschen Diskussion zum Verhältnis TG und TA anhand der Arbeiten von Stephan Bröchler und Stefan Kuhlmann rekapituliert. Dann wird TG differenzierungstheoretisch konzipiert und definiert, um nachfolgend TA auf die jeweiligen Problemlösungskapazitäten von TG in unterschiedlichen gesellschaftlichen Systemen zu beziehen. Im dritten Abschnitt wird als Beispiel für die differenzierungstheoretische Deutung von TG auf das Konzept der politischen TG eingegangen. Der vierte Argumentationsschritt beschäftigt sich dann mit TA als einer Ressource, einem besonderen methodischen Instrument, zur Steigerung der Leistungsfähigkeit von TG. Ein knappes Fazit beschließt die Ausführungen.

\section{TG und TA in der Diskussion}

Stephan Bröchler $(2008,2010)$ begreift Technology Governance als „,das Zusammenwirken von Unternehmen, Wissenschaft, Staat und Gesellschaft für die Entwicklung und Nutzung technischer Innovationen“ (Bröchler 2010, 63) und unterscheidet zwischen einer normativen und einer analytischen Verwendung des Konzepts. Im normativen Kontext werde TG als Ansatz benutzt, eine „Erfolg verheißende Strategie“ effektiverer Techniksteuerung gegenüber der riskanten alltäglichen Nutzung von Technologien zu ermöglichen (S. 70). Dagegen betone die analytische Variante des Konzepts eine Forschungsperspektive. Mithilfe des Begriffs Technology Governance solle „die ver- 
änderte Realität der Rolle des Staates in der Steuerung und Koordination der Entwicklung und Nutzung technischer Innovationen“ (S. 70) besser erklärt werden. Technikfolgenabschätzung habe dagegen einen anderen Fokus. TA sei ,ein ausdifferenziertes reflexives System, das Wissen für die Bearbeitung öffentlicher Technikprobleme ... erzeugt, bewertet und das Wissen für die Kontextualisierung von Technik bereitstellt“ (S. 70). Technikfolgenabschätzung und Technology Governance seien als unterschiedliche Problembearbeitungsmechanismen zu begreifen, die allerdings ,,sinnvoll aufeinander bezogen werden können“ (S. 71-72). Die beiden Problembearbeitungsmodi stehen für Bröchler in einem spannungsreichen Über- und Unterordnungsverhältnis. TG beschreibt generell die Regelungsstruktur des Prozesses technischer Innovationen. TA dagegen stellt ein reflexives System zur besseren Bearbeitung öffentlicher Technikprobleme dar. Somit könne TA, ausgebaut als ein spezielles Governancesystem, einen reflexiven Beitrag zu der Governance von Technologien leisten. Damit dies gelinge, bedürfe es ,,in Zukunft erheblicher weiterer interdisziplinärer Forschungsanstrengungen“" (S. 72).

Auch Stefan Kuhlmann (2010) sieht Technology Governance und TA als zwei getrennte Handlungsfelder. Er unterscheidet streng zwischen der realen Governance sozio-technischer Systeme und Instrumenten, die zu einer Verbesserung der Techniksteuerung beitragen könnten. Um die Differenz von Realität und korrigierender politischer Intervention zu betonen, spricht er von der „De-facto-Governance soziotechnischer Regimes“, die sich als „Gewebe kognitiver, normativer und regulatorischer Regeln begreifen“ (S. 48) ließen. Im Verhältnis zur De-facto-Governance bilde TA einen modulierenden Faktor, ein „Mittel reflexiver Governance“. Nach dieser Klärung der Verhältnisse ergibt sich für Stefan Kuhlmann die zwingende Folgerung: „Je besser wir die De-factoGovernance eines gegebenen Regimes ... verstanden haben, desto eher kann TA realistisch ... als, 'einwirkende' Intervention erfolgen ..." (S. 48).

Beide Konzeptionen des Verhältnisses von TG zu TA, die den aktuellen Diskussionsstand zu repräsentieren scheinen, betonen deren Unterschiede und trennen zwischen der empirischen Analyse der Real-Welt (De-facto-Governance) sowie den normativen Ansprüchen, die Nebenwirkungen von Technologien zu vermeiden. Mir scheinen beide Annahmen zu rigide zu sein und den gegenwärtigen Verhältnissen von Technology Governance nicht gerecht zu werden:

1. TG rekonstruiert die Bewältigung von Problemen, die durch die Entwicklung, Anwendung und die Entsorgung technischer Systeme erzeugt werden. An diesen Problembewältigungsprozessen sind zahlreiche Akteure mit unterschiedlichsten Handlungskapazitäten und Instrumenten beteiligt. Eine bestimmte Klasse von Instrumenten ist insbesondere damit beschäftigt, die Entstehung unerwünschter Probleme zu verhindern, Technologien hinsichtlich möglicher Risiken zu bewerten und sie in einer Weise zu gestalten, dass Risiken minimiert werden. TA ist ein Instrument dieser Klasse von Aktivitäten, ist also ein Teilregime der Entwicklung und Anwendung von Technik, das je nach Gesellschaft und Technologie sehr unterschiedlich entwickelt sein kann. Betrachtet aus der Perspektive der Technology Governance muss TA als ein Verfahren, in dem Entscheidungsträger und gesellschaftliche Akteure beraten werden, begriffen werden. Um eine höhere Nachhaltigkeit der Techniknutzung zu erreichen, werden Prozesse der Technikbewertung und Technikgestaltung unterstützt. TA ergänzt andere Bewertungsverfahren, wobei die Übergänge zwischen den Bewertungsverfahren der Ingenieure in den Entwicklungsabteilungen, der Marketingexperten, der Risikospezialisten in den Versicherungen und von interdisziplinären TA-Teams, die das öffentliche Interesse einbringen, fließend sind. Technikbewertung findet allenthalben statt, „nur“ die Kriterien, Methoden und Verfahren unterscheiden sich.

2. Im Rahmen des Prozesses der Technology Governance, verstanden als „De-facto-Governance“ im Sinne Kuhlmanns, werden kontinuierlich Entscheidungen gefällt, die den Normen der Regelungsstrukturen folgen. Der Technikforscher, der diese Entscheidungen rekonstruiert, bleibt nicht unbeteiligt. Seine Forschungsergebnisse fließen, gewollt oder ungewollt, in den gesellschaftlichen, wissenschaftlichen und politischen Prozess der Technikbewertung ein. Weder lässt sich 
die strikte Unterscheidung zwischen der analytisch verfahrenden und der normativ ausgerichteten Governanceforschung, noch die deutliche (logische) Trennung zwischen analytischer Governanceforschung und normativ angelegter TA-Forschung bei einer empirischen Betrachtung der Verhältnisse durchhalten. Die analytische Governanceforschung enthält nicht hintergehbare normative Elemente. Allein die von ihr verfolgte Problemlösungsperspektive beinhaltet eine starke normative Festlegung. Die Differenzen sind also auch in der normativen Dimension nur gradueller Natur.

Das im Weiteren entwickelte Governancekonzept unterscheidet sich von den beiden, hier nur kursorisch skizzierten, Ansätzen. Es verfolgt eine ganzheitliche Betrachtungsperspektive, in der die analytische Dimension nicht künstlich von der Welt der Normen getrennt wird und in der die Instrumente und Verfahren der Technikfolgenabschätzung ein normales, immer präsentes, aber mal weniger, mal stärker ausdifferenziertes Moment der Bewältigung sozio-technischer Probleme darstellen.

\section{TG differenzierungstheoretisch betrachtet}

Die Governanceanalyse bezieht sich auf Institutionen. Sie interessiert sich für Institutionen, die mehr oder weniger autonome, aber interdependente Akteure zur Bewältigung gemeinsamer Probleme zu koordinieren vermögen (siehe Benz et al. 2007). Im Zentrum von Technology Governance stehen daher Institutionen und Akteure, die gemeinsam Probleme bearbeiten, die bei der Entwicklung und Reproduktion soziotechnischer Systeme, die sich auch als Regime begreifen lassen, auftreten. In den Fokus einer governanceanalytischen Untersuchung gelangen:

- die Probleme, die zu bewältigen sind und die von den Akteuren zuvor als Probleme wahrgenommen und anerkannt werden müssen,

- die Akteure mit ihren Handlungskapazitäten, Zielen, Normen und Strategien,

- die Koordinationsformen und Koordinationsmechanismen,

- das Problemlösungshandeln, der Impact (Effektivität und Effizienz) und die Art der Reproduktion des Governancesystems mit oder ohne Lerneffekte.

Diese abstrakten Definitionen von Governance wie von Technology Governance geben noch keine Hinweise auf die Art der Institutionen und ihre gesellschaftliche Einbettung. Mit einer systemtheoretischen oder differenzierungstheoretischen Betrachtungsweise lassen sich Institutionen funktional differenzierten Systemen zuordnen. Dabei ist zu beachten, dass Institutionen in der Form von Organisationen mit der Eigenschaft korporativer Akteure mehreren funktional ausdifferenzierten Systemen angehören können. Bspw. unterliegen Unternehmen primär den Handlungszwängen (Kommunikationscodes) des Wirtschaftssystems. Sie sind aber auch politische Akteure in ihrer Eigenschaft als „Rule takers“ gesetzlicher Vorschriften und „Rule makers“, die sich an der Entstehung gesetzlicher Normen beteiligen ${ }^{1}$.

Die, ein von seiner Umwelt differenziertes System definierende und seine Leistungen bestimmende, Funktionslogik (Kommunikationslogik im Sinne Luhmanns) findet sich in den Institutionen des Systems als normative Anforderung gegenüber den Akteuren, die sich den Spielregeln der institutionalisierten Regelungsstruktur und somit auch der Systemlogik fügen. Am Beispiel des politi-

1 Zur Begrifflichkeit vergleiche Streeck (2009, 239). 
schen Systems lässt sich dieser Zusammenhang verdeutlichen: Die Institutionen des politischen Systems werden mit Luhmann (vgl. Luhmann 2008) vom Kampf um die politische Macht, verstanden als Kampf um die Positionen, die mit der Befugnis verbunden sind, an kollektiv bindenden Entscheidungen mitzuwirken, geprägt. Akteure, die im Rahmen politischer Institutionen handeln, also von Institutionen, die ihrerseits den Kampf um die Besetzung der Positionen, die an kollektiv bindenden Entscheidungen beteiligt sind, regulieren, unterliegen den Handlungsanforderungen des politischen Systems. Auch die Handlungsprogramme ${ }^{2}$, mit denen sich die Politik in Bezug auf die Gesellschaft in vielen Handlungsfeldern (Politikbereichen) organisiert, unterliegen der Machtlogik des politischen Systems. Ihre Regelungen müssen, damit sie Gesetzeskraft erlangen, die Abstimmungsprozeduren des legislativen Systems erfolgreich durchlaufen. Sie müssen auch über ein Mindestniveau an Output-Legitimität verfügen, damit sie von den „Rule takers“ akzeptiert werden, wobei unter demokratischen Verhältnissen spätestens bei der nächsten Wahl über das erforderliche Zustimmungsniveau befunden wird. Politische Handlungsprogramme etablieren und reflektieren politische Regelungsstrukturen (Institutionen), die ihrerseits das Handeln der „Rule takers“, soweit diese sich an die Regeln halten, regulieren und koordinieren.

Aus einer differenzierungstheoretischen Perspektive betrachtet, sind die Institutionen, die sich mit TG befassen, je nach dem, in welches Funktionssystem - Politik, Wirtschaft, Wissenschaft, Gesellschaft - sie integriert sind, unterschiedlichen Systemanforderungen ausgesetzt. Diese je spezifischen Anforderungen sollten, oder genauer: müssen, bei der Analyse von TG beachtet werden. Abbildung 1 verdeutlicht diese Argumentation:

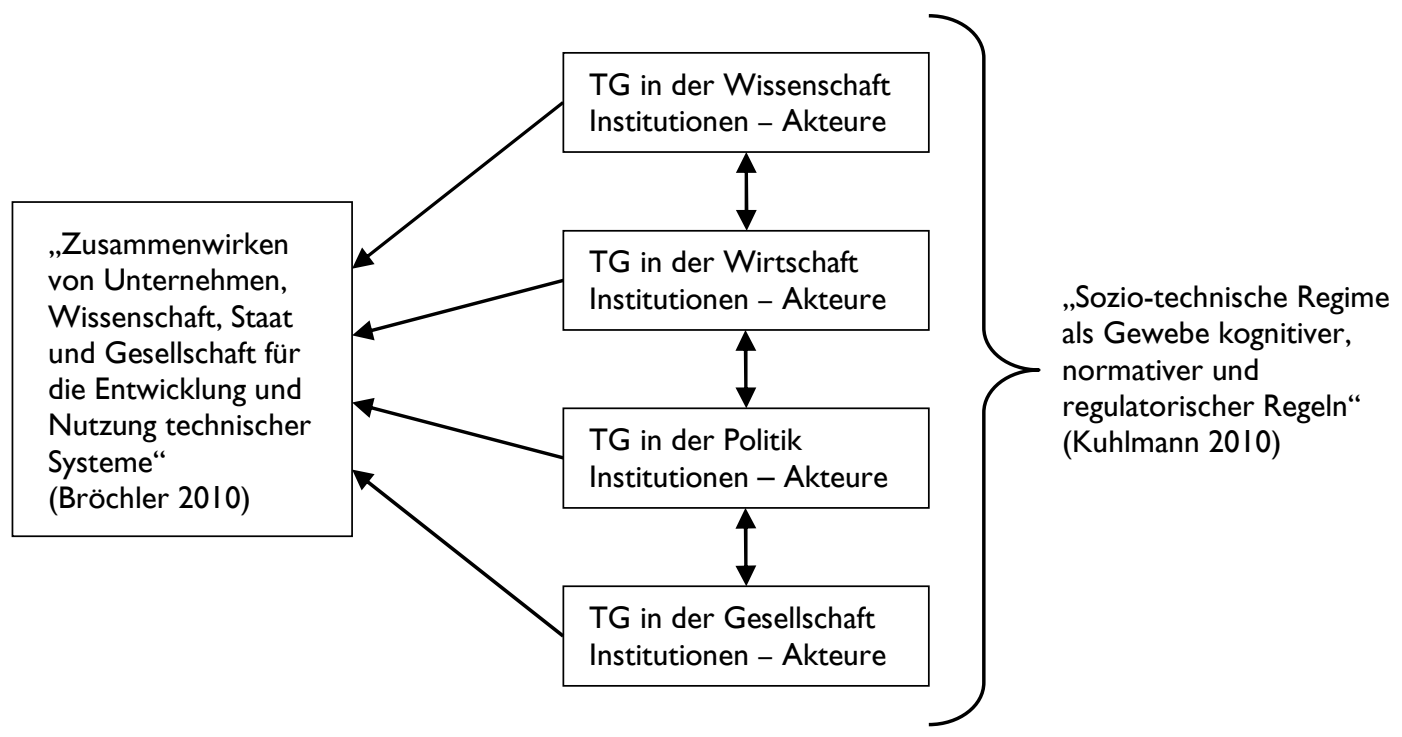

Abbildung 1: Differenzierungstheoretische Analyse von TG (Quelle: GS)

2 Luhmann $(2008,60)$ selbst führt diese Unterscheidung zwischen Codierung und Programmierung, zwischen Werten und Programmen, ein. Luhmanns Definition von Programmen $(, \ldots$ Programme sind ... vorgegebene Bedingungen für die Richtigkeit der Selektion von Operationen“) entspricht der hier verwendeten Definition von Institutionen. 
TG erfolgt also primär durch Akteure, die den Restriktionen und Anforderungen von Governanceinstitutionen gerecht werden müssen und sekundär durch Institutionen, die ihrerseits in gesellschaftliche Funktionssysteme integriert sind. Dieser Strukturaufbau ermöglicht, dass die von TG vorgeschlagenen Problemlösungen funktionalen Anforderungen entsprechen und vom betroffenen System dekodiert und verarbeitet werden können. Problemlösungen müssen systembezogen realistisch sein. Lösungsansätze, die in der Wissenschaft beispielsweise akzeptabel sind, da sie der herrschenden Lehrmeinung nicht widersprechen, also für - zumindest vorläufig - wahr gelten, können in der Politik scheitern, sofern sie im Kampf um die Macht die „falsche“ Seite stärken. Im Repräsentantenhaus der USA haben die klimaskeptischen Republikaner seit den Wahlen vom Herbst 2010 eine Mehrheit. Mit dem Argument, dass es den anthropogen verursachten Klimawandel nicht gebe, verhindern sie die Ausarbeitung eines Klimaprogramms und eine entsprechende Gesetzgebung (vgl. Simonis 2011). Dass ihre Position von der Zunft der Klimaforscher nicht geteilt wird, schadet ihnen nicht. Im Gegenteil. Die Skepsis gegenüber dem Stand der Klimaforschung fördert den Stimmenfang und sichert politische Macht.

Auf der Grundlage des differenzierungstheoretisch präzisierten Konzepts von Technology Governance können nun die Problemstellen der TG-Konzepte von Bröchler und Kuhlmann deutlicher erkannt werden. Im Ansatz von Stephan Bröchler wirken die Akteure - siehe die Definition oben (Schaubild 1) - unmittelbar zusammen. Die Institutionen und die Systeme, in denen sie operieren, werden ausgeblendet. Diese Dekontextualisierung der Akteure hat einen beachtlichen Verlust an gesellschaftlicher Komplexität zur Folge. Die Akteure von TG haben die in den Strukturen eingeschriebenen, allerdings auch veränderbaren, Restriktionen und Anreize, zu beachten, wenn sie denn erfolgreich sein wollen. TG innerhalb des politischen Systems erfolgt nach anderen Spielregeln als TG in der Wissenschaft, der Wirtschaft oder der Gesellschaft. Die Hoffnung, dass der TG-Ansatz gesellschaftliche Differenzierungen aufheben könnte, trügt. Der Einwand, dass in heutigen Zeiten Akteure unterschiedlicher Systeme immer mehr zusammenarbeiten, ist eine wichtige empirische Beobachtung, die aber nicht die Missverständnisse und Enttäuschungen erklären kann, die sich häufig bei projektorientierter, netzwerkförmiger und direkter Kommunikation einstellen, weil die Partner unterschiedlichen Institutionen (Programmen) und Systemen angehören. Auch die wissenschaftliche Politikberatung ${ }^{3}$ vermag die Differenz zwischen Politik und Wissenschaft nicht zu überwinden, obwohl es, wenn die Wissenschaft eine politische Rolle übernimmt, so scheinen könnte. Ein interessantes Beispiel dafür, wie sich Systemgrenzen überschneiden, liefert die beratende Klimaforschung (Beck 2009, Beck 2011, Bolin 2007). Der Weltklimarat (IPCC), der den wissenschaftlichen Konsens der Klimaforschung für die Politik zusammenfasst, der Handlungsanforderungen formuliert und der somit Teil des politischen Systems ist, bewegt sich in zwei Systemen. Aus der Sicht der Politik ist der IPCC zu einem wichtigen politischen Akteur geworden, obwohl viele seiner Mitglieder „nur“ Klimaforscher sein möchten, die sich der wissenschaftlichen Wahrheit verpflichtet sehen. Damit die Aussagen des IPCC in Politik und Gesellschaft auf Resonanz stoßen, dürfen sie nicht politisch kodiert sein oder gar einen politischen Bias besitzen. Sie müssen im wissenschaftlichen Sinne wahr sein, obwohl die Politik an der Zusammenfassung für politische Entscheidungsträger mitwirkt.

Im Zentrum des Governanceansatzes von Stefan Kuhlmann stehen nicht die Akteure, sondern sozio-technische Regime. Er folgt der Definition von Ende/Kemp (1999), die von Rip/Kemp (1998) und Geels/Schot (2007) zu einer Heuristik der soziotechnischen Transition, so Kuhlmann (2010, 46), erweitert wurde. In diesem Verständnis werden technische Systeme als soziotechnische Institutionen begriffen, also als Regime, die eine Geschichte haben und Ergebnis strategischer Interventionen und von Institutionalisierungsprozessen sind. Auf die Entstehung und Entwicklung soziotechnischer

3 Zur Entwicklung der wissenschaftlichen Politikberatung vgl.Weingart/Lentsch (2008), Bröchler/Schützeichel (2008). 
Regime hat die Politik nur beschränkten Einfluss. Mit dem Konzept der De-facto-Governance bezeichnet Kuhlmann die Interaktionen zwischen einem soziotechnischen Regime und den politischen Governancestrukturen. Er spricht von der Modulierung des Regimes durch die Politik. Der Versuch die beiden institutionalistischen Analyseansätze: Governance und soziotechnische Regime aufeinander zu beziehen, ist plausibel. Er leidet aber unter einer institutionalistischen Blindstelle, der Machtvergessenheit vieler institutionalistischer Analysen, einschließlich der Governanceanalyse. Dieser Schwachpunkt lässt sich mit Rückgriff auf differenzierungstheoretische Konzepte vermeiden. Wir haben es nicht nur mit etablierten politischen Handlungsprogrammen als Institutionen zu tun, sondern auch mit ausdifferenzierten Systemen, die gleichfalls als Institutionen gelten können und die den Programmen eine jeweils spezifische Richtung verleihen. Akteure, die sich in der Sphäre der Politik nicht an den Code der Politik halten, werden dort bestenfalls als Hofnarren geduldet sein.

\section{Politische Technology Governance}

Soll das Analyseniveau von TG dem die postfordistische Wissensgesellschaft auszeichnenden Komplexitätsgrad entsprechen, führt kein Weg daran vorbei, zwischen unterschiedlichen, systembedingten Varianten von TG zu unterscheiden. Politische Technology Governance befasst sich mit der politischen Governance von Technologien, also mit der Bewältigung von politischen Governanceproblemen, unter dem doppelten Blickwinkel des internen politischen Konsensbildungsprozesses und der Output-Legitimität der zu beschließenden Handlungsprogramme (gesetzlichen Regelungen). Welches sind aber die politischen Probleme, die zum Gegenstand politischer TG werden können? Eine analytisch-abstrakte Antwort würde allein auf den Prozess der Politisierung technikbedingter Probleme und auf deren Stellenwert für die Reproduktion von Machtpositionen abstellen. Eine etwas weniger formale Antwort kann zwei zusätzliche Gesichtspunkte hervorheben: Governanceprobleme entstehen für das politische System aus Differenzen zwischen Normen etablierter Programme und deren (drohender) Verletzung, sei es (1) durch externe politische Ereignisse, sei es (2) durch interne Entwicklungen, die die Konsistenz zwischen institutionalisierten Programmen gefährden. Da sich die politischen Systeme, zumal unter demokratischen Verhältnissen, abgesehen von der prozeduralen (legalen) Legitimität, sowohl der Input- wie auch der Output-Legitimität ihrer Entscheidungen versichern müssen, werden Problemlösungen im Lichte ihres Beitrags zur Sicherung des Gemeinwohls begründet. Dem Publikum, den „Rule takers“, ist es ziemlich egal, ob die erlassenen Regelungen des Gesetzgebers, die Machtpositionen der Mehrheitsfraktion sichern oder gefährden. Die Kunst des Regierens besteht in der optimalen Verbindung von Macht- und Sachlogik.

In Anlehnung an Streeck/Thelen (2005) und Streeck (2009, 239) lassen sich die Strukturbedingungen politischer TG in einem einfachen Schema (Abbildung 2) zusammenfassen. Auch hier wird auf das Regimekonzept zurückgegriffen. Jedoch wird von Streeck und Thelen ein gänzlich anderer Sachverhalt mit dem Konzept analysiert. Wiederum geht es allerdings um die Untersuchung von Institutionen. In diesem Falle aber um politische Institutionen, deren Entstehung sich gesetzgebender Akte, die auch Verwaltungshandeln einschließen, verdankt und die mit politischer Legitimität ausgestattet sind. Ein politisches Regime besteht nach dieser Konzeption aber nicht alleine aus der Regelungsstruktur, sondern auch aus den Akteuren, die Regeln setzen, den ,Rule makers“, und den Akteuren, die Regeln zu befolgen haben, den ,Rule takers“, insgesamt eingebettet in einen nationalgesellschaftlichen Kontext. 


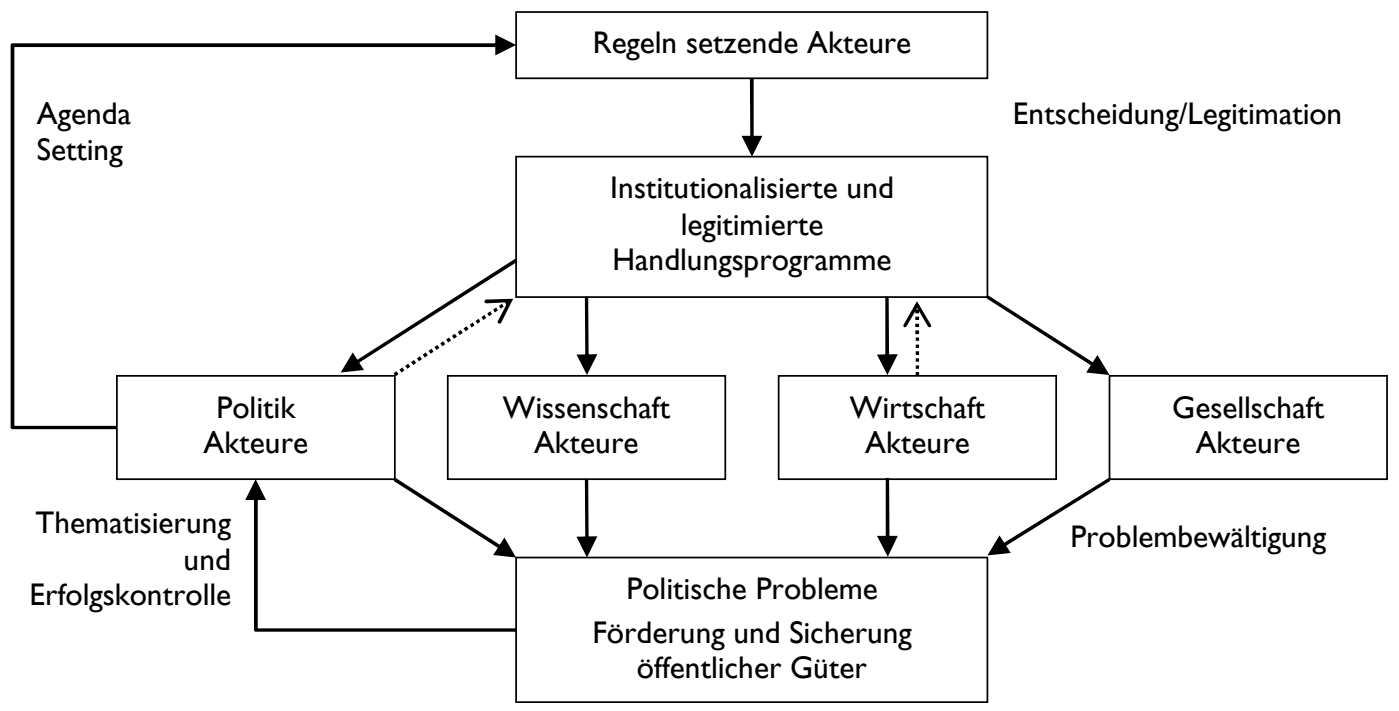

Abbildung 2: Strukturschema politischer Technology Governance im Rahmen eines sozio-technischen Regimes (Quelle: GS)

In einer governanceanalytischen Erweiterung dieses politischen Regimeansatzes, dem es darum geht, inkrementellen institutionellen Wandel mit transformatorischen Konsequenzen ${ }^{4}$ zu erklären, wird zusätzlich auf die politischen Probleme abgestellt, die von dem Regime bearbeitet und irgendwie bewältigt werden. Im Kontext von TG werden technikinduzierte Probleme mit Hilfe staatlich legitimierter Institutionen, die das Handeln der von ihnen betroffenen Akteure aus Wirtschaft, Gesellschaft, Wissenschaft, Politik und Verwaltung koordinieren und steuern, geregelt ${ }^{5}$. Als Beispiel für eine solche Governancestruktur sei auf das deutsche Atomgesetz von 1960 verwiesen. Das Gesetz führte zur Etablierung eines politischen Regimes zur Regelung aller Probleme der zivilen Nutzung der Kernkraft, von denen der damalige Gesetzgeber meinte, sie sollten, weil es ein politisch artikuliertes - öffentliches - Interesse gebe, staatlich geordnet werden. Aus governancetheoretischer Sicht wurde zur Bewältigung eines bestimmten Sets an Problemen der Nutzung der Kernkraft eine komplexe institutionelle Struktur, die sich sinnvoll als Regime charakterisieren lässt, etabliert, die seitdem die betroffenen Akteure zur Koordination ihres Handelns verpflichtet und die daraufhin zu befragen ist, in welcher Weise die Akteure ihren Verpflichtungen nachkommen, in welcher Richtung sich das Regime weiterentwickelt und ob die Governance der Technologie die in sie gesetzten Erwartungen erfüllt.

Politische TG lässt sich durch die folgenden Merkmale charakterisieren und ist damit leicht von der Governance soziotechnischer Regime unterscheidbar:

- Politische TG erfolgt, wie in der politischen Governanceanalyse generell, problemorientiert. Bearbeitet werden im Zusammenhang mit der gesellschaftlichen Kontextualisierung von Technologien stehende politische Probleme, soweit es dafür einen politisch artikulierten, zwingenden Bedarf gibt.

- Regelungsstrukturen (Programme, Institutionen) bilden die statische Voraussetzung für das Handeln der Akteure. Die Frage, ob die Akteure - koordiniert und gesteuert durch die Regelungs-

4 Siehe auch Dolata (2011), der sich gleichfalls auf den Regimeansatz von Thelen/Streeck (2005) zur Erklärung technologischer Transformationen bezieht.

5 Dabei sind die Governanceformen immer weniger hierarchisch angelegt, sondern eher netz- und marktförmig. 
struktur - die gemeinsam zu lösenden Probleme, tatsächlich und in welchem Ausmaß bewältigen, ist der zentrale Fokus der Governanceanalyse.

- Obgleich institutionalistische Analysen leicht einen ahistorischen Bias haben, kann Governanceanalyse mit Hilfe des Regimeansatzes temporalisiert werden und historische Tiefe erlangen (Differenzierung zwischen den „Rule makers“ und den „Rule takers“, Einbeziehung von Rückkopplungen ${ }^{6}$, Berücksichtigung von Wahrnehmungsdifferenzen, Handlungsressourcen, Diskursen und strategischem Handeln).

- Gegenstand von politischer TG sind Technologien, die sich aus sozialwissenschaftlicher Sicht als soziotechnische Regime beschreiben lassen. Diese Regime werden durch politische TG kontextualisiert, damit die technischen Produkte in den gesellschaftlichen Verkehr gebracht werden dürfen, die Gesellschaft, soweit dies politisch geboten erscheint, vor negativen Folgen geschützt wird und die Besitzer der Technologie in die Lage versetzt werden, ihr Recht auf Nutzung durchzusetzen.

- Politische TG ist zunehmend wegen wachsender Komplexität der wissenschaftlich-technischen Gesellschaft auf wissenschaftliche Beratung angewiesen. Die politischen Akteure, die ihre Machtund Entscheidungspositionen bewahren und jene, die sie erobern wollen, müssen regulative Entscheidungen über mehr oder weniger politisierte Tatbestände treffen, deren Folgen sie jedoch nicht übersehen können. Zur Konzipierung, inhaltlichen Begründung und formalen Legitimierung müssen sie wissenschaftlich-technischen Sachverstand mobilisieren. Dieser kann intern oder extern des politisch-administrativen Systems gewonnen werden. Wissenschaftlich-technische Experten in politischen Rollen (Politikberatung) sind daher ein unverzichtbares Moment politischer TG.

\section{TA als Ressource für Technology Governance}

Sofern die Beobachtung richtig ist, dass sich in globalisierten, post-fordistischen Wissensgesellschaften die politisch-administrativen Systeme zunehmend mit technologischen Problemen befassen, da der ungestörte Normalbetrieb Entscheidungen verlangt, die sich mit jeweils ungewissen Folgen für Politik und Gesellschaft auf die Entwicklung, Anwendung und Entsorgung von Technologien beziehen, gewinnt zur Reduktion von Unsicherheit wissenschaftliche Technikberatung der Politik quantitativ und qualitativ an Bedeutung. Dabei lassen sich grob vier Typen von Entscheidungssituationen unterscheiden: (1) Die Nutzung von soziotechnischen Systemen im engeren und weiteren Staatsbetrieb; (2) die Förderung der Entwicklung neuer Technologien; (3) die Einführung von soziotechnischen Innovationen; (4) die Neubewertung bekannter Technologien wegen neuer Erkenntnisse über deren Nebenwirkungen. Bei jeder dieser Entscheidungssituationen kann TA-Expertise gefragt sein. Die Hypothese hat einige Plausibilität, dass sich mit zunehmender Ungewissheit über die Folgen der Nutzung neuer Technologien die Wahrscheinlichkeit einer steigenden Nachfrage nach reflexiven Beratungsleistungen erhöht 7 . Eine Implikation dieser These wäre dann, dass gerade in technologisch führenden Staaten TA-Aktivitäten im Rahmen der politischen Governance von Technologien verbreitet sind.

6 Im Schaubild 2 sind einige dieser Rückkopplungen angedeutet, vor allem die Erfolgskontrolle (Monitoring) sowie die Thematisierung von Defiziten und das „Agenda Setting“ durch Medien, Verbände, einschlägige Akteure und Parteien.

7 Vgl. die Beiträge in Voss et al. (2006). 
Ein wichtiger modifizierender Faktor dieser These besteht in der Ausprägung von TA-Kapazitäten und TA-Aktivitäten im jeweiligen gesellschaftlichen Umfeld der politisch-administrativen Systeme (s. Abbildung 3). Technology Governance findet in kapitalistischen Wettbewerbsgesellschaften vor allem in der Wirtschaft statt. Da die Entwicklung vieler neuer Technologien und Innovationen wissensbasiert erfolgt, hat TG im wissenschaftlich-technischen System, das seinerseits mit dem Innovationssystem der Wirtschaft eng verbunden ist, gleichfalls einen wichtigen Stellenwert. Schließlich dürfen die Governanceleistungen der gesellschaftlichen Kräfte, insbesondere der Zivilgesellschaft, nicht unbeachtet bleiben. Ohne die Interventionen der Neuen Sozialen Bewegungen in die soziotechnischen Paradigmen, in die Wirtschaft und die Politik lassen sich die Verlaufsformen politischer TG in unterschiedlichen Ländern nicht erklären. Wir haben also folgende Situation: TG findet in mehreren gesellschaftlichen Teilsystemen statt, die nicht, wie Luhmann argumentiert, als kommunikativ abgeschlossen und autopoietisch selbstbezogen konzipiert werden sollten, sondern die sich wechselseitig beeinflussen und in spezifischer Weise interdependent sind ${ }^{8}$. In jedem der gesellschaftlichen Teilsysteme werden Governanceentscheidungen getroffen, die Governanceprobleme lösen sollen, die in anderen Teilsystemen aber auch Governanceprobleme erzeugen können. Die Ambivalenz soziotechnischer Systeme bildet sich in dieser offenen Situation ab. Alle systemisch geprägten und institutionell koordinierten Entscheidungen haben einen Impact auf die jeweilig bearbeitete Governancethematik, deren Wandel von den Akteuren der Teilsysteme jeweils aus ihrer systemischen Perspektive registriert wird. Daher muss davon ausgegangen werden, dass es nur in Ausnahmefällen zwischen und auch innerhalb der Teilsysteme zu einer konsensualen Deutung von Governanceproblemen kommt. Dissonanz ist der Normalfall.

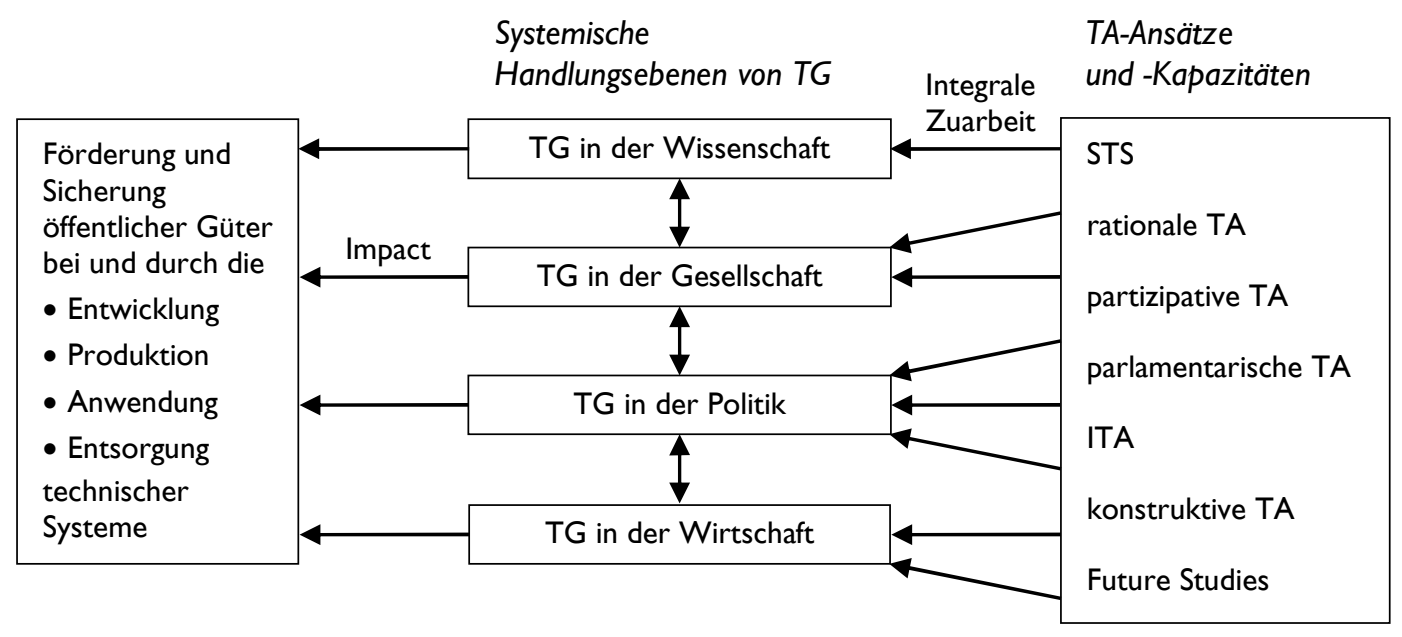

Abbildung 3: TA als Ressource von TG (Quelle: GS)

Die Dissonanz der Problemdeutung wirkt auf die Akteure in den Teilsystemen zurück und erzeugt zusätzlichen Klärungsbedarf, u. a. im politischen System, das institutionell unter dem Zwang steht, mehrheitsfähige Entscheidungen mit Output-Legitimität zu treffen. Erörtert unter einer funktionalistischen Perspektive gelangen bei dieser Abwägungs- und Entscheidungskonstellation die Leis-

8 Es bestehen zwei Typen von Interdependenzen: (1) Hinsichtlich der Akteure. Die Akteure sehen sich differenziellen Anforderungen unterschiedlicher Teilsysteme ausgesetzt und müssen versuchen, konsistente Handlungsstrategien zu finden. (2) Hinsichtlich der wechselseitigen Leistungen von Teilsystemen. Diese können Bestandsvoraussetzungen (z. B. Sicherheit, Recht) darstellen und/oder wichtige Inputs für andere Teilsysteme (Geld, Wissen, Akzeptanz) liefern. 
tungen der TA-Akteure in den Blickpunkt. Die unterschiedlichen Formen von TA scheinen geeignet, zum Abbau von Dissonanzen - der widersprüchlichen Deutungen von Governanceproblemen im Zusammenhang mit der Entwicklung und Nutzung von Technologien - passende Beiträge zu leisten.

Das Instrument der Technikfolgenabschätzung besteht grundsätzlich aus zwei Elementen (Simonis 2001): einer materialen Komponente, mit der Sachverhalte geprüft werden, und einer prozeduralen Komponente, mit der die abgeklärten Sachverhalte in Prozesse der TG eingespeist und an Akteure der TG vermittelt werden. TA richtet sich an Adressaten, die in irgend einer Weise an Governanceprozessen beteiligt und die sich nicht ganz sicher sind, wie soziotechnische Systeme genutzt und/ oder angelegt (gestaltet) werden sollten, damit öffentliche Güter nicht beschädigt, sondern gefördert und die Akzeptanz der Bürger gewonnen wird. Sofern sich „Science and Technology Studies“ (STS) und „Future Studies“ vorrangig an die einschlägige Wissenschafts-Community oder an eine anonyme Leserschaft ohne klaren Akteursbezug richten, bilden sie Grenzfälle von TA. Im Falle der partizipativen TA konkretisiert sich das Produkt, das an Entscheidungsträger zu vermitteln ist, in Ergebnisberichten über mögliche Konsensbildungsprozesse in unterschiedlich organisierten Beteiligungsgruppen. Falls diese direkt an Entscheidungsprozessen angekoppelt sind, kann partizipativ TA aber auch, entsprechend der parlamentarischen und der konstruktiven TA, zu einem prozeduralen Bestandteil von TG werden.

Die verschiedenen Formen und Konzepte von TA beziehen sich auf je spezifische gesellschaftliche Teilsysteme 9 und sind mal mehr, mal weniger in TG-Prozesse integriert. Dabei finden wir zwischen den Ländern und den sehr unterschiedlich strukturierten Politikfeldern, Wirtschaftsbranchen, gesellschaftlichen Nutzungskontexten und wissenschaftlichen Fachdebatten enorme Differenzen. Diese stehen hier nicht zur Diskussion. Es geht vielmehr um ein analytisches Argument: TA mit ihren differenten Ausprägungen bezüglich der gesellschaftlichen Teilsysteme steht nicht außerhalb der Governanceprozesse von Technologien. Die TA-Community ist weder ein unbeteiligter Beobachter, der über die Chancen und Risiken von Technologien forscht, ohne auf den Lauf der Dinge einwirken zu wollen, noch ein wissender Steuermann, der auf rauer See ein falsch orientiertes Schiff wieder auf Kurs bringt (Fach und Simonis 2000). Handlungsmächtige Adressaten werden von der TAForschung imaginiert und strategisch eingeplant oder sind die Auftraggeber, die Beratungsbedarf haben. Auf diese Konstellation hin ist TA zugeschnitten. Ob sie in konkreten Beratungssituationen den in sie gesetzten Erwartungen entsprechen kann, steht zwangsläufig auf einem anderen Blatt, zumal die Erwartungen der Adressaten in der Regel widersprüchlich sind.

In welchem Umfang in der Praxis Forschungs- und Beratungsleistungen der systematischen Folgenabschätzung von Technologien unter Gemeinwohl orientierten Kriterien, wie Nachhaltigkeit, Vorsorge und Demokratieverträglichkeit, in TG-Prozesse einfließen, kann nur mit vergleichender sozialwissenschaftlicher Forschung ermittelt werden. Das wäre für Technikforscher eine lohnende Aufgabe, die allerdings nur Früchte trägt, wenn zuvor geklärt ist, welche Forschungs- und Beratungsleistungen wie klassifiziert werden. Dass Abgrenzungen nicht ganz leicht, aber doch zu treffen sind, zeigen die zusammenfassenden Überlegungen von Grunwald (2010, 114-118). Seine breite Definition hilft, TA als einen wissenschaftlich basierten Beratungsprozess über die Folgen der Nutzung soziotechnischer Systeme zu begreifen, der heute in vielen Ländern eine breite Ausprägung erlangt hat und der somit nicht ,als einwirkende Intervention“ (Kuhlmann 2010) eines klugen Steuermanns, sondern als normale und widersprüchliche Praxis von TG in unterschiedlichen gesellschaftlichen Teilsystemen zu begreifen ist. Zur Aufklärung über die Defizite der Praxis von TG vermag TA-Forschung dann auch einen wichtigen Beitrag zu leisten.

9 Diese Ausführungen schließen an Lingner (2010) an. 


\section{Fazit}

Bleibt zu erörtern, ob TA eine spezielle Form von TG ist. Nach den voranstehenden Ausführungen bietet sich diese Schlussfolgerung nicht an. TG bezieht sich auf Regime und Institutionen, die im Bereich der Politik - andere Funktionssysteme wurden nicht genauer betrachtet - einen formalen und damit legitimierten Charakter besitzen. Informale Institutionen sollten hier wegen zu geringer politischer Verbindlichkeit ausgeschlossen sein. TA ist ein Beratungsinstrument mit prozeduralem Charakter, das sich an Akteure wendet, die unter bestimmten systemischen und institutionellen Bedingungen technikbezogene Entscheidungen zur Vermeidung von negativen und zur Förderung von positiven Folgen zu treffen haben. Unter bestimmten politischen Verhältnissen kann aus dem Beratungs- auch ein Entscheidungsinstrument werden, wie z. B. im Falle der dänischen Konsensuskonferenz. Aber es gibt selbst in diesem Falle Herren und Damen des Verfahrens, die sich auf das Instrument und wie es eingesetzt werden soll, einigen müssen. Solange TA keine rechtliche Form annimmt, bleibt sie ein Instrument, eine Wissensressource, von der Entscheidungsträger wie Advocacy-Akteure Gebrauch machen können oder eben auch nicht.

\section{Literatur}

Beck, S. 2009, Das Klimaexperiment und der IPCC: Schnittstellen zwischen Wissenschaft und Politik in den internationalen Beziehungen. Marburg: Metropolis.

Beck, S. 2011, Zwischen Entpolitisierung von Politik und Politisierung von Wissenschaft: Die wissenschaftliche Stellvertreterdebatte um Klimapolitik, in Schüttemeyer, Suzanne S. (Hg.): Politik im Klimawandel: Keine Macht für gerechte Lösungen? Baden-Baden: Nomos, 239-258.

Benz, A., u. a. 2007, Einleitung, in Benz, Arthur, u. a. (Hg.): Handbuch Governance: Theoretische Grundlagen und empirische Anwendungsfelder. Wiesbaden: VS, Verl. für Sozialwiss., 9-25.

Bolin, B. 2007, A history of the science and politics of climate change: The role of the Intergovernmental Panel on Climate Change. Cambridge: Cambridge University Press.

Bröchler, S. 2008, Governance im Lichte der sozialwissenschaftlichen Technikforschung, in Bröchler, Stephan und Lauth, Hans-Joachim (Hg.): Politikwissenschaftliche Perspektiven. Wiesbaden: VS, Verl. für Sozialwiss., 45-56.

Bröchler, S. 2010, Technikfolgenabschätzung und Technology Governance: Steuerung und Koordination in der Governance-Perspektive, in Aichholzer, Georg (Hg.): Technology Governance: Der Beitrag der Technikfolgenabschätzung. Berlin: Edition Sigma, 63-83.

Bröchler, Stephan und Schützeichel, Rainer (Hg.) 2008, Politikberatung. Stuttgart: Lucius \& Lucius.

Dolata, U. 2011, Wandel durch Technik: Eine Theorie soziotechnischer Transformation. Frankfurt am Main: Campus.

Ende, Jan van den und Kemp, René (1999): Technological transformations in history: how the computer regime grew out of existing computing regimes. Research Policy, 28 (8), 833-851.

Fach, W. und Simonis, G. 2000, Die Welt des Autors, Zeitschrift für Internationale Beziehungen $7(2), 385-398$. 
Geels, F. W. und Schot, J. 2007, Typology of Sociotechnical transition pathways, Research Policy 36(3), 399-417.

Grunwald, A. 2010, Technikfolgenabschätzung - eine Einführung. 2. Aufl. Berlin: Ed. Sigma.

Kuhlmann, S. 2010, TA als Tanz: Zur Governance technologischer Innovation: Neue Aufgaben des Technology Assessment, in Aichholzer, G., Bora, A., Bröchler, S., Decker, M., Latzer, M. (Hg.): Technology Governance: Der Beitrag der Technikfolgenabschätzung. Berlin: Edition Sigma, 41-57.

Lingner, S. 2010, Rationale Technikfolgenbeurteilung: Ein deliberativer TA-Ansatz im Kontext von „Technology Governance“, in Aichholzer, Georg (Hg.): Technology Governance: Der Beitrag der Technikfolgenabschätzung. Berlin: Edition Sigma, 93-111.

Luhmann, N. 2008, Ökologische Kommunikation: Kann die moderne Gesellschaft sich auf ökologische Gefährdungen einstellen? 5. Aufl. Wiesbaden: VS Verlag für Sozialwissenschaften.

Rip, A. und Kemp, R., 'Technological Change', in Rayner, Steve und Malone, Elizabeth L. (Hg.): Human Choice and Climate Change. Columbus, OH: Batelle Press, 327-399.

Simonis, G. 2001, Die TA-Landschaft in Deutschland - Potenziale reflexiver Techniksteuerung, in Simonis, Georg (Hg.): Politik und Technik: Analysen zum Verhältnis von technologischem, politischem und staatlichem Wandel am Anfang des 21. Jahrhunderts. Wiesbaden: Westdt. Verl, 425-456.

Simonis, Georg (Hg.) 2001, Politik und Technik: Analysen zum Verhältnis von technologischem, politischem und staatlichem Wandel am Anfang des 21. Jahrhunderts. 1. Aufl. Wiesbaden: Westdt. Verl.

Simonis, G. 2011, Das Transformationspotenzial des Emissionshandels - analysiert am Beispiel der Waxman-Markey-Vorlage im US-Kongress, in Schüttemeyer, Suzanne S. (Hg.): Politik im Klimawandel: Keine Macht für gerechte Lösungen? Baden-Baden: Nomos, 159-193.

Streeck, W. 2009, Re-forming capitalism: Institutional change in the German political economy. Oxford, UK: Oxford University Press.

Streeck, W. und Thelen, K. A. 2005, Introduction:Institutional Change in Advanced Political Economies, in Streeck, Wolfgang und Thelen, Kathleen A. (Hg.): Beyond continuity: Institutional change in advanced political economies. Oxford;, New York: Oxford University Press, 1-39.

Voss, Jan-Peter, Bauknecht, Dierk und Kemp, René (Hg.) 2006, Reflexive governance for sustainable development. Cheltenham, Glos, UK; Northampton, MA: Edward Elgar.

Weingart, P. und Lentsch, J. 2008, Wissen, Beraten, Entscheiden: Form und Funktion wissenschaftlicher Politikberatung in Deutschland. 1. Aufl. Weilerswist: Velbrück. 



\title{
Trotz aller Differenzen \\ Theoretische und empirische \\ Perspektiven auf das Hummel-Paradox \\ der (Technology-)Governance
}

\section{Marc Mölders}

Institut für Wissenschafts- und Technikforschung (IWT), Universität Bielefeld

\section{Keywords}

Governance, Verhandlungssysteme, funktionale Differenzierung, Hummel-Paradox, Single European Sky

\begin{abstract}
Das Hummel-Paradox der Governance-Forschung bezeichnet den Umstand, dass es in den für Governance charakteristischen Verhandlungssystemen trotz einer Vielzahl heteogener Mitspieler zu produktiven Ergebnissen kommen kann. Wie die Gesetze der Aerodynamik den Hummelflug nur scheinbar verhindern, so trifft Analoges auf die Bedingungen funktionaler Differenzierung für Governance zu: Der Eigensinn der Mitspieler verhindert grenzüberschreitendes Gelingen nicht.Um gelingende Verhandlungssysteme zu qualifizieren, werden im Beitrag eine operative und eine integrative Leistungsdimension eingeführt und an einem Beispiel belegt. Daraufhin werden Ansätze eingeführt, die zu erklären versuchen, warum Eigensinn diesenErfolg nicht blockiert. Am aktuellen Fall des „Single European Sky“ wird abschließend gezeigt, inwiefern die zuvor skizzierten Theorien einer empirischen Konkretisierung bedürfen.
\end{abstract}

\section{Despite all Differences: Theoretical and Empirical Perspectives on the Bumblebee Paradox of (Technology-) Governance}

Keywords: Governance, negotiation systems, functional differentiation, bumblebee paradox, Single European Sky

\footnotetext{
Abstract: The bumblebee paradox of governance refers to the puzzle that negotiation systems can lead to productive, cross-border results although its participants remain idiosyncratic. Aerodynamic laws do not prevent bumblebees from flying; the same can be said about the conditions of functional differentiation with regard to governance: There are successful negotiation systems despite its participants being idiosyncratic. To qualify successful negotiation systems, an operative and an integrative dimension of performance are introduced. The benefit of this distinction is demonstrated by an example. This is followed by a selection of approaches trying to explain why idiosyncrasy does not prevent integrative and operative successes. The discussion of the recent case "Single European Sky" will show that theoretical approaches need a sharper focus in order to make empirically adequate statements.
} 


\section{Einleitung}

Bis zum Ende des 20. Jahrhunderts konnte die Legende, Hummeln könnten den Gesetzen der Aerodynamik zufolge nicht fliegen, wissenschaftlich nicht widerlegt werden. Dass sie fliegen können, war für jedermann ersichtlich, das „Wie“ bzw. das „Warum-trotzdem“ war das Rätsel.

Nun sind für Governance, zumindest wenn man sich an das engere Begriffsverständnis von Stephan Bröchler (Bröchler 2007: 414) hält, Verhandlungssysteme charakteristisch, in denen sich Politik mit anderen betroffenen gesellschaftlichen Bereichen vernetzt. Hier würden dann die Bedingungen funktionaler Differenzierung bzw. von Polykontexturalität die Rolle der Gesetze der Aerodynamik übernehmen: Eigentlich müssten die Geschlossenheit und der Eigensinn sozialer Systeme es verhindern, dass solche Intersystem-Verhandlungssysteme (Teubner 1989: 80) gelingen können. Doch zumindest der soziologische Beobachter kann unmittelbar sehen, dass es gelingende Verhandlungssysteme ebenso gibt, wie den Hummelflug. Es gibt Erklärungen für dieses Warum-Trotzdem, von denen einige theoretisch überzeugend sind, forschungspraktisch aber durchweg von einer Annahme ausgehen, die ich im Folgenden hinterfrage: Sie wissen immer schon, wonach (genauer: nach welchen Differenzen) sie suchen.

Doch bevor dies diskutiert werden kann, sind einige Schritte voranzustellen: Der erste Abschnitt wird zu klären haben, was gelingende Verhandlungssysteme überhaupt kennzeichnet. Um meinen Vorschlag einer operativen und einer integrativen Leistungsdimension zu plausibilisieren und zu veranschaulichen, wird mit der sogenannten „von Wedel-Kommission“ (zur Organisation des gesundheitlichen Verbraucherschutzes) ein empirisches Erfolgsbeispiel skizziert.

Ist damit ein Teil des Hummel-Paradoxes belegt („Es gibt trotz der Bedingungen funktionaler Differenzierung erfolgreiche Intersystem-Verhandlungssysteme“), werden im zweiten Abschnitt einige Erklärungsansätze für ein Darum-Trotzdem erörtert. Dabei wird sich zeigen, dass nur durch die Umstellung auf handlungsfähige (korporative) Akteure allein dem Paradox nicht beizukommen ist. Plausible Erklärungen finden sich bemerkenswerterweise bei einem akteurzentierten Ansatz, der gleichwohl davon ausgeht, systemfremde Akteure müssten aneinander vorbeireden, wie auch bei einem kommunikationsbasierten Konzept, das die Möglichkeit gemeinsamer Leitunterscheidungen vorsieht.

Der dritte und letzte Abschnitt zeigt dann, dass trotz dieser unterschiedlichen Grundannahmen relevante Gemeinsamkeiten auszumachen sind. Analytisch orientieren sich diese Ansätze auf der Differenzierungsebene der Teil- bzw. Funktionssysteme. Die Angemessenheit dieses Ausgangspunktes wird dann an einem Fallbeispiel in Zweifel gezogen. Das Verhandlungssystem „Single European Sky“ - eine EU-Initiative zur technischen, operativen wie rechtlichen Vereinheitlichung des europäischen Luftraums - weist zentrale Teilnehmer auf, die sich nicht der Ebene der Funktionssysteme zuordnen lassen. Hieraus wird der Schluss gezogen, dass nicht in jedem Fall vorab feststeht, wonach zu suchen ist. Eine kurze Erörterung möglicher theoretischer, empirischer wie praktischer Konsequenzen beschließt den vorliegenden Beitrag. 


\section{Gelingende Governance: Zur Unterscheidung einer operativen und einer integrativen Leistungsdimension am Beispiel der „von Wedel-Kommission“}

Die aufgeworfene Problemstellung erfordert zunächst einmal zwei Klärungen:

1. Was heißt überhaupt gelingende Governance?

2. Gibt es solche Erfolgsfälle?

Um den Erfolg von Verhandlungssystemen zu qualifizieren, schlage ich die analytische Unterscheidung einer operativen und einer integrativen Leistungsdimension vor. Verhandlungssysteme werden mit dem Ziel eingerichtet, eine kollektiv verbindliche Entscheidung herbeizuführen oder zumindest vorzubereiten. Aus diesem recht breiten Verständnis fallen etwa partizipative Verfahren ohne konkretes Ziel in diesem Sinne heraus; an sie müssten andere Kriterien angelegt werden (vgl. Abels/Mölders 2007).

Die operative Leistungsdimension fragt danach, ob ein Verhandlungssystem zu einem Resultat führt, das im Folgenden auch verwendet wird. Ob die unterschiedlichen Beteiligten in ihrem je eigenen Sinne an dieses Resultat anschließen können, ist Kernfrage der integrativen Dimension.

Wenn schlussendlich jeder Beteiligte eigensinnig anschließt, kann man sich wundern, warum ausgerechnet dieser Umstand, ,integrativ“ zu nennen sein soll. Wie im nächsten Abschnitt gezeigt wird, gehen relevante Teile der auch gesellschaftstheoretisch argumentierenden Governance-Forschung davon aus, dass die Diagnose funktionaler Differenzierung gar nichts anderes zulässt, als dass aneinander vorbeigeredet werden muss, hieraus aber nicht zwingend der Schluss gezogen werden muss, dass damit jedwede Steuerungsabsicht ins Leere laufen muss. Das gemeinsame Moment liegt im Resultat selbst, schließlich ist es etwa ein technischer Standard, der für die am Verhandlungssystem Beteiligten unterschiedliche, aber untereinander kompatible Anschlüsse ermöglicht: politische Entscheidungen, Zahlungsoperationen, Rechtsakte usw. (vgl. Teubner 1999: 13f.).

Ein Beispiel vermag diese analytische Trennung auf empirische Füße zu stellen. Die sogenannte von Wedel-Kommission wurde Ende des Jahres 2000 vom seinerzeit amtierenden Bundeskanzler mit einer Schwachstellenanalyse zur Lebensmittelsicherheit beauftragt. Besetzt war dieses Netzwerk mit Experten und Vertretern aus Wissenschaft, Wirtschaft, Verbraucherschutz und Landwirtschaft, die ihre Analyse wiederum mit der Politik abstimmen mussten. Folglich gibt es gute Gründe, von einem Intersystem-Verhandlungssystem auszugehen. Kernfrage war, ob die bestehenden Institutionen den neuen Problemen des gesundheitlichen Verbraucherschutzes (Stichwort: BSE) angemessen seien. Die Kommission schloss in ihrem Gutachten bekanntermaßen, dass eine Reform des Landwirtschaftsministeriums zu empfehlen sei (von Wedel 2001). Dieser Empfehlung folgte die Politik, das Ministerium wurde weitreichend reformiert, der Verbraucherschutz priorisiert. Das Resultat des Verhandlungssystems wurde also verwendet (operativ). Die hieran Beteiligten konnten in ihrem je eigenen Sinne anschließen, konnten Mehrwerte generieren, die ohne Partizipation am Verhandlungssystem unerreichbar gewesen wären (integrativ): Die Politik konnte endlich mit ihren Mitteln auf die BSE-Krise reagieren, der sie sich bis dato ohnmächtig gegenüber sah. Die Wirtschaft gewann einen Anhaltspunkt für die Wiederbelebung des Rindfleischkonsums, der Verbraucherschutz hatte vor allem institutionelles Kapital eingetragen, schließlich war ihm ein ministerielles Ressort gewidmet worden usw. (vgl. Böschen et al. 2003). 
Man kann die Leistungsdimensionen auch aufeinander beziehen: Je stärker die integrative Dimension Berücksichtigung findet, desto höher ist auch die Wahrscheinlichkeit eines operativen Erfolgs. Offensichtlich können Verhandlungssysteme also sowohl operativ als auch integrativ leistungsfähig sein. Ebendies war ja mit dem Hummel-Paradox der Governance-Forschung angesprochen: Die Beobachtung erfolgreicher Intersystem-Verhandlungssysteme, obwohl die Bedingungen funktionaler Differenzierung (Polykontexturalität bzw. systemischer Eigensinn) dies eigentlich nicht zulassen dürften.

Schon gegen diese Art der Fragestellung bzw. Problembeschreibung sind vielfach Einwände erhoben worden, allen voran: Man habe es mit einer erledigten Debatte (systemtheoretische Steuerungskonzepte) zu tun, deren Probleme hausgemacht waren und sich durch eine Umstellung auf (korporative) Akteure und Handlung, anstelle von System und Kommunikation, lösen. Ansätze zur Lösung des Hummel-Paradoxes gibt es einige - und dies über unterschiedliche Grundbegriffe und fokussierte Einheiten (wie Akteure, Organisationen, Teilsysteme etc.) hinweg. ${ }^{1}$ Im nächsten Abschnitt werde ich zunächst zeigen, dass auch eine Umstellung auf Akteure das Problem nicht löst, sondern eher verschiebt, wie aber trotzdem die Abstimmung systemisch distinkter Akteure auch handlungstheoretisch erklärt werden kann. Überraschenderweise insistiert dieser Ansatz (Schimank) darauf, dass in Intersystem-Verhandlungssystemen aneinander vorbeigeredet werden muss. Nicht minder überraschend ist, dass ein kommunikationsbasierter, systemtheoretischer Ansatz (Hutter) hingegen von der Prämisse ausgeht, dass verstetigte Interaktionen zu gemeinsamen Leitunterscheidungen führen können.

\section{Theoretische Entfaltungen des Hummel-Paradoxes: Differenzen und Gemeinsamkeiten}

Beim oben genannten Einwand denkt man beinahe automatisch an den akteurzentriertenInstitutionalismus von Renate Mayntz und Fritz Scharpf. Beide haben ja gerade darauf insistiert, an der gesellschaftstheoretischen Diagnose funktionaler Differenzierung festzuhalten, den empirischen Fokus aber auf korporative Akteure in Verhandlungssystemen zu richten (etwa in: Mayntz/Scharpf 2005).

Wesentlich für diese Argumentation ist die Handlungsfähigkeit von Organisationen. Allerdings wird zwischen Handlungsfähigkeit und ,systemrationalen Lösungen“ keine Kausalität unterstellt. Solche Lösungen, so schwierig sie im Einzelfall zu beurteilen sein mögen, sind dann gegeben, wenn es um sachlich adäquate Entscheidungen jenseits individuell unterschiedlicher Strategien geht. Um es in Renate Mayntz' eigenen Worten zu sagen, systemrationale Lösungen werden oftmals dadurch verunmöglicht, dass auch Organisationen ,selbstsüchtig und kurzsichtig handeln“ können (Mayntz 1993: 45). Damit ist ein interessantes Problem angesprochen: Eigensinn endet folglich nicht an den Grenzen von Funktionssystemen. Auch Organisationen verfügen über Informationsverarbeitungen eigener Art. Mayntz begnügt sich nicht mit dieser Problemdiagnose, vielmehr sieht sie einen Ausweg in Richtung einer Überwindung organisationalen Eigensinns, ihrer Selbstsucht und Kurzsicht.

1 Eine diesbezügliche Übersicht wird in einem Beitrag von mir in Bora et al. (2012) erscheinen. 
Ihre Erklärung benötigt eine bestimmte Sorte von Akteuren: die „,cosmopolitans“. Diese sind nicht im engeren Sinne weisungsgebunden an die „Politik des Hauses“, sondern orientieren ihre Handlungen stärker an professionellen Standards, etwa den Regeln ihrer scientific community. Im Gegensatz dazu stünden mit den ,locals“ Akteure der Managementebene, die den Eigeninteressen der Organisation in deutlich höherem Maße verpflichtet seien. Treffen in Verhandlungssystemen nun vornehmlich Kosmopoliten aufeinander, bestünde die realistische Chance auf eine sachlich angemessene Entscheidungsfindung. Beispiele für solche Konstellationen sieht Mayntz in Verfahren technischer Standardisierung. Gerade hier könne man empirisch beobachten, dass es im Kern um technisch optimale Lösungen ginge und dementsprechend nur technische Argumente zählten (vgl. ebd. 52f.).

Dann aber gibt es das Problem der Polykontexturalität schlicht nicht mehr. Was man hier beobachtet, ist nicht die Überwindung intersystemischen oder auch interorganisationalen Eigensinns. Statt der Abstimmung unterschiedlicher und untereinander inkompatibler Sichtweisen existiert in diesen Fällen mit der technischen eine einheitliche Sicht der Dinge. Technische Argumente fungieren als Amtssprache solcher Verhandlungssysteme.

Renate Mayntz und Fritz Scharpf haben vielerlei Fälle beschrieben, die zwar nicht von systemrationalen Lösungen gekennzeichnet waren, allerdings insofern zu einem erfolgreichen Ende kamen, als Kompromissbildungen zu verzeichnen waren. An dieser Stelle zeigt sich der Gewinn der in diesem Abschnitt eingeführten Unterscheidung: Operativ sind solche Fälle unzweifelhaft als gelungen zu qualifizieren. In integrativer Hinsicht erscheint zumindest fraglich, ob an die gefundenen Kompromisse tatsächlich unterschiedlich anzuschließen war, oder ob es sich eher um einseitige Anpassungen handelte.

Eine Umstellung auf handlungsfähige Akteure allein entledigt jedenfalls nicht vom Problem der Polykontexturalität. Uwe Schimank hat, auf Basis der Überlegungen des akteurzentrierten Institutionalismus, einen eigenen Lösungsvorschlag für das Hummel-Paradox anzubieten. Wie oben bereits angedeutet, wird Polykontexturalität als Problem unmittelbar in das Modell integriert. Schimanks Ausgangspunkt ist, dass Akteure unterschiedlicher Funktionssysteme aneinander vorbeireden müssen, was jedoch nicht hieße, dass sie einander nicht mehr oder weniger gut verstehen könnten (vgl. Schimank 1992: 254).

Eine unterschiedliche funktionssystemische Zugehörigkeit geht mit unterschiedlichen Interessen einher. Zwar nehmen sich diese in konkreten Fällen unterschiedlich aus - ein Wissenschaftler hat andere Interessen als ein Politiker usw. -, dennoch lassen sich, so Schimank, reflexive Interessen gewissermaßen überall finden, weil es sich dabei um solche handelt, ,die sich auf die generellen Bedingungen der Möglichkeit der Realisierung spezifischer substantieller Interessen“ beziehen (ebd.: 264). Konkret geht es dabei um (1) die Ausdehnung der Reichweite der Interessenrealisierung, (2) das Erlangen bzw. Verteidigen von Dominanz in einer Interessensphäre und (3) die Erweiterung der Kontrolle über die eigene Interessenrealisierung bzw. Entscheidungsautonomie (ebd.).

Um spezifische Interessenkonsense zu erreichen, ist mehr als eine Unterstellung reflexiver Interessen nicht notwendig. Die konkrete Operationsweise anderer Kontexte muss dabei nicht nachvollzogen werden. Politiker müssen nicht auf dem neuesten Stand der Forschung sein, um davon ausgehen zu können, dass eine Forschungseinrichtung ein Interesse daran haben dürfte, auf ihrem Fachgebiet eine Spitzenposition zu erlangen und hierfür Geld benötigt. Empirisch hat Schimank dies am Beispiel der frühen bundesdeutschen Kernforschung bzw. Kernforschungspolitik illustriert. Auf empirische Implikationen komme ich abschließend zurück.

Reflexive Interessen, das ist der entscheidende Zug für das Thema dieses Beitrags, kompensieren das Fehlen einer gemeinsamen Sprache. Um welche Interessensphäre es dabei konkret geht, orientiert sich, wie bereits ersichtlich wurde, an den Logiken der Funktionssysteme. Schimank erklärt die Reproduktion ebendieser Logiken damit, dass sie schon so sehr verinnerlicht seien, dass die Ak- 
teure immer schon so handeln, als ob für PolitikerInnen nur die (Wieder-)Wahl zählt, für Wirtschaftsvertreter die Aussicht auf Zahlungen, es für Wissenschaftler stets um ideale Voraussetzungen für die Erkenntnisproduktion geht usw. Auf eine Formel gebracht: Die Bedingungen funktionaler Differenzierung sind zu einer self-fulfilling prophecy bzw. zu verdinglichten Akteurfiktionen geworden (vgl. Schimank 2009: 200ff.; 2011).

Natürlich gibt es auch näher an der systemtheoretischen Vorlage orientierte Erklärungsansätze. Dabei ließe sich etwa an Helmut Willkes (1992; 1997) Konzept einer dezentralen Kontextsteuerung oder auch an Gunther Teubners klassisches Modell des Ultrazyklus (1998; 1999) denken. Auch wenn diese Konzepte auf der Basis einer Kommunikationstheorie argumentieren, teilen sie mit Schimanks Akteurfiktionen, dass aneinander vorbeigeredet werden muss, dies aber nicht ausschließt, etwas zu produzieren, von dem alle in ihrem Sinne profitieren.

In dieser Hinsicht markiert der Vorschlag der Konversationskreise Michael Hutters (1989) eine bemerkenswerte Ausnahme. Im Gegensatz zu ansonsten flüchtigen Interaktionssystemen werden bei diesen Zusammentreffen Gesprächsorte, -zeitpunkte und -teilnehmende zunehmend standardisiert. Wenn einerseits Kommunikationen dem Kreis und nicht seinen einzelnen Elementen zugerechnet werden und andererseits eine interne Verdichtung derart auszumachen ist, dass neben den fokussierten Themen auch über sich selbst gesprochen wird, hat sich ein neuer Konversationskreis gebildet. $\mathrm{Ob}$ es sich dabei um Arbeitsgemeinschaften oder Vereinigungen unterschiedlicher Art handelt, gemein ist diesem Typus, dass sie mit interaktiven Zusammentreffen beginnen, dann aber der Eindruck erwächst, man könne es bei der für Interaktionssysteme typischen Vergänglichkeit nicht belassen (vgl. Hutter 1989: 99f.).

Polykontexturalität kann geradezu als das Kernproblem angesehen werden, auf das das Theorem der Konversationskreise antwortet. Die Annahme ist, dass im Laufe der oben beschriebenen Verstetigung ,soziale Formen“ entstehen, durch die Mitteilungen beispielsweise für wirtschaftliche und rechtliche Teilnehmende ,,besser formuliert und besser verständlich werden. [...] Soziale Systeme, die auf die Durchführung von Konversationen spezialisiert sind, werden wir Konversationskreise nennen“ (ebd.: 94). Konversationskreise kennzeichnet, dass sie mehrere Leitunterscheidungen präsent halten, also etwa Mitteilungen produzieren, die sich sowohl am Wert- (Wirtschaft) als auch am Gerechtigkeitskriterium (Recht) orientieren (s. Abb. 1). Gemessen am systemtheoretischen Postulat operativer Geschlossenheit mutet es geradezu revolutionär an, dass Hutter sogar die Ausbildung einer gemeinsamen, gleichsam instabilen Leitunterscheidung ${ }^{2}$ vorsieht (vgl. ebd.: 136f.). Eine Amtssprache in Mayntz' Sinne ist damit gerade nicht angesprochen; die rechtlichen und wirtschaftlichen Mitteilungsströme bleiben vollwertig differenziert. Die gemeinsamen Leitunterscheidungen sind als solche überhaupt nur zu erkennen, wenn man sie als anschlussermöglichend für Unterschiedliches konzipiert.

Die Leitunterscheidungen bei Hutter, die Akteurfiktionen bei Schimank, die Sinnprodukte bei Teubner, sie alle eint eines: Man kennt sie bereits vorab und kann im empirischen Material dann gewissermaßen nach ihnen suchen. Weiterhin fällt auf, dass sie sich ebenso alle an der Ebene der Teiloder Funktionssysteme orientieren. Schaut man sich Verhandlungssysteme allerdings empirisch an, wird man feststellen, dass hieran in aller Regel Organisationen beteiligt sind. Schimank hat dieses Problem vor Augen, löst es allerdings unmittelbar mit der Annahme, dass Organisationen im Normalfall nur einem Funktionssystem angehören (vgl. Schimank 2009: 198).

2 In Hutters Fall des Arzneimittelpatentrechts bildeten „Rechte auf Handlungen“ eine gemeinsame Grenze der Wirtschaft mit dem Recht (vgl. ebd.:138). 


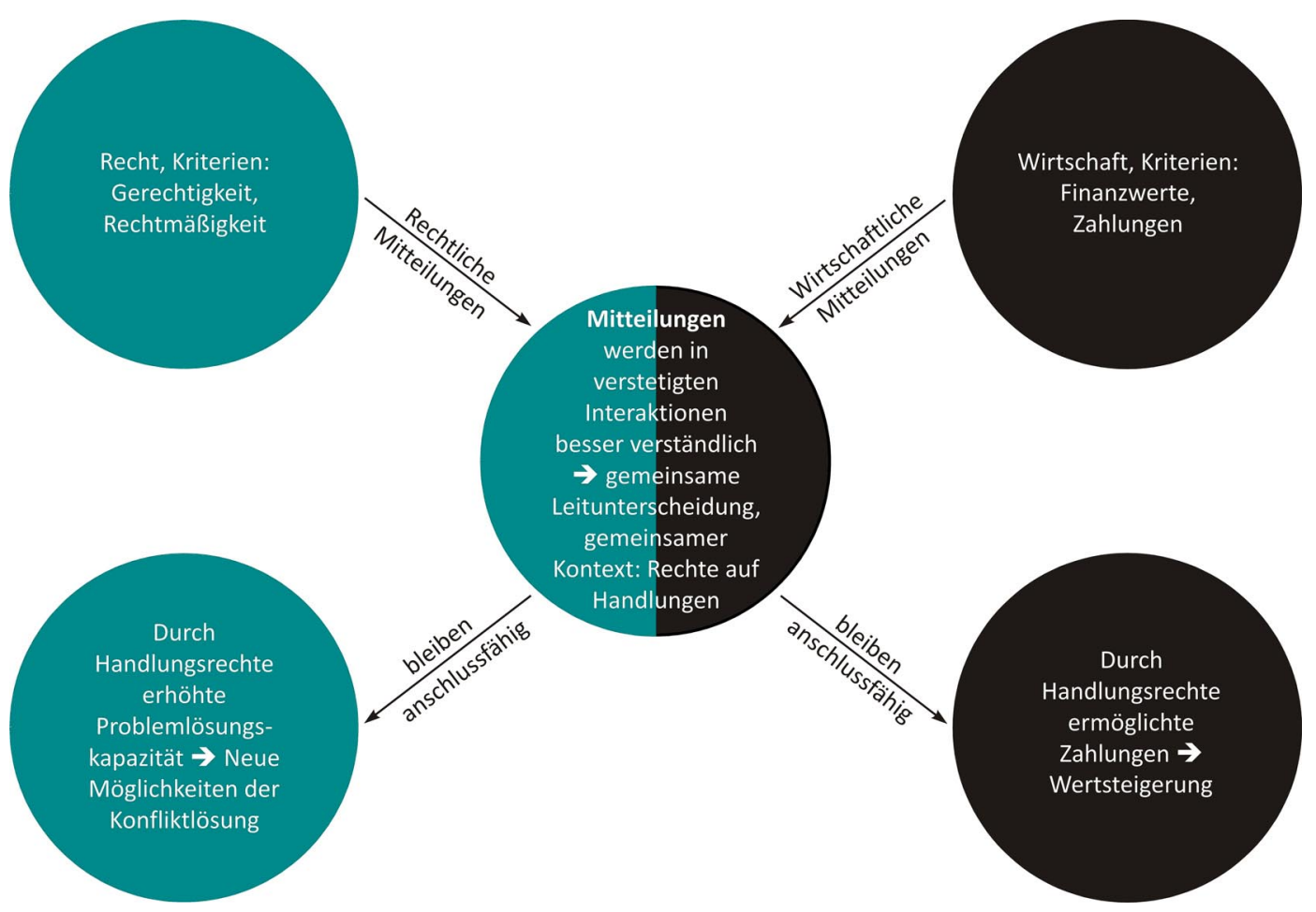

Abbildung 1: Konversationskreise

Erst die entgegengesetzte Annahme, Organisationen verfügten über Informationsverarbeitungen, die nicht umstandslos einem Funktionssystem zuzuordnen sind, nährt die Zweifel an der empirischen Angemessenheit dieser Differenzierungsebene. Dass es sich dabei nicht nur um ein theoretisches, sondern vielmehr um ein empirisches und praktisches Problem handelt, wird der folgende Abschnitt an einem Fallbeispiel verdeutlichen.

\section{Empirische Perspektiven auf das Hummel-Paradox: Der Fall des ,Single European Sky“}

Erste Bemühungen der Europäischen Union um einen vereinheitlichten europäischen Luftraum sind seit Ende der 1990er Jahre zu verzeichnen. Dass es einer Vereinheitlichung, Harmonisierung und Synchronisierung der Dienste im Rahmen des europäischen Flugverkehrsmanagements bedarf, wird gemeinhin kaum bezweifelt. So endeten etwa bis zu Beginn dieses Jahres die Zuständigkeiten der Flugsicherungen an den Grenzen der Nationalstaaten, die bekanntermaßen eng sind in Europa. Unterschiedliche technische, operative und rechtliche Standards regeln den europäischen Flugverkehr, was unmittelbar ersichtlich mit einem enormen Koordinierungsaufwand auf einem Gebiet einhergeht, in dem schon kleine Fehler fatale Konsequenzen nach sich ziehen können. 
2007 gründete die EU-Kommission gemeinsam mit Eurocontrol, der europäischen Organisation für die Sicherheit der Luftnavigation, das Single European Sky ATM Research Programme Joint Undertaking (SESAR JU bzw. SJU). SESAR kennzeichnet das Prinzip der Allinklusion: Alle am Luftverkehr beteiligten Parteien sollen gemeinsam über die zur Disposition stehenden Standards befinden und kollaborieren. Somit sind nunmehr zahlreiche Parteien Teil des SJU, sei es als Mitglied, als Stakeholder, als Teil eingesetzter Expertenkommissionen, durch Anhörungen usw. In ebenso zahlreichen Workpackages, Projekten und Sub-Projekten kommen Vertreter von Flugsicherungen, Gewerkschaften, dem Militär, europäischen und nationalstaatlichen Regulierern, Fluggesellschaften, Forschung, Industrie u.v.m. zusammen.

Schon aufgrund dieses Allinklusionsprinzips ist SESAR für Fragen der Abstimmung in Intersystem-Verhandlungssystemen ein besonders vielversprechender Kandidat. Für das oben aufgeworfene Problem ist insbesondere die Rolle der Organisation Eurocontrol von einigem Interesse. Sie ist Gründungsmitglied der ausführenden Instanz SESAR JU, vor allem aber ist Eurocontrol als einzige Partei an ausnahmslos allen Workpackages beteiligt. Ein Blick in programmatische Organisationsdokumente zeigt, dass Eurocontrol nicht umstandslos einem Funktionssystem zurechenbar ist. Direktoratsmitglied Bernard Miaillier (2010) führtaus: „Because of our neutral position as a European organisation that is not a manufacturer, aircraft user or competitor with other participants, we can bring benefits, especially when prioritising different solutions, by providing impartial assessments."

Die Leistungsfähigkeit wird hier durch die nicht-selbstverständliche Verknüpfung der Eigenschaften „europäisch“ und „,neutral“ begründet. Bemerkenswert ist gerade die Betonung, weder politisch noch wirtschaftlich interessiert zu sein: „EUROCONTROL'sunique strengt his being impartial, with no vested national or commercial interest“ (EUROCONTROL 2010: 7). Ohne hierauf näher eingehen zu müssen und ohne dem Verdacht ausgesetzt zu sein, fahrlässig einer Selbstbeschreibung auf den Leim zu gehen, sollten zwei Aspekte deutlich geworden sein: 1. Dass Eurocontrol eine besonders gewichtige Rolle in diesem Verhandlungssystem einnimmt. 2. Dass sich diese Rolle nicht durch eine Subsumption unter eine Funktionssystemlogik fassen lässt.

Als operatives wie technologisches Herzstück des SES wird die Entwicklung des System Wide Information Management (SWIM) gesehen. Mit diesem allen Beteiligten zugänglichen Intranet sollen alle relevanten Informationen ständig verfügbar sein und unmittelbar aktualisiert werden. Vielleicht also behält am Ende, zumindest mit Blick auf diesen Fall, Renate Mayntz doch Recht, und technische Expertise entpuppt sich als Amtssprache dieses Verhandlungssystems. Mag dies mit Blick auf Eurocontrol noch für möglich gehalten werden, macht die weitere Teilnehmerliste (Industrie, Militär, Flugsicherer etc.) die Annahme plausibler, dass es sich um einen Fall handelt, in dem die Teilnehmenden signifikant unterschiedliche Informationsverarbeitungen aufweisen.

In theoretischer, empirischer und schließlich praktischer Hinsicht zeitigt dieser auf den ersten Blick vermeintlich unscheinbare Fall einige Konsequenzen: Differenzierungstheoretisch muss es für möglich gehalten werden, dass es zumindest für konkrete Ausschnitte sozialer Wirklichkeit (wie etwa Verhandlungssysteme) relevante Differenzierungsformen gibt, deren Leitunterscheidungen, Deutungsmuster, Wertorientierungen usw. nicht aus theoretischen Annahmen (allen voran: den Funktionssystemen) abzuleiten sind. Daraus ergibt sich die empirische Konsequenz, voreiligen Subsumptionen skeptisch gegenüber zu stehen und die Leitunterscheidungen kritischer Fälle etwa mithilfe von Dokumentenanalysen und/oder Interviews offen zu rekonstruieren. Die praktische Folge hängt unmittelbar mit den theoretischen und empirischen Konsequenzen zusammen. Wenn schon Differenzierungstheoretiker eine Organisation wie Eurocontrol nicht einzuordnen wissen, wie machen es dann diejenigen, die mit ihr am Verhandlungstisch sitzen? Michael Hutter (Hutter 1989: 138) nimmt an, dass systemfremde Interaktionsteilnehmer sich intern ein Bild der anderen machen, ,das ihren Bedürfnissen genügt, und die Kunst besteht darin, mit dem geringsten Aufwand an Detailstrukturierung dieses Images eine erwünschte Leistung zu erzielen.“ 
Insofern kann man sich fragen, ob die analytisch plausibilisierte Existenz nicht-subsumierbarer Differenzierungsformen empirisch nicht nur möglich, sondern auch nötig ist: Was, wenn trotzdem reflexive Interessen entlang der Funktionslogiken unterstellt werden und spezifische Interessenkonsense entstehen (Schimank) oder sich dessen ebenso ungeachtet gemeinsame Leitunterscheidungen (Hutter) herausbilden?

Doch auch dies ist nur empirisch zu rekonstruieren und würde, sofern Orientierungen an den Codes der Funktionssysteme nicht einmal als self-fulfillingprophecy nachzuweisen wären, ein theoretisches Nachjustieren bedeuten. Das abschließende Plädoyer lautet davon unabhängig: Die hier aufgeworfenen Fragen empirisch zu beantworten und hieran ggf. die Theorie auszurichten - und nicht umgekehrt.

Technology Governance meint das Zusammenwirken zivilgesellschaftlicher (wie Unternehmen, Verbände, Initiativen, Wissenschaft) und staatlicher Akteure bei der Entwicklung und Nutzung technischer Innovationen. Insofern ist die Lösung des hier thematisierten Hummel-Paradoxons von größtem Interesse, denn es geht hierbei ja gerade um Formen des Zusammenwirkens, die trotz aller Differenzen zu erfolgreichen Resultaten führen.

Der vorliegende Beitrag hat zunächst mit der Unterscheidung einer operativen von einer integrativen Leistungsdimension einen Vorschlag unterbreitet, wie gelingende Technology Governance zu qualifizieren ist.Das Modell der Konversationskreise hat gezeigt, dass sachlich angemessene Problemlösungen durchaus mit Resultaten einhergehen können, die in sozialer Hinsicht unterschiedliche Anschlüsse zulassen - hierauf zielen die operative und die integrative Leistungsdimension.

Schließlich kommt mit der zeitlichen eine letzte Komponente ins Spiel: Gemeinsamkeiten - hier: gemeinsame Leitunterscheidungen bzw. Kontexte - brauchen Zeit.Gerade mit Blick auf technische Innovationen ist Zeit besonders knapp, weshalb Ansätze wie Constructive TA (Rip 2006) oder Anticipatory Governance (Barben et al. 2008) für ein möglichst frühzeitiges Zusammenwirken plädieren. Doch für Konversationskreise, so Hutter (1989: 94), sei gerade nicht naheliegend, dass sie „gemacht oder gegründet“ werden. Sie entstehen erst, wenn das „operative Geschäft“ die Beteiligten zusammenführt. Können bereits gelungene Fälle aufgezeigt und erklärt werden, erleichtert dies Technology Governance die schwierige Aufgabe, potentiellen Beteiligten zu verdeutlichen, dass es auch in ihrem Sinne sein kann, zu (inter)agieren, bevor das Tagesgeschäft sie dazu drängt.

\section{Literatur}

Abels, G., Mölders, M., 2007, Meeting of Minds. Kritische Beobachtungen zu Form und Funktion der ersten europäischen Bürgerkonferenz, in: Bora, A., Bröchler, St. und Decker, M. (Hg.): Technology Assessment in der Weltgesellschaft, Berlin: Edition Sigma, 381-391.

Barben, D., Fisher, E., Selin, C., Guston, D. H., 2008, Anticipatory Governance of Nanotechnology. Foresight, Engagement, and Integration, in: Hackett, E. J., Amsterdamska, O., Lynch, M. und Wajcman, J. (Hg.): The Handbook of Science and Technology Studies, Cambridge, London: MIT Press, 979-1000.

Bora, A., Reinhardt, C., Henkel, A. (Hg.), 2012, Wissensregulierung und Regulierungswissen, Weilerswist: Velbrück.

Böschen, St., Viehöver, W., Zinn, J., 2003, Rinderwahnsinn. Können Gesellschaften aus Krisen lernen? Berliner Journal für Soziologie 13(1), 35-58. 
Bröchler, St., 2007, Technik, in: Benz, A., Lütz, S., Schimank, U. und Simonis, G. (Hg.): Handbuch Governance. Theoretische Grundlagen und empirische Anwendungsfelder, Wiesbaden: Verlag für Sozialwissenschaften, 413-423.

Eurocontrol, 2010, Communications, Navigation and Surveillance. At the heart of the future ATM system (Skyway magazine, 54).

$<$ http://www.eurocontrol.int/mil/gallery/content/public/milgallery/ documents/SW54_Low_Mag.pdf>.

Hutter, M., 1989, Die Produktion von Recht. Eine selbstreferentielle Theorie der Wirtschaft, angewandt auf den Fall des Arzneimittelpatentrechts, Tübingen: Mohr.

Mayntz, R., 1993, Policy-Netzwerke und die Logik von Verhandlungssystemen, in: Héritier, A. (Hg.): Policy-Analyse. Kritik und Neuorientierung, Opladen: Westdeutscher Verlag, 39-56.

Mayntz, R., Scharpf, F. W., 2005, Politische Steuerung - Heute? Zeitschrift für Soziologie 34(3), 236-243.

Miaillier, B., 2011, A single programme to build a Single European Sky. An interview with Bernard Miaillier, Deputy Director, Cooperative Network Design Directorate of Eurocontrol. http://www.sesarju.eu/players/experts/single-programme-build-single-european-sky--373.

Rip, A., 2006, A co-evolutionary approach to reflexive governance - and its ironies, in: Voß, J.-P., Bauknecht, D. und Kemp, R. (Hg.): Reflexive Governance for Sustainable Development. Cheltenham, Northampton: Edward Elgar, 82-100.

Schimank, U., 1992, Spezifische Interessenkonsense trotz generellem Orientierungsdissens. Ein Integrationsmechanismus polyzentrischer Gesellschaften, in: Giegel, H.-J. (Hg.): Kommunikation und Konsens in modernen Gesellschaften, Frankfurt a. M.: Suhrkamp, 236-275.

Schimank, U., 2009, Wie sich funktionale Differenzierung reproduziert: eine akteurtheoretische Erklärung, in: Hill, P., Kalter, F., Kopp, J., Kroneberg, Cl. und Schnell, R. (Hg.): Hartmut Essers Erklärende Soziologie, Frankfurt a. M., New York: Campus, 191-216.

Schimank, U., 2011, Wie Akteurkonstellationen so erscheinen, als ob gesellschaftliche Teilsysteme handeln - und warum das gesellschaftstheoretisch von zentraler Bedeutung ist, in: Albert, G. und Sigmund, St. (Hg.): Soziologische Theorie kontrovers, Wiesbaden: Verlag für Sozialwissenschaften (Kölner Zeitschrift für Soziologie und Sozialpsychologie Sonderheft, 50), 462-471.

Teubner, G., 1989, Recht als autopoietisches System, Frankfurt a. M.: Suhrkamp.

Teubner, G., 1998, Verrechtlichung - ein ultrazyklisches Geschehen: Ökologische Rekursivität im Verhältnis Recht und Gesellschaft, in: Voigt, R. (Hg.): Evolution des Rechts, Baden-Baden: Nomos, 193-213.

Teubner, G., 1999, Eigensinnige Produktionsregimes: Zur Ko-evolution von Wirtschaft und Recht in den varietiesofcapitalism. Soziale Systeme 5(1), 7-26.

Wedel, H. von, 2001, Organisation des gesundheitlichen Verbraucherschutzes (Schwerpunkt Lebensmittel), Stuttgart, Berlin, Köln: Kohlhammer.

Willke, H., 1992, Ironie des Staates. Grundlinien einer Staatstheorie polyzentrischer Gesellschaft, Frankfurt a. M.: Suhrkamp.

Willke, H. 1997, Supervision des Staates, Frankfurt a. M.: Suhrkamp. 


\title{
Leitorientierte Technologiegestaltung als Beitrag zur Umsetzung des Vorsorgeprinzips
}

\section{Urte Brand, Arnim von Gleich}

Fachgebiet Technikgestaltung und Technologieentwicklung, Universität Bremen

\section{Keywords}

Technikgestaltung, Leitbilder, Leitorientierungen, systemische Innovationen, Energiesystem

\begin{abstract}
Die TA hilft dabei, unerwünschte Neben- und Folgewirkungen technologischer Innovationen aufzudecken. Hierbei können zwei Ansätze verfolgt werden: Die Technikfolgenforschung, mit der am Anfang eines Innovationsprozesses versucht wird die Folgen einer Technologie abzuschätzen, was jedoch mit großen Unsicherheiten verbunden ist. Und die Technikbewertung, die dazu dient am Ende eines Innovationsprozesses die zu erwartenden Folgen zu bewerten, wenn jedoch die Interventionsmöglichkeiten nur noch gering sind. Um diesem Dilemma entgegen zu wirken, sollten Innovationsprozesse bereits von Anfang an so gestaltet werden, dass wichtige Aspekte wie Umwelt- und Sozialverträglichkeit im Sinne des Vorsorgeprinzips berücksichtigt werden. In diesem Beitrag werden Möglichkeiten der Technologiegestaltung bzw. der Richtungsgebung von Innovationsprozessen und insbesondere der Leitbildansatz näher beleuchtet. Wichtige Erfolgsfaktoren von Leitbildern werden mithilfe des sogenannten Dreiebenenmodells erläutert, das eine Differenzierung in Weltbilder, Leitkonzepte und Gestaltungsleitbilder vornimmt und übergeordnet von Leitorientierungen ausgeht. Schließlich wird an konkreten Praxisbeispielen im Energiebereich aufgezeigt, wie systemische Innovationsprozesse erfolgreich mithilfe von Leitorientierungen gestaltet werden können.
\end{abstract}

\section{Technology Design by Guiding Orientations as a Contribution to Implementing the Precautionary Principle}

Keywords: Technology design, guiding principles, guiding orientations, systemic innovations, energy sector

Abstract: Technology assessment can be applied to uncover undesirable side- and follow-up effects of technological innovations. There are two fundamental approaches: firstly, assessing the impacts of technologies at the beginning of innovation processes, an approach that suffers from major uncertainties. And secondly, assessing the expected impacts of technologies at the end of innovation processes, when the options for intervention are unfortunately limited. To overcome this dilemma, we suggest that innovation processes should be designed or guided at an early stage, considering important aspects such as environmental and social compatibility in accordance with the precautionary principle. This contribution discusses options and potentials for technology design by guiding principles. In addition to a discussion of functions, relevant success factors of guiding principles are described by the so-called three level model which is based on world views, guiding concepts and guiding design principles we together call guiding orientations. Finally, we demonstrate how systemic innovation processes can be designed successfully with the help of guiding orientations illustrated by practical examples from the energy sector. 


\section{Einleitung'}

Zunehmend wird eine Prävention von nicht intendierten Technologiefolgen und -risiken gefordert. Mit Technologien sind hierbei technische Entwicklungen und die zugrunde liegenden wissenschaftlichen Erkenntnisse gemeint. Jedoch stellt sich eine solche Realisierung des Vorsorgeprinzips als sehr schwierig dar. Die Technikfolgenabschätzung (TA) befindet sich sozusagen in einem Dilemma (Collingridge 1980): Am Anfang eines Innovationsprozesses sind die Einflussmöglichkeiten auf eine Technologie noch sehr hoch, jedoch ist das Wissen über die mit der Technologie verbundenen späteren Risiken und Nebenfolgen in dieser Phase sehr gering. Die im Rahmen einer solchen antizipativen Technikfolgenforschung gemachten Prognosen sind nur mit sehr großen Unsicherheiten verbunden. Dagegen sind mithilfe von Technikbewertungen wie Risikoanalysen, Ökobilanzen und toxikologische Analysen, die erst sehr spät im Innovationsprozess ansetzen, unerwünschte Folgen einer Technologie besser erkennbar. Jedoch sind die Interventionsmöglichkeiten am Ende eines Innovationsprozesses nur noch gering, da Unternehmen dann bereits sehr viel in die Entwicklung der Technologie investiert haben und Änderungen nur mit sehr hohen Kosten verbunden wären.

Somit ist es sinnvoll, bereits früh, also schon während der Forschungs- und Entwicklungsphase, den Innovationsprozess so zu gestalten, dass wichtige Aspekte wie Umwelt- oder Sozialverträglichkeit berücksichtigt werden. Durch dieses frühe Eingreifen soll die Möglichkeit eröffnet werden, nicht intendierte Folgen von vornherein zu minimieren und damit das Vorsorgeprinzip zu realisieren. Folglich rücken Fragen nach Möglichkeiten und Grenzen einer gezielten Technologiegestaltung und der damit verbundenen Beeinflussung der Innovationsrichtung immer stärker in den Vordergrund.

Die nachfolgenden Kapitel beschäftigen sich mit diesen Einflussmöglichkeiten und besonders mit der Beeinflussung durchLeitbilder. Es werden die damit verbundenen Möglichkeiten (Funktionen von Leitbildern) erläutert und Differenzierungen von Leitbildern (Dreiebenenmodell) vorgenommen, um die Voraussetzungen für erfolgreiche Leitbilder zu veranschaulichen. In diesem Zusammenhang wird davon ausgegangen, dass unterschiedliche Arten von Leitbildern existieren, nämlich Weltbilder, Leitkonzepte und Gestaltungsleitbilder, die übergeordnet als Leitorientierungen bezeichnet werden. Anschließend erfolgt die Vorstellung von Ergebnissen einer retrospektiven Analyse von erfolgreichen Leitorientierungsprozessen, um aufzuzeigen wie Leitorientierungen und insbesondere Leitkonzeptekonkret zur Gestaltung von Technologien eingesetzt werden können. Zuletzt sollen die wesentlichen Erkenntnisse dieser Arbeit dargestellt und ein Ausblick der weiteren Forschungsarbeiten gegeben werden.

1 Die dem Beitrag zugrunde liegenden Forschungsarbeiten wurden vom BMBF im Rahmen des Projektes nordwest2050 finanziert und an der Universität Bremen, Fachgebiet Technikgestaltung \& Technologieentwicklung durchgeführt. 


\section{Möglichkeiten der Gestaltung und Richtungsgebung von technologischen Innovationsprozessen}

Ansätze, die sich mit den Voraussetzungen erfolgreicher Innovationen beschäftigen, sind vielfach in der gegenwärtigen Literatur zu finden. Zahlreiche Untersuchungen befassen sich eingehend mit den Gründen für das Scheitern von Innovationen und zeigen diese detailliert auf.

Jedoch beschäftigen sich relativ wenige Ansätze mit der Gestaltung bzw. Beeinflussbarkeit von Innovationsprozessen und deren Richtungsgebung, um nicht-intendierte Folgen einer Innovation von vornherein zu minimieren und sicherzustellen, dass ein erfolgreich durchgesetztes Innovationsvorhaben am Ende auch tatsächlich zu den übergeordneten Zielsetzungen beiträgt, die mit ihm beabsichtigt waren.

Wertet man Arbeiten zur Richtungssicherheit in Innovationsprozessen im deutschsprachigen Raum aus (Behrendt 2006, Fichter 2005, Fichter et al. 2006, Gleich v. 1997, Gleich v. 1999, Klemmer 1999, Klemmer et al. 1999, Konrad und Nill 2001, Kemp und Rotmans 2005, Paech 2005a, Paech 2005b, Weber und Hemmelskamp 2005), dann lassen sich vier grundsätzliche Möglichkeiten zur Richtungsgebung von Innovationen unterscheiden:

1. Leitplanken (v. a. Regulation),

2. Anreizsysteme des Staates,

3. Leitakteure im Innovationsprozess und

4. Leitbilder.

Bei diesen Ansätzen gehen wir davon aus, dass sie nicht unabhängig voneinander zum Tragen kommen, sondern gemeinsam ihren Einsatz finden.

$\mathrm{Zu}$ den ersten drei aufgeführten Möglichkeiten der Richtungsgebung ist schon häufiger publiziert worden (u. a. Cooper 2001, Fichter 2005, Hauschildt und Salomo 2010, Klemmer et al. 1998, Schwarz und Linne 2003), so dass hier das Hauptaugenmerk auf die Möglichkeiten und Grenzen der Richtungsgebung mit Hilfe von Leitbildern gerichtet werden soll. Daher sollen nachfolgend nur kurz die drei erstgenannten Ansätze erläutert werden und anschließend eine intensivere Auseinandersetzung mit dem Leitbildansatz, der sogenannten leitbildorientierten Technologiegestaltung, erfolgen.

\section{I Leitplanken}

Das Instrument der Leitplanken trägt zur Risikovorsorge bei Leitplanken haben insbesondere die Aufgabe den Suchraum bezogen auf mögliche Innovationen abzusichern und gegenüber nicht tolerierbaren Gefährdungen zu begrenzen. Sie bewahren davor, dass die Tragekapazitäten von ökonomischen, sozialen und ökologischen Subsystemen überschritten werden (Gleich v. et al. 2010a). Somit können sie verhindern, dass sehr risikobehaftete Technologien mit schwer abschätzbaren und sehr weit reichenden Folgen wie z. B. Technologien mit einer großen Eingriffstiefe (z. B. Geo-Engineering) entwickelt werden, bei deren Einsatz im Falle unerwünschter Folgen kaum noch korrigierend eingegriffen werden kann.

Gesetzt werden Leitplanken von Instanzen und Organisationen, die nicht selbst innovieren, aber in Interaktion mit den Personen und Organisationen im Innovationsprozess stehen. 
Unter ihnen sind insbesondere staatliche Regulierungen in Form von Richtlinien, Verordnungen oder Gesetzen zu verstehen wie z. B. „Regulierungen von Stoffeinträgen in die Umwelt“ oder „Regulierungen des Anbaus von Bioenergiepflanzen“.

\subsection{Staatliche Anreizsysteme}

Ein weiteres Instrument, um Innovationsprozessen eine Richtung geben zu können, stellen die Anreizsysteme des Staates dar. Mit Hilfe von Anreizen in Form von Forschungs- und Förderprogrammen, Subventionen, Steuervergünstigungen etc. ist es dem Staat möglich, bestimmte Innovationen verstärkt zu fördern. Dies können bspw. das vom Bundesministerium für Umwelt, Naturschutz und Reaktorsicherheit geförderte Projekt „100 \% Erneuerbare-Energie-Regionen“ oder die „Steuerliche Förderung von energetischen Sanierungsmaßnahmen an Wohngebäuden“ sein.

\subsection{Leitakteure}

Im Innovationsprozess können auch einzelne Akteure eine wichtige richtungsweisende Funktion übernehmen. Diese Akteure werden oft als sogenannte Promotoren oder Schlüsselakteure bezeichnet, die den Innovationsprozess aktiv mitgestalten und seine Durchführung durch die Überwindung von Widerständen und Barrieren unterstützen. Dabei wirken sie durch ihre Überzeugungskraft und ihre soziale Ausstrahlung als Meinungsführer auf andere Beteiligte im Innovationsprozess (Fichter et al. 2010b).

\subsection{Leitbilder}

Neben den bereits genannten Möglichkeiten zur Richtungsgebung von Innovationsprozessen können auch die sogenannten Leitbilder eine Richtung für den Innovationsprozess vorgeben. Unter Leitbildern sind gemeinsame Zielvorstellungen zu verstehen, die gleichzeitig erwünscht und für machbar gehalten werden. Sie prägen die Wahrnehmung sowie das Denken, Entscheiden und Handeln von individuellen und kollektiven Akteuren (Giesel 2007). Bekannte Leitbilder sind bspw. „Nachhaltigkeit“" oder ,Grüne Chemie“.

Bereits in den 1990er Jahren gab es eine vergleichsweise intensive Forschungsaktivität zur Wirkung von Leitbildern in der Technikentwicklung im Rahmen der sogenannten Technikgeneseforschung (vgl. Dierkes 1992, 1997). So führten Technikforscher zahlreiche retrospektive Untersuchungen z. B. in der Informatik und Computertechnik durch (vgl. Hellige 1996, Mambrey et al. 1995, Pflüger 1995, Rolf et al. 1990), aber auch in der Konstruktionsmethodik (vgl. Dierkes 1994, Hellige 1991) und im Fahrzeugbau (vgl. Canzler und Knie 1994, Koolmann 1992), um die Rolle von Leitbildern in technischen Entwicklungsprozessen zu analysieren.

Jedoch blieb weitestgehend ungeklärt, ob eine gezielte Gestaltung von Technologien mit Hilfe von Leitbildern von Anfang an möglich sei. Zwar wurde darauf hingewiesen, dass die Möglichkeit zur Technik- bzw. Technologiegestaltung mit Hilfe von Leitbildern keinesfalls überschätzt werden dürfe (u. a. Hellige 1996), und einige retrospektive Analysen sprachen dafür, dass sich Leitbilder erst im Laufe des technischen Entwicklungsprozesses herausbilden würden (Maaß 1994, Tepper 1994), doch gibt es hier noch weiteren Forschungsbedarf: Damals wurden besonders kleinschrittige technologische Innovationen ex-post untersucht. Systemische Innovationen, die mehrere Innovationsarten - technologische, organisationale, institutionelle und soziale Innovationen - bündeln 
(Karlstetter et al. 2010), waren hingegen nicht Gegenstand der Untersuchungen. Heutzutage ist außerdem eine zunehmende Beteiligung von gesellschaftlichen und politischen Akteuren an Innovationsprozessen zu beobachten. Nicht mehr nur die technischen Eliten haben Interesse an technologischen Entwicklungen, sondern verstärkt auch die Öffentlichkeit. Man denke bspw. an die dezentrale Energieversorgung, an der nicht mehr nur die Energieversorger beteiligt sind, sondern auch verschiedenste Stakeholder wie private Haushalte, Handwerkskammern, Nichtregierungsorganisationen usw. Somit lohnt es sich, die Möglichkeiten und Grenzen der Gestaltung von systemischen Innovationsprozessen mit Hilfe von Leitbildern genauer auszuloten.Doch zunächst soll der Fokus auf die Funktionen von Leitbildern gerichtet werden, um ein besseres Verständnis dafür zu bekommen, wie Leitbilder in Innovationsprozessen wirken können.

\subsection{Funktionen von Leitbildern}

In der Literatur sind viele Studien zu finden, die sich intensiv mit den Funktionen von Leitbildern auseinandergesetzt haben (u. a. Ahrens und Gleich v. 2002, Dierkes et al. 1992, Giesel 2007, Knieling 2006). So werden Leitbildern insbesondere folgende sechs Funktionen zugeschrieben:

1. Koordination und Synchronisation von Akteursaktivitäten: Leitbilder unterstützen Akteure bei der Fokussierung auf ein gemeinsames Ziel. Damit können sie neues technisches Wissen generieren und die Koordination und Synchronisation der Akteursaktivitätenvereinfachen.

2. Steigerung der Motivation: Leitbilder sprechen Akteure emotional an, wecken dadurch ihr Engagement und steigern somit die Motivation der Akteure.

3. Schaffung einer Gruppenidentität: Leitbilder stellen einen kulturellen Kontext her. Werden sie als gemeinsame Leitbilder wahrgenommen, kann eine Gruppenidentität geschaffen bzw. diese erhöht werden.

4. Verringerung der wahrgenommenen Komplexität: Leitbilder können die wahrgenommene Komplexität von Innovationsprozessen reduzieren, indem sie denk- und handlungsleitend sind. Sie geben einen Weg für die Akteure vor.

5. Strukturierung der Wahrnehmung: Leitbilder geben dem Handeln einen Sinn und strukturieren damit die Wahrnehmung sowie das Denken und Handeln der Akteure. Im günstigen Fall werden sie ,gelebt“".

6. Schaffung von Orientierung: Leitbilder unterstützen die interne Abstimmung von Zielen. Sie geben eine gemeinsame und übergreifende Orientierung und können somit Unsicherheiten und Wissenslücken überbrücken.

Auf Grundlage dieser Erkenntnisse können Leitbilder folgendermaßen definiert werden:

,[...]leitbilder beeinflussen als Ausdruck einer wünschenswerten und gleichzeitig machbaren Entwicklung die Wahrnehmung sowie das Entscheiden und Handeln von Individuen und Gruppen. Sie können auf diese Weise Innovationsprozesse vorantreiben und ausrichten, indem sie helfen Orientierung zu geben, die Komplexität zu reduzieren und Unklarheiten mit Blick auf mögliche Innovationsfolgen zu überbrücken“" (Gleich v. et al. 2010b:139).

\subsubsection{Differenzierung von Leitbildern - Dreiebenenmodell}

Es fällt auf, dass in der Literatur oftmals Differenzierungen von Leitbildern vorgenommen werden. So spricht Zieschank bspw. nicht mehr nur von Leitbildern, sondern zusätzlich von Leitlinien und Leitzielen, die sich alle drei durch ihren Konkretisierungsgrad unterscheiden lassen (Zieschank 1999). Ein bekanntes Beispiel hierfür ist das Leitbild „Nachhaltigkeit“, das sich durch untergeordnete Leitbilder wie „,regenerierbare Ressourcen“ oder „Sozialverträglichkeit“ konkretisieren lässt. 
Mit Ausnahme der Überlegungen zur Nachhaltigkeit bleibt jedoch in der Literatur weitestgehend ungeklärt, welche Zusammenhänge zwischen über- und untergeordneten Leitbildern bestehen, wie sich Konkretisierungen in Form von untergeordneten Leitbildern entwickeln und wie die verschiedenen Typen allgemein unterschieden werden können. Diesen Fragen haben wir uns angenommen und ein Dreiebenenmodell ${ }^{2}$ entwickelt, das eine Differenzierungsmöglichkeit für verschiedene Leitbilder bietet (siehe Abbildung 1).

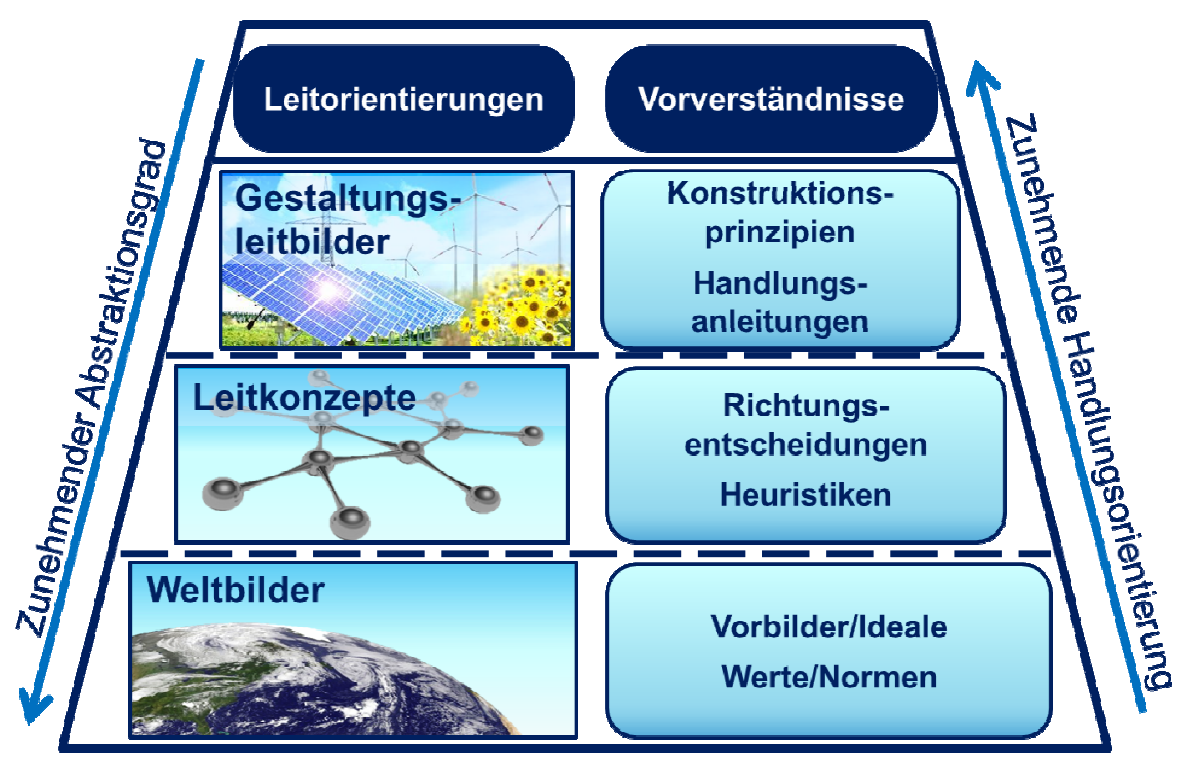

Abbildung 1: Dreiebenenmodell (eigene Darstellung)

Eine Besonderheit dieses Modells ist die Tatsache, dass wir nun nicht mehr von Leitbildern sprechen, sondern von übergeordneten Leitorientierungen, die sich nach drei Ausprägungsebenen unterscheiden lassen:

1. Auf der untersten Ebene sind die Weltbilder ${ }^{3}$ angesiedelt. Sie sind oft holzschnittartig und besitzen einen hohen Grad an Abstraktion. Unter Weltbildern sind Werte, Normen, Vorbilder und Ideale zu verstehen, wie z. B. Vorstellungen von einem ,richtigen Umgang mit der Natur“ oder von einer, ,guten Technik“.

2. Auf der mittleren Ebene befinden sich die Leitkonzepte, die schon weniger abstrakt sind, mit denen jedoch noch keine detaillierten Vorstellungen von Umsetzungsmöglichkeiten verbunden sind. Sie geben eher eine Richtung bzw. ein Ziel vor und können somit als Richtungsentscheidungen oder Heuristiken bezeichnet werden. Zu den Leitkonzepten zählen bspw. „Nachhaltigkeit“, „Kreislaufwirtschaft“ oder „Solares Wirtschaften“.

3. Auf der dritten Ebene sind schließlich die Gestaltungsleitbilder angesiedelt, die die Leitkonzepte konkretisieren und somit einen hohen Grad an Handlungsorientierung besitzen. Sie fokussieren auf Konstruktionsprinzipien oder Handlungsanleitungen, mit denen z. B. eine „Ökologische Landwirtschaft“ oder „Smart Systems“ konkret umgesetzt werden können.

2 Siehe dazu Gleich v. et al. (2010b), Beucker et al. (2011).

3 Joseph Huber hat sich in seinem Buch „Technikbilder - Weltanschauliche Weichenstellungen der Technologie- und Umweltpolitik' insbesondere mit dieser grundlegenden Ebene beschäftigt (Huber 1989); siehe ebenso Hofkirchner (1996). 
Wir gehen davon aus, dass Gestaltungsleitbilder und Leitkonzepte hinsichtlich ihres wertbezogenen und emotionalen Inhalts und damit auch hinsichtlich ihrer Wirkung erst mit Bezug auf die darunter liegenden Weltbilder verstanden werden können. Viele besonders wirksame Gestaltungsleitbilder und Leitkonzepte beziehen ihre emotionale Aufladung, ihren gesellschaftlichen Resonanzboden und ihre Ausprägung bzw. Gestalt ${ }^{4}$ von solchen Weltbildern. In diesem Zusammenhang nehmen wir an, dass gesellschaftliche Resonanz oftmals auf tiefverwurzelten Weltbildern beruht, die durch kollektive Erfahrungen geprägt sind und mit denen Vorstellungen von einem ,richtigen Umgang mit Natur“, von einer „guten Technik“ oder gar von einem „guten Leben“ transportiert werden. Leitorientierungen knüpfen somit an im kollektiven Gedächtnis tiefverankerte Wünsche wie dem „Traum vom Fliegen“ oder „einem Leben in Einklang mit der Natur“. Damit besitzen sie einen starken emotionalen Bezug (Gleich v. et al. 2010b).

Unterscheiden lassen sich die Leitorientierungen auf den verschiedenen Ebenen durch ihren unterschiedlichen Grad an Abstraktion bzw. Ausdifferenzierung. Weltbilder sind oft holzschnittartig, während Gestaltungsleitbilder stark ausdifferenziert sein können. Außerdem lassen sich die verschiedenen Typen von Leitorientierungen aufgrund ihrer unterschiedlichen gesellschaftlichen, räumlichen und zeitlichen Reichweite voneinander abgrenzen. Weltbilder können gesellschaftlich und räumlich sehr weit verbreitet sein. Sie wirken langfristig und können auch nicht ohne weiteres beeinflusst bzw. verändert werden. Dagegen haben Leitkonzepte eher eine mittlere und Gestaltungsleitbilder eine vergleichsweise kurze gesellschaftliche, räumliche und zeitliche Reichweite (Gleich v. et al. 2010b).

Zum besseren Verständnis des Dreiebenenmodells soll nachfolgend ein Beispiel für die mögliche Einordnung einer ausgewählten Leitorientierung in das Modell gegeben werden. Als Fallbeispiel soll die Leitorientierung „Energieautarkie“ dienen, die in den letzten Jahren wieder zunehmend an Bedeutung gewonnen hat. Mittlerweile gibt es zahlreiche Regionen in und außerhalb von Deutschland, die sich eine autarke Energieversorgung zum Ziel gesetzt haben.

Wir sind der Ansicht, dass Energieautarkie den Leitkonzepten zugeordnet werden kann, da die Leitorientierung bereits eine Richtung vorgibt, in der sich die Energieversorgung entwickeln soll. Mit dem Streben nach Energieautarkie wird der Wunsch geäußert, unabhängig von externen Energieversorgern zu sein. Jedoch stellt die Leitorientierung noch keine Konkretisierung dar. Bspw. bleibt offen, wie die angestrebte Energieautarkie umgesetzt werden kann, z. B. ob fossile oder erneuerbare Energieressourcen verwendet werden.

Interessant erscheint in diesem Zusammenhang besonders, welche Weltbilder dann hinter dem Leitkonzept stehen. Energieautarkie ist stark mit dem Wunsch nach Unabhängigkeit verbunden, wobei zumindest im deutschsprachigen Raum neben der positiv interpretierten Vorstellung von „Autonomie" auch immer eine eher problematische kriegswirtschaftliche Bedeutung mitschwingt. In der Entwicklungspsychologie wird davon ausgegangen, dass Menschen im Verlauf ihrer Entwicklung vom Kindes- bis zum Erwachsenenalter mehr und mehr nach Unabhängigkeit streben (vgl. Berk et al. 2011). Positiv wieder aufgegriffen wurden diese Aspekte insbesondere in den 1970er und 1980er Jahren im Sinne der „self reliance“ und der eigenständigen Regionalentwicklung. Über die letztgenannten gelang es auch gegenläufige Bestrebungen zu integrieren, z. B. das Bedürfnis nach Vernetzung.

Was die Ebene der Gestaltungsleitbilder betrifft, können selbstverständlich sehr unterschiedliche Handlungsanleitungen und Konstruktionsprinzipien aus dem Leitkonzept Energieautarkie abgeleitet werden. Bspw. ist es möglich, Energieautarkie näher zu konkretisieren, indem auf die vor Ort

4 Gestalt ist hier im Sinne der Gestaltpsychologie zu verstehen. Der Gestaltbegriff ist wesentlich geeigneter für den Ausdruck des hier „Gemeinten“ als der Begriff des „Bildes“, wie er im Begriff „Leitbild“ transportiert wird. 
verfügbaren Energieressourcen zurückgegriffen wird, mit denen das Leitkonzept realisiert werden soll. Dies können bspw. erneuerbare Ressourcen sein und ein abgeleitetes Gestaltungsleitbild könnte dann „100 \% Erneuerbare Energien“ heißen. 100 \% Erneuerbare Energien ist ein Gestaltungsleitbild, das bereits eine relativ hohe Handlungsorientierung aufweist, jedoch bleibt noch offen, mit welchen erneuerbaren Energiequellen, ob z. B. mit nachwachenden Rohstoffen oder mit Windkraft, das Leitkonzept Energieautarkie umgesetzt wird.

\section{Retrospektive Analyse von erfolgreichen Leitkonzepten in systemischen Innovationsprozessen}

Um eine Antwort auf die Eingangsfrage zu finden, inwiefern es möglich ist, systemische Innovationsprozesse mit Hilfe von Leitbildern bzw. Leitorientierungen zu gestalten und ihnen eine Richtung zu geben, haben wir uns mit dem Entstehungsprozess von Leitkonzepten und deren Wirkung und Umsetzung in systemischen Innovationsprozessen auseinandergesetzt. Dafür haben wir eine retrospektive Analyse von erfolgreichen Leitkonzepten durchgeführt, die zur Gestaltung von systemischen Innovationsprozessen ihren Einsatz fanden.

In den weiteren Abschnitten möchten wir diese Analyse näher erläutern und uns dabei besonders auf das bereits vorgestellte Leitkonzept „Energieautarkie“ beziehen. In diesem Zusammenhang gehen wir davon aus, dass Innovationsprozesse, die auf Energieautarkie zielen, als systemische Innovationsprozesse bezeichnet werden können, da es bei ihnen nicht nur um technologische Änderungen (u. a. Photovoltaik/Windkraftanlagen), sondern auch um akteursstrukturelle bzw. soziale (u. a. finanzielle/symbolische Förderung durch Öffentlichkeit, Konsument wird zum Prosumer) und institutionelle Änderungen (u. a. staatliche Förderung) geht (vgl. Konrad und Scheer 2003, Fichter und Hintemann 2009).

Nachfolgend soll zunächst das zugrundeliegende Forschungsdesign (Untersuchungsgegenstand, Forschungsfragen, Datenbasis) der Analyseerläutert werden. Danach folgendie Analyseergebnisse, welche die Entstehung und Umsetzung des Leitkonzepts in Anlehnung an ein Phasenmodell darstellen.

\section{I Forschungsdesign}

Zur Analyse des Leitkonzepts wurden drei Regionen untersucht, die Energieautarkie bereits erfolgreich umgesetzt haben. Bei den untersuchten Regionen handelt es sich um den Stadtteil Dardesheim in Sachsen-Anhalt, die Stadt Güssing in Österreich und die dänische Insel Samsø. Bei der Analyse standen insbesondere drei Kernfragen im Fokus der Betrachtung:

1. Wie fand das Leitkonzept Eingang in die Regionen?

2. Auf welche Weise konnte das Leitkonzept verbreitet werden?

3. Welche Akteure waren maßgeblich an der Entstehung, Verbreitung und Umsetzung des Leitkonzepts beteiligt?

Zur Beantwortung dieser Fragen wurden wissenschaftliche Publikationen und Forschungsberichte sowie Zeitungsartikel und schriftliche Interviews herangezogen (siehe 3.2). 


\subsection{Ergebnisdarstellung anhand eines Phasenmodells}

Die Ergebnisse der Untersuchung zeigen, dass die Entwicklung des Leitkonzepts Energieautarkie in allen drei Regionen ähnlich verlief. Aus diesen Erkenntnissen heraus wurde versucht, die Entwicklung des Leitkonzepts in Phasen einzuteilen und in Anlehnung an das Stufenmodell von Dierkes et al. (1992), welches den idealtypischen Verlauf einer Leitbildkarriere darstellt, ein Phasenmodell ${ }^{5}$ entwickelt (siehe Abbildung 2).

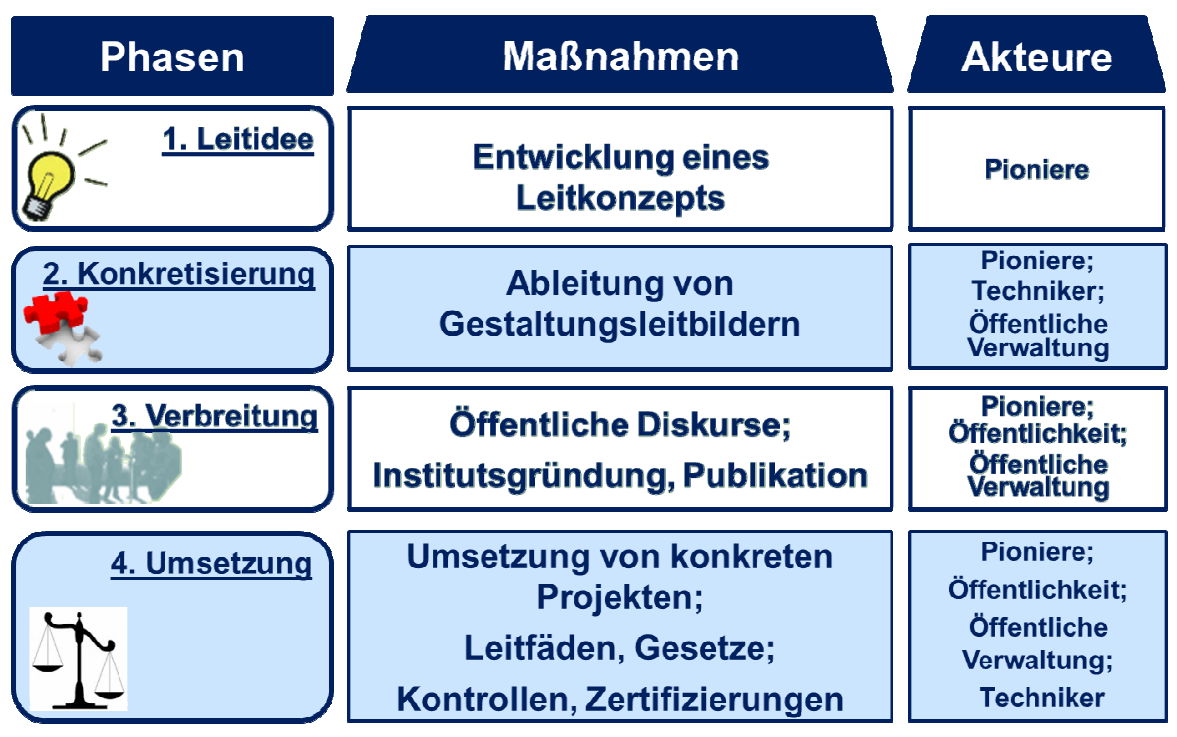

Abbildung 2: Phasenmodell: Darstellung der Entwicklung und Umsetzung eines Leitkonzepts (eigene Darstellung in Anlehnung an Dierkes et al. 1992)

\section{Phase-Leitidee:}

Charakteristisch für die erste Phase ist ein bestehendes Problem oder eine Krise.

Im Falle der untersuchten Regionen bestand dieses Problem in einer schwachen Wirtschaft verbunden mit einer hoher Arbeitslosenrate und Migration (vgl. Beermann 2009). Außerdem hatten die Regionen mit hohen Preisen für fossile Energieträger zu kämpfen.

So musste im Falle von Samsø das notwendige Heizöl mit Schiffen geliefert werden, was die Rohstoffkosten stark ansteigen ließ (vgl. Beermann 2009, Jakobsen 2008). Güssing sah die Probleme darin, vom Erdgasförderunternehmen Gazprom abhängig zu sein und damit keinen Einfluss auf die Preisgestaltung zu haben (vgl. EEE 2011).

Kennzeichnend bei allen drei untersuchten Regionen ist, dass in dieser Phase das existierende Leitkonzept, die zentrale Verbundversorgung, die bestehenden Probleme nicht mehr lösen konnte. ${ }^{6}$ Es mussten alternative Konzepte bzw. neue Ideen gefunden werden. Einzelne Personen, man kann sie Pioniere nennen, die in der Regel technisches Know-how hatten und sich selbst als Visionäre be-

5 Siehe dazu auch Gleich v. et al. (2010a); Beucker et al. (2011).

6 Baedeker (2002) hat sich in seiner Dissertation ,Leitbild und Netz - Techniksoziologische Überlegungen zur Entwicklung des Stromverbundsystems“ ausführlich mit dem Wandel des Leitbildes ,zentrale Verbundversorgung“ zum Leitbild „dezentrale Solarenergienutzung“ beschäftigt. 
zeichneten, machten sich Gedanken über die Probleme in ihrer Region (vgl. Beermann 2009, Franken 2007, Stuiber 2007). Ihrer Meinung nach konnte die Wirtschaft nur angekurbelt werden, wenn eigene, regionsspezifische Ressourcen genutzt werden und man sich unabhängig von den externen Energieversorgern macht. Das Geld sollte in der Region bleiben (vgl. Beermann 2009).

Mit dem Wunsch nach Unabhängigkeit und der Verstärkung der regionalen Wertschöpfung entstand damit das Leitkonzept Energieautarkie, das unserer Meinung nach besonders aufgrund der Verknüpfung zum Weltbild „Autonomie“ (häufig versinnbildlicht in einem kleinen gallischen Dorf) seine emotionale Aufladung beziehen und seine Wirkung entfalten konnte.

Charakteristisch für die erste Phase ist also auch die Formulierung einer existierenden und zuvor nicht ausgesprochenen Leitidee in Form eines Leitkonzepts.

\section{Phase-Konkretisierung:}

In der zweiten Phase nimmt das Leitkonzept mehr und mehr Gestalt an, und konkrete Gestaltungsleitbilder werden abgeleitet.

In den untersuchten Regionen erfolgte die Umsetzung der Energieautarkie mit Hilfe von erneuerbaren Energieressourcen, die zu $100 \%$ genutzt werden sollten. Warum die Pioniere genau zu diesem Gestaltungsleitbild kamen, hatte sehr unterschiedliche Gründe.

Im Falle von Samsø scheint ein Wettbewerb, der von der dänischen Regierung 1997 ausgeschrieben wurde, ausschlaggebend gewesen zu sein (vgl. Jakobsen 2008). Es sollte eine dänische Vorzeigeregion gefunden werden, die es schafft, in 10 Jahren zu $100 \%$ erneuerbare Energien zu nutzen. In Dardesheim sorgte dagegen die Einführung des Erneuerbaren-Energien-Gesetzes für die Umsetzung des Gestaltungsleitbildes 100 \% Erneuerbare Energien (vgl. Hoffmann 2010). Im Falle von Güssing erschien es den Pionieren besonders wichtig die Ressource zu nutzen, die in der Region in großen Mengen vorhanden ist, in diesem Falle der nachwachsende Rohstoff Holz (vgl. Stuiber 2007). Somit wurde das Leitkonzept Energieautarkie in allen Regionen mit Hilfe von erneuerbaren Energien realisiert, und das Gestaltungsleitbild „100 \% Erneuerbare Energien” setzte sich durch.

\section{Phase-Verbreitung:}

In der dritten Phase findet die Verbreitung der Gestaltungsleitbilder und der zugrundeliegenden Leitkonzepte mit Hilfe von öffentlichen und kritischen Diskursen statt.

Im Falle der untersuchten Energieregionen standen viele Bewohner dem neuen Leitkonzept und insbesondere den Gestaltungsleitbildern sehr kritisch gegenüber. Erst mit Hilfe von Informationsveranstaltungen und öffentlichen Diskursen konnten sich die Bewohner mit den neuen Umsetzungskonzepten anfreunden und sich auf ein gemeinsames Ziel verständigen (vgl. Beermann 2009, Mano 2010).

Außerdem können wissenschaftliche Publikationen unddie Gründung von Instituten mit entsprechender Orientierung die Verbreitung der Leitkonzepte und Gestaltungsleitbilder unterstützen.

So wurde im Falle von Dardesheim bspw. das Windblatt ${ }^{7}$ publiziert, das die Öffentlichkeit in regelmäßigen Abständen über den Projektstand wie die Errichtung neuer Windkraftanlagen informierte. Samsø gründete die Energieakademi ${ }^{8}$, die sich mit der Umsetzung von neuen Energieprojekten beschäftigt und als Informationsstelle für die Öffentlichkeit dient.

7 Siehe http://www.energiepark-druiberg.de/windblatt.php.

8 Siehe http://www.energiakademiet.dk/. 


\section{Phase - Umsetzung:}

In der vierten und letzten Phase werden die Gestaltungsleitbilder in konkrete Maßnahmen umgesetzt. Im Falle der Energieregionen wurden bspw. die geplanten Windparks, Photovoltaikanlagen und Fernwärmenetze errichtet (vgl. Härtel 2008, Jakobsen 2008, Koch 2006).

Auch die Erstellung von Leitfäden und die Einführung von Gesetzen können zur Umsetzung der Leitkonzepte und Gestaltungsleitbilder beitragen. Bspw. wäre es denkbar, Gesetze zu erlassen, welche die ausschließliche Nutzung des Fernwärmenetzes reglementieren oder festlegen, regenerative Energiesysteme (z. B. Photovoltaikanlagen) anstatt konventioneller Energieerzeugungsanlagen (z. B. ölbetriebene Durchlauferhitzer) zu nutzen. Letztendlich können auch vorgeschriebene Kontrollen und Zertifizierungen zur Investition in und zur Nutzung von erneuerbaren Energien beitragen.

\section{Schlussfolgerungen und Ausblick}

Die Ergebnisse der retrospektiven Analyse bestätigen die anfängliche These, dass in der Regel alle Instrumente der Richtungsgebung gemeinsamin einem Innovationsprozess zum Einsatz kommen. Zur Veranschaulichung soll dies an der Beispielregion Dardesheim gezeigt werden.

In Dardesheim konnte das Erneuerbare-Energien-Gesetz als Leitplanke und Anreiz wirken. Gleichzeitig nahmen die Pioniere als Leitakteure eine wichtige Rolle ein und gaben dem Innovationsprozess eine Richtung vor. Das Leitkonzept Energieautarkie und deren Gestaltungsleitbild 100 \% Erneuerbare Energien trug schließlich als gemeinsam wahrgenommenes Ziel zur Orientierung der Akteure bei. Mittlerweile wird das Projekt durch das Bundesministerium für Umwelt, Naturschutz und Reaktorsicherheit (BMU) gefördert ${ }^{9}$ und soll regional erweitert werden (siehe Abbildung 3).

Abschließend soll betont werden, dass mit der retrospektiven Analyse weiterer Forschungsbedarf verbunden ist. So sollen in einem nächsten Schritt die Werte und Ideale der Pioniere in persönlichen Interviews erfragt werden, um Rückschlüsse auf die dahinter stehenden Weltbilder ziehen zu können. Insbesondere sollen die Motive, welche die Pioniere zur Unterstützung der Innovationsprozesse bewegt haben, ermittelt werden.

Außerdem sollen weitere Energieregionen untersucht werden, um feststellen zu können, ob sich die Entwicklung des Leitkonzepts auf ähnliche Weise wie in den genannten Fallbeispielen vollzogen hat.

Der nächste Schritt soll dann die Analyse weiterer systemischer Innovationsprozesse sein, in denen andere Leitorientierungen eine Rolle gespielt haben bzw. spielen (z. B. „Kreislaufwirtschaft“ oder „Bionik").

Und schließlich wird auch der Versuch gewagt, prozessbegleitend die Entwicklung und Wirkung des Leitkonzepts „Resilienz“ zu untersuchen, das im Rahmen des Projektes nordwest205010 als Ziel für Anpassungsmaßnahmen an den Klimawandel in der Metropolregion Bremen-Oldenburg umgesetzt werden soll.

9 Siehe http://www.regmodharz.de/home/.

${ }^{10}$ Siehe http://www.nordwest2050.de. 


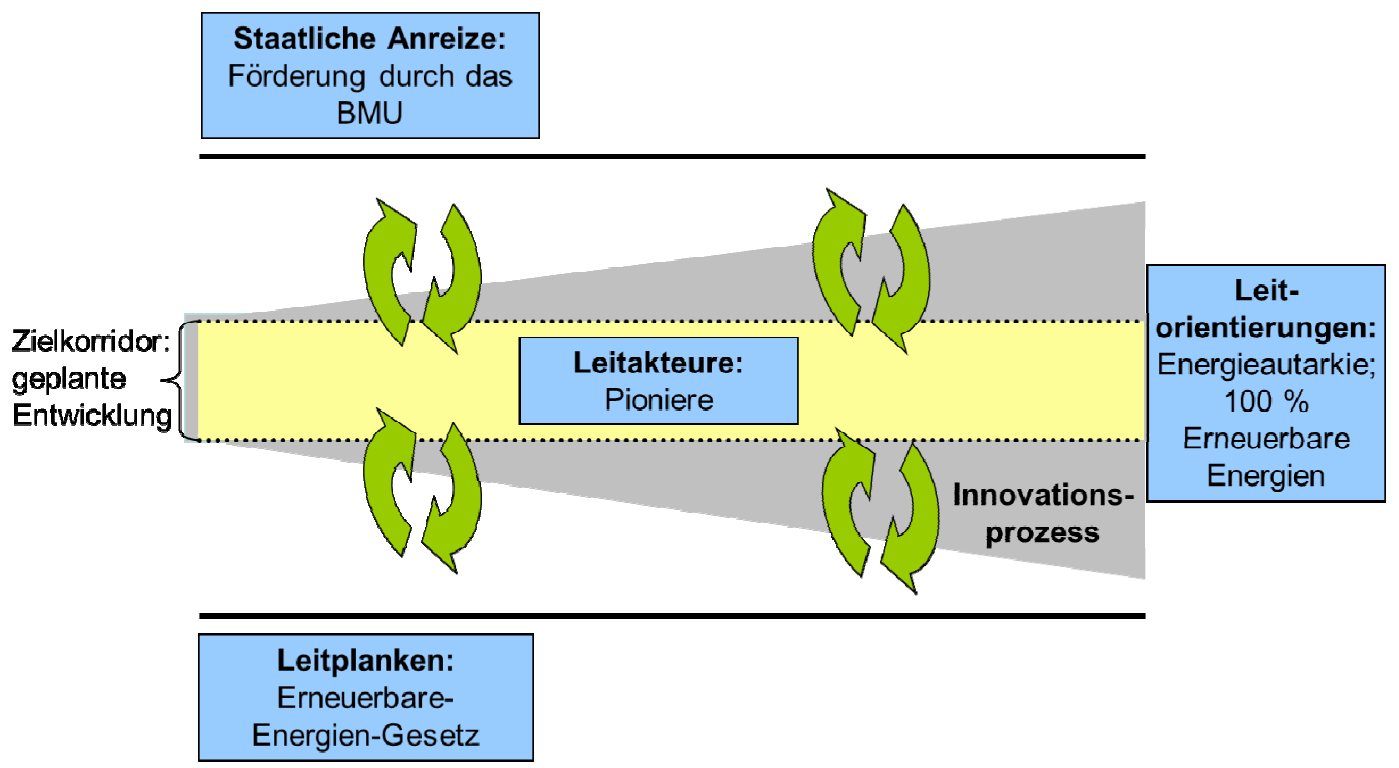

Abbildung 3: Fallbeispiel Dardesheim: Möglichkeiten der Richtungsgebung (in Anlehnung an Fichter et al. 2010a)

\section{Literatur}

Ahrens, A., Gleich, A. von, 2002, Leitbilder in der Chemikalienentwicklung und Stoffpolitik. Bericht vom SubChem-Workshop. Hamburg, verfügbar unter: http://www.tecdesign.uni-bremen.de/subchem/pdf/Leitbild_Bericht_final_0803.pdf (letzter Abruf Januar 2012).

Baedeker, H., 2002, Leitbild und Netzwerk - Techniksoziologische Überlegungen zur Entwicklung des Stromverbundsystems, Dissertation, Friedrich Alexander Universität Erlangen.

Beermann, J., 2009, 100\% Renewable Energy Regions in Europe - A comparative analysis of local renewable energy development, Freie Universität Berlin, verfügbar unter: http://seacourse.dk/download/Beermann09.pdf (letzter Abruf Oktober 2011).

Behrendt, S., 2006, Integrated Roadmapping. Unterstützung nachhaltigkeitsorientierter Innovationsprozesse in der Informationstechnik und Telekommunikation., in: Pfriem, R. et al. (Hg.): Innovationen für eine nachhaltige Entwicklung, Wiesbaden: Deutscher UniversitätsVerlag, 395-416.

Berk, L. E., Petersen, K., Aralikalti, E., Schönpflug, U., 2011, Entwicklungspsychologie. 5. akt. Aufl., München: Pearson Studium.

Beucker, S., Brand, U., Fichter, K., Gleich, A. von, 2011, Leitorientiertes integriertes Roadmapping - Konzeptionelle Grundlagen und Methode für die Entwicklung von Klimaanpassungsinnovationen, Bremen, Oldenburg: nordwest2050-Werkstattbericht 10/2011.

Canzler, W., Knie, A., 1994, Von der Automobilität zur Multimobilität. Die Krise des Automobils als Chance für eine neue Verkehrs- und Produktpolitik, in: Fricke, W. (Hg.): Jahrbuch Arbeit und Technik. Bonn: Verlag J. H. W. Dietz Nachfolger, 171-182. 
Collingridge, D., 1980, The Social Control of Technology, New York: St. Martin's Press.

Cooper, R. G., 2001, Winning at New Products: Accelerating the Process from Idea to Launch, $3^{\text {rd }}$ Edition, New York: Perseus Publishing.

Dierkes, M., 1992, Ist Technikentwicklung steuerbar? in: Bergstermann, J., Manz, T. (Hg.): Technik gestalten, Risiken beherrschen. Befunde der Sozialforschung zur Entwicklung moderner Produktionstechnik, Berlin: edition sigma, 15-35.

Dierkes, M., 1994, Leitbilder, Organisationskultur und Organisationshandeln, in: Pahl, G. (Hg.): Psychologische und pädagogische Fragen beim methodischen Konstruieren: Ergebnisse des Ladenburger Diskurses vom Mai 1992 bis Oktober 1993, Köln: Verlag TÜV Rheinland, 198-213.

Dierkes, M., 1997, Technikgenese. Befunde aus einem Forschungsprogramm, Berlin: edition sigma.

Dierkes, M., Hoffmann, U., Marz, L., 1992, Leitbild und Technik. Zur Genese und Steuerung technischer Innovationen, Berlin: edition sigma.

EEE - Europäisches Zentrum für Erneuerbare Energie GmbH, 2011, Entwicklung der Erneuerbaren Energie in Güssing, verfügbar unter: http://www.guessing.co.at/downloads/ Infotxt_ErneuerbareEnergie.pdf (letzter Abruf Januar 2012).

Fichter, K., 2005, Interpreneurship. Nachhaltigkeitsinnovationen in interaktiven Perspektiven eines vernetzenden Unternehmertums, Habilitationsschrift, Marburg: Metropolis-Verlag,

Fichter, K., Hintemann, R., 2009, Innovationsmanagement - Kapitel 1: Innovation: Was sie ist und warum sie zählt. Vorlesungsunterlagen. Carl von Ossietzky Universität Oldenburg, verfügbar unter: http://www.innovationsmanagement.uni-oldenburg.de/download/ leseproben/p0I_KapI.pdf (letzter Abruf Januar 2012).

Fichter, K., Hintemann, R., Stecher, T., 2010a, Richtungsgebung als Kontextsteuerung: ein integratives Modell, in: Fichter, K., Gleich, A. von, Pfriem, R., Siebenhüner, B. (Hg.): Theoretische Grundlagen für erfolgreiche Klimaanpassungsstrategien, Bremen, Oldenburg: nordwest2050-Bericht 1/2010, 163-164.

Fichter, K., Hintemann, R., Stecher, T., 2010b, Leitakteure: Richtungsgebende Schlüsselakteure, in: Fichter, K., Gleich, A. von, Pfriem, R., Siebenhüner, B., (Hg.): Theoretische Grundlagen für erfolgreiche Klimaanpassungsstrategien,Bremen, Oldenburg: nordwest2050-Bericht 1/2010, 154-162.

Fichter, K., Noack, T., Beucker, S., Bierter, W., Springer, S., 2006, Nachhaltigkeitskonzepte für Innovationsprozesse, Stuttgart: Fraunhofer IRB Verlag.

Franken, M., 2007, Wir haben hier ein Kleinod geschaffen, verfügbar unter: http://www.vdi-nachrichten.com/artikel/Wir-haben-hier-ein-Kleinod-geschaffen/32789/I, zuletzt aktualisiert am 29.10.2011 (letzter Abruf Januar 2012).

Gleich, A. von, 1997, Innovationsfähigkeit und Richtungssicherheit. Voraussetzungen für die ökologische Modernisierung der bundesrepublikanischen Stoffwirtschaft, in: Gleich, A. von, Leinkauf, S., Zundel, S. (Hg.): Surfen auf der Modernisierungswelle, Marburg: Metropolis-Verlag, 15-45.

Gleich, A. von, 1999, Vorsorgeprinzip, in: Bröchler, S., Simonis, G., Sundermann, S. (Hg.): Handbuch Technikfolgenabschätzung, Berlin: edition sigma, 287-293. 
Gleich, A. von, Brand, U., Stührmann, S., Gößling-Reisemann, S., Lutz-Kunisch, B., 2010a, Leitkonzepte und Gestaltungsleitbilder - Die soziale und kulturelle Dimension der Technik- und Systementwicklung, in: Fichter, K., Gleich, A. von, Pfriem, R., Siebenhüner, B. (Hg.): Theoretische Grundlagen für erfolgreiche Klimaanpassungsstrategien,Bremen, Oldenburg: nordwest2050-Bericht 1/2010,140-153.

Gleich, A. von, Brand, U., Stührmann, S., Gößling-Reisemann, S., Lutz-Kunisch, B., 2010b, Leitorientierte Technologie- und Systemgestaltung, in: Fichter, K., Gleich, A. von, Pfriem, R., Siebenhüner, B. (Hg.): Theoretische Grundlagen für erfolgreiche Klimaanpassungsstrategien,Bremen, Oldenburg: nordwest2050-Bericht 1/2010, 130-139.

Giesel, K. D., 2007, Leitbilder in den Sozialwissenschaften, Wiesbaden: VS Verlag für Sozialwissenschaften.

Hauschildt, J., Salomo, S., 2010, Innovationsmanagement. 5. Auflage, München: Vahlen.

Härtel, S., 2008, Im Gespräch mit Heinrich Bartelt, Geschäftsführer der Windpark Druiberg GmbH \& Co. KG: "Wer im Bereich der Erneuerbaren Energien investieren will, sollte nach Sachsen-Anhalt gehen". na.presseportal, verfügbar unter: http://www.presseportal.de/print.htx?nr=| 207680 (letzter Abruf Oktober 2011).

Hellige, H. D., 1991, Leitbilder und historisch-gesellschaftlicher Kontext der frühen wissenschaftlichen Konstruktionsmethodik, Bremen: artec-Paper Nr. 8.

Hellige, H. D., 1996, Technikleitbilder als Analyse-, Bewertungs- und Steuerungsinstrumente: Eine Bestandsaufnahme aus informatik- und computerhistorischer Sicht, in: Ders. (Hg.): Technikleitbilder auf dem Prüfstand. Leitbild-Assessment aus Sicht der Informatik- und Computergeschichte, Berlin: edition sigma, 15-35.

Hoffmann, K. P., 2010, Erneuerbare Energie: Das Windrad im Dorf lassen. Kommunen entdecken die erneuerbaren Energien. Erste sind schon unabhängig von den Großversorgern, Der Tagesspiegel, verfügbar unter: http://www.tagesspiegel.de/wirtschaft/ das-windrad-im-dorf-lassen/I903634.html (letzter Abruf November 2011).

Hofkirchner, W., 1996, Das Technikbild der Gestaltungs- und Wirkungsforschung, in: Böhm, H. P., Gebauer, H., Irrgang, B. (Hg.): Nachhaltigkeit als Leitbild für Technikgestaltung, Forum für interdisziplinäre Forschung, 14, 69-78.

Huber, J., 1989, Technikbilder. Weltanschauliche Weichenstellungen der Technologie- und Umweltpolitik. Opladen: Westdeutscher Verlag.

Jakobsen, I., 2008, The Road to Renewables. A case study of wind energy, local ownership and social acceptance at Samsø. University of Oslo/University of Aalborg, Innovation Systems, Social and Ecological Change, verfügbar unter: http://www.duo.uio.no/publ/tik/2008/85555/FINAL_THESIS6.pdf (letzter Abruf Oktober 2011).

Karlstetter, N., Fichter, K., Pfriem, R., 2010, Evolutorische Grundlagen, in: Fichter, K., Gleich, A. von, Pfriem, R., Siebenhüner, B. (Hg.): Theoretische Grundlagen für erfolgreiche Klimaanpassungsstrategien,Bremen, Oldenburg: nordwest2050-Bericht 1/2010, 70-100.

Kemp, R., Rotmans, J., 2005, The Management of the Co-Evolution of Technical, Environmental and Social Systems, in: Weber, M., Hemmelskamp, J. (Hg.): Towards Environmental Innovation Systems, Berlin, Heidelberg, New York: Springer, 33-55.

Klemmer, P., 1999, Innovationen und Umwelt - Fallstudien zum Anpassungsverhalten in Wirtschaft und Gesellschaft. Analytica, Berlin. 
Klemmer, P., Becker-Soest, D., Wink, R., 1998, Leitstrahlen, Leitbilder und Leitplanken - Ein Orientierungsfaden für die drei großen „L“ der Nachhaltigkeitspolitik, in: Renner, A., Hinterberger, F. (Hg.): Zukunftsfähigkeit und Neoliberalismus - Zur Vereinbarkeit von Umweltschutz und Wettbewerbswirtschaft, Baden-Baden: Nomos, 45-71.

Klemmer, P., Lehr, U., Löbbe, K., 1999, Umweltinnovationen - Anreize und Hemmnisse, Berlin: Analytica.

Knieling, J., 2006, Leitbilder und strategische Raumentwicklung - Planungstheoretische Einordnung und Diskussion der neuen Leitbilder für die deutsche Raumentwicklung, Raumforschung und Raumordnung, 6, 473-485.

Koch, R., 2006, Energieautarker Bezirk Güssing. Berichte aus Energie- und Umweltforschung, 82/2006, verfügbar unter: http://w.energiesystemederzukunft.at/edz_pdf/ 0682 energieautarker_bezirk_guessing.pdf (letzter Abruf November 2011).

Konrad, W., Nill, J., 2001,Innovationen für Nachhaltigkeit, Schriftenreihe des IÖW 157/01, Berlin.

Konrad, W., Scheer, D., 2003, Systeminnovationen: Begriff, Fallbeispiele, Nachhaltigkeitspotentiale - Zwischenergebnisse aus den Fallstudien. Experten-Workshop „Analyse und Praxis von Systeminnovationen“, verfügbar unter: http:/www.nachhaltigesbuero.de/ workshop-I/Vortrag\%20W_Konrad-D_Scheer.pdf (letzter Abruf Januar 2012).

Koolmann, S., 1992, Leitbilder der Technikentwicklung. Das Beispiel des Automobils, Frankfurt a. M., New York: Campus-Verlag.

Maaß, S., 1994, Maschine, Partner, Medium, Welt. Eine Leitbildgeschichte der Software-Ergonomie, in: Hellige, H. D. (Hg.): Leitbilder der Informatik- und Computer-Entwicklung, Bremen: artec-Paper Nr. 8, 329-342.

Mambrey, P., Paetau, M., Tepper, A., 1995, Technikentwicklung durch Leitbilder. Neue Steuerungsund Bewertungsinstrumente, Frankfurt a. M., New York: Campus Verlag.

Mano, J. M., 2010, Assessing Sustainability Claims: Key Factors in Case Studies. State University of New York New Paltz, verfügbar unter: http://www.svhe.org/files/Jo_Margaret_Mano.pdf (letzter Abruf November 2011).

Paech, N., 2005a, Nachhaltiges Wirtschaften jenseits von Innovationsorientierung und Wachstum, Marburg: Metropolis-Verlag.

Paech, N., 2005b, Nachhaltigkeit als marktliche und kulturelle Herausforderung, in: Fichter, K., Paech, N., Pfriem, R. (Hg.): Nachhaltige Zukunftsmärkte, Orientierungen für unternehmerische Innovationsprozesse im 21. Jahrhundert, Marburg:Metropolis-Verlag, 57-94.

Pflüger, J.-M., 1995, Leitbilder der Programmiersprachenentwicklung, in: Friedrich, J., Herrmann, T., Peschek, M., Rolf, A. (Hg.): Informatik und Gesellschaft, Heidelberg, Berlin, Oxford: Spektrum-Verlag, 196-210.

Rolf, A., Berger, P., Klischewski, R., Kühn, M., Maßen, A., Winter, R., 1990, Technikleitbilder und Büroarbeit zwischen Werkzeugperspektive und globaler Vernetzung, Opladen: Westdeutscher Verlag.

Schwarz, M., Linne, G., 2003, Handbuch nachhaltige Entwicklung: Wie ist nachhaltiges Wirtschaften machbar? Opladen: Leske + Budrich.

Stuiber, P. 2007, Alles grün in Güssing. Welt Online, verfügbar unter: http://www.welt.de/ wirtschaft/article736987/Alles_gruen_in_Guessing.html (letzter Abruf November 2011). 
Tepper, A., 1994, Computer für jeden und alles. Zur Rolle von Leitbildern in der Entwicklungsgeschichte des Personalcomputers, in: Hellige, H. D. (Hg.): Leitbilder der Informatik- und Computer-Entwicklung, Bremen: artec-Paper Nr. 33, 345-375.

Weber, M., Hemmelskamp, J., 2005, Towards Environmental Innovation Systems. Berlin, Heidelberg, New York: Springer.

Zieschank, R., 1999, Bodenschutzpolitik: Zur Ergänzung rechtlicher Regulierung durch Elemente kommunikativer Umweltpolitik, Berlin: FFU-Report 99-9, verfügbar unter:

http://userpage.fu-berlin.de/ffu/download/rep-99-9.PDF (letzter Abruf Januar 2012). 


\title{
Zur Governance von technologischen Innovationen im Energiesektor
}

\section{Gerhard Fuchs}

Abteilung für Organisations- und Innovationssoziologie, Universität Stuttgart

\section{Keywords}

Governance, Innovation, Energiesektor, Photovoltaik, Carbon Capture and Storage

\begin{abstract}
Die Diskussionen um den Klimawandel (zusammen mit der Liberalisierung der Energiemärkte) und der Ausstieg aus der Kernenergie setzen den Energiesektor unter einen erheblichen Innovationsdruck. Die Verbrennung fossiler Rohstoffe (Öl, Gas, Kohle) zur Stromerzeugung gilt als eine der Hauptursachen für diemenschlich verursachte Klimaveränderung. Der Beitrag setzt sich mit der Frage der Governance von Innovationen im Energiesektor auseinander und vergleicht dabei die Entwicklung von Photovoltaik in Deutschland und Japan und von $\mathrm{CO}_{2}$-Abscheidung und -Speicherung (Carbon Capture and Storage - CCS) in Norwegen und Deutschland. Der Beitrag fragt danach, welche (unterschiedlichen) Akteure und Strategien für die Entwicklung der Innovationen verantwortlich waren und wie die spezifischen Umsetzungsstrategien in Abhängigkeit von jeweils nationalspezifischen Machtkonstellationen sich gestalten.
\end{abstract}

\section{The Governance of Technological Innovations in the Energy Sector}

Keywords: Governance, innovation, energy sector, photovoltaics, carbon capture and storage

Abstract: The paper looks at the governance of new technologies in three countries being pushed as (more) climate friendly options for the generation of energy: photovoltaics (PV) in Japan and Germany, CCS (Carbon Capture and Storage) in Norway and Germany. The former is related to the development of a new climate friendly technology, the latter wants to improve the performance of coal fired power plants, in order to make them more climate friendly. The development of the markets and technologies under discussion is embedded in (four) specific strategic action fields with governance configurations aiming in different ways at promoting innovation. By using the theoretical template of strategic action fields the dynamic side of governance as well as the "who gets what" aspect is supposed to be strengthened in the analysis. 


\section{Einleitung}

In den letzten Jahren ist es der Governanceforschung in verschiedenen Bereichen gelungen, wesentliche Erkenntnisfortschritte zu erzielen (vgl. als Überblick Benz et al. 2007; Benz/Dose 2010). Unser Verständnis über die Funktionsweise von Märkten, industriellen Sektoren etc. hat sich wesentlich verbessert. Governance steht dabei für alle Formen und Mechanismen der Koordinierung zwischen mehr oder weniger autonomen Akteuren, deren Handlungen interdependent sind, sich also wechselseitig beeinträchtigen oder unterstützen können (Benz et al. 2007:9). Die Überlegungen zur Bedeutung von Governance sind institutionalistisch orientiert und fragen nach der Beschaffenheit von spezifischen Regelungsstrukturen (Mayntz 2004). Die Forschung hat sich damit auf die eher statisch-strukturellen Aspekte von Governance konzentriert. Nahezu die gesamte einschlägige Forschung fokussiert sich auf das interne Funktionieren von Governancestrukturen und unterstellt, dass diese weitgehend selbstbezogen operieren.

Mindestens ebenso bedeutsam ist aber die Frage nachder Veränderung von existierenden Strukturen. Es ist hinreichend diskutiert worden, dass sich Strukturen, Institutionen ebenso wie Organisationenals beharrlich erweisen, Pfadabhängigkeiten eine wichtige Rolle spielen und Veränderungen nur widerwillig vollzogen werden. Die institutionalistische und evolutionstheoretisch informierte Forschung hat immer wieder zu zeigen versucht, dass Veränderungen, insbesondere Veränderungen grundsätzlicher Natur, in erster Linie durch externe Anforderungen (Meyer/Rowan 1977) bzw. Krisen und Schocks initiiert werden und nicht durch die etablierten Hauptakteure in Organisationen, Sektoren oder Politikfeldern vorangetrieben werden.

Ein gutes Beispiel hierfür ist der Energiesektor. Die Entwicklung des Energiesektors war immer stark beeinflusst gewesen von einer kleinen Gruppe industrieller Akteure sowie politischen und regulatorischen Entscheidungen (Viktor 2002). In vielen Ländern sind Entscheidungen zum Einsatz bestimmter Technologien (z. B. Kernkraft, Erneuerbare Energien) begleitet gewesen von Enqueten, Berichten, Szenarien und Folgenabschätzungen. Wesentliche Anstöße für Veränderungen im Energiesektor sind dabei aber immer wieder durch externe Einflüsse zustande gekommen: der ÖlpreisSchock Mitte der 70er Jahre, die Tschernobyl-Katastrophe und die darauf aufbauende breite Stimmung in der deutschen Bevölkerung gegen einen weiteren Ausbau der Atomkraft, die durch die Europäische Kommission vorangetriebene Liberalisierung der Energiemärkte und schließlich Fukushima.

Diese externen Ereignisse können unter bestimmten, genauer zu analysierenden Bedingungen zu Veränderungen von Governancestrukturen beitragen, ebenso wie unter „Normalbedingungen“ davon auszugehen ist, dass externe Herausforderungen von der Akteuren erst einmal innerhalb und mit den existierenden Strukturen abgearbeitet werden. Wir nehmen an, dass die Veränderung von Governance weniger durch die Krise selbst, technologische Entwicklungen oder einen externen Schock per se herbeigeführt wird, sondern durch Prozesse in denen sich Alternativen zur herrschenden Problembearbeitung entwickeln und die durch eine Veränderung in gesellschaftlichen Kräfteverhältnissen getragen wird.Prozesse der Veränderungen von Governance drehen sich um die Frage, wer, was unter welchen Bedingungen bekommt. Eine theoretische Erklärungsperspektive, die uns erlaubt, solche Prozesse besser zu verstehen, ist die Theorie der strategischen Handlungsfelder von Neil Fligstein (Fligstein/McAdam 2011), die wir im Folgenden als Grundlage für unsere Diskussion heranziehen wollen. Kategorial lassen sich unter einer dynamischen Perspektive die folgenden Alternativen für die Entwicklung von Governance als Antwort auf neue Herausforderungen vorstellen: 
1. Eine Wiedereinsetzung bzw. Fortführung der alten Ordnung mit einigen Anpassungen

2. Zusammenbruch der Governanceund Auflösung in unorganisierten sozialen Raum

3. Weitere Ausdifferenzierung der Governancestruktur

4. Entstehung einer neuen Governancestruktur (Transformation).

Es ist davon auszugehen, dass die in dem jeweiligen strategischen Handlungsfeld dominierenden Akteure versuchen werden, den status quo und die ihn repräsentierende Governance zu verteidigen. Herausforderungen werden in erster Linie von Akteuren kommen, die eine schwächere Position innerhalb des Feldes haben oder bislang keine Rolle im Feld spielten. Sie werden sich feldexterner Entwicklungen oder Ereignisse bedienen, um möglicherweise neue Regeln, im Endeffekt eine neue Feldordnung durchzusetzen.

\section{Neue Technologien, Governance und der Energiesektor}

Das Energiesystem steht, wie oben bereits angedeutet, weltweit unter großem Anpassungsdruck. Große Energieinfrastrukturen sind die Voraussetzungen für wirtschaftliche Entwicklung. Allerdings wird die dominierende Art der Energiegewinnung aus fossilen Brennstoffen (Kohle, Öl, Gas) auch verantwortlich gemacht für die durch Menschen verursachte Klimaveränderung. Alternative Arten der Energiegewinnung sollen entwickelt und umgesetzt werden bzw. der Einsatz fossiler Brennstoffe klimafreundlicher gestaltet werden. Als Beispiel für letzteres werden wir die Diskussion um die so genannte Carbon Dioxide Capture and Storage (CCS)-Technologie vorstellen, die den Betrieb von kohlebetriebenen Kraftwerken klimafreundlicher gestalten soll. Eine Herausforderung für die etablierten Governancestrukturen des Energiesektors kam und kommt von Seiten der Erneuerbaren Energien. Die traditionelle Form der Elektrizitätsgewinnung baut auf zentralisierten Strukturen mit Großkraftwerken, die nur von wenigen potenten Unternehmen gebaut und betrieben werden können. Erneuerbare Energien sind im Gegensatz dazu nicht nur mit dem Anspruch angetreten, klimaschädliche Formen der Energiegewinnung abzulösen, sondern auch eine dezentralisierte Form der Energiegewinnung zu forcieren, an der sowohl auf Produzenten- wie Konsumentenseite neue Akteure beteiligt sind. Eine gänzlich andere Form der Governance ist zumindest theoretisch möglich.

\section{Die Förderung von CCS in Deutschland und Norwegen}

Am Beispiel von CCS lässt sich zeigen, wie Governance von inkrementellen Innovationen im Energiesektor ausgestaltet ist und wie unterschiedliche Akteure und Strukturen im selben Sektor zu einer unterschiedlichen Performanz beitragen, einer blockierten Entwicklung in Deutschland auf der einen Seite und einer von einem breiten Konsens getragene Situation in Norwegen. 


\section{I CCS in Norwegen}

Für die Stromgewinnung wird in Norwegen traditionell und fast exklusiv die Wasserkraft genutzt. Die heimischen Öl- und Gasvorräte werden primär exportiert. Die Diskussion um CCS wurde auch hier von Akteuren vorangetrieben, die zunächst keine etablierte Position im Energieversorgungssystem hatten. Treibende Akteure für die Entwicklung der Technologie und die Etablierung einer Governance Struktur für CCS in Norwegen waren zunächst Norwegens größter Ölkonzern Statoil sowie die Forschungseinrichtung SINTEF und die Technische Universität von Trondheim NTNU. Bereits in den 1980er Jahren wurde die Idee einer Abscheidung und Speicherung von $\mathrm{CO}_{2}$ entwickelt. Zur selben Zeit saß Norwegens Ministerpräsidentin Gro Harlem Brundtland der World Commission on Environment and Development der Vereinten Nationen vor. Unter ihrer Führung wurde ein umfassender Bericht zur Nachhaltigen Entwicklung verfasst. In der Folge führte Brundtland 1991 in Norwegen für verschiedene fossile Brennstoffe und Sektoren eine $\mathrm{CO}_{2}$-Steuer ein. Diese Steuer war dann in den 1990er Jahren mit ausschlaggebend, die Pläne der Abscheidung und Injektion von $\mathrm{CO}_{2}$ in Öl- und Gasfelder zunächst als Forschungsaktivitäten und zunehmend auch als kommerzielle Projekte durchzuführen. Die Öl- und Gasindustrie hatte insbesondere Interesse in einem der CCS Technologie verwandten Bereich: Mit sogenannten EOR (Enhanced Oil Recovery) oder EGR (Enhanced Gas Recovery)-Verfahren wird in off shore Öl- und Gasfeldern $\mathrm{CO}_{2}$ zur verbesserten Öl- und Gasausbeute eingeleitet. Schnell vergrößerte sich das Akteursnetzwerk um weitere Forschungspartner. Zunächst kam Norwegens größter Anlagenbauer, Kvaerner hinzu. Im Laufe intensiver Forschungsprojekte schlossen sich weitere Akteure an, insbesondere internationale Ölkonzerne. Treibende Kraft in Norwegen war die Öl- und Gasindustrie, die begann ihre F\&E-Aktivitäten sowie Partnerschaften mit wissenschaftlichen Einrichtungen mit der Injektion und Speicherung von $\mathrm{CO}_{2}$ in die fast leer geförderten Öl- und Gasfelder. Ab 1996 startete Statoil mit der ersten kommerziellen Nutzung im Gasfeld Sleipner West in der Nordsee.

Der Anlagenbauer Kvaerner begann parallel seit 1992 mit F\&E-Programmen zur Abscheidung. Dabei konzentrierte man sich auf das sogenannte Post Combustion Verfahren. Ab 1997 erhielten Forschungsaktivitäten zu CCS auch öffentliche Forschungsgelder aus dem KLIMATEK Programm der norwegischen Regierung. Nachdem Kvaerner mit der ersten Pilotanlage eines $\mathrm{CO}_{2}$-Abscheiders erfolgreich gestartet hatte, investierte auch Norwegens zweiter großer Technologiehersteller, Aker, in $\mathrm{F} \& \mathrm{E}$ zur $\mathrm{CO}_{2}$-Abscheidung.

Von gesellschaftlicher Bedeutung und von Interesse für die norwegische Elektrizitätsversorgung wurde CCS im Folgenden besonders aufgrund des zunehmenden Strombedarfs, der wegen Umweltschutzbedenken nicht mehr durch den Zubau neuer Wasserkraftwerke gedeckt werden sollte. Nachdem der norwegische Energieversorger Naturkraft zwei Konzessionen für den Bau von Gaskraftwerken erhalten hatte, brach eine umweltpolitische Debatte los, da Gaskraftwerke (wenn auch nicht im selben Ausmaß wie Kohlekraftwerke) $\mathrm{CO}_{2}$-Emissionen verursachen. Einflussreiche Umweltorganisationen brachten in diesem Zusammenhang die CCS-Technologie als mögliche Option ins Spiel, einerseits um dem gestiegenen Strombedarf gerecht zu werden und gleichzeitig dem inzwischen politischen (auch durch das Kyoto Protokoll auf internationaler Ebene verankerten) Ziel einer $\mathrm{CO}_{2}$-Reduktion Rechnungzu tragen. Da die privaten F\&E-Aktivitäten, die norwegische Politik sowie die geologischen Speicherpotenziale immer größere, nun auch von der EU (obwohl Norwegen kein EU-Mitglied ist) ko-finanzierte Forschungsverbünde aktiv werden lassen, verbuchte CCS einen immensen Legitimationsschub in der breiten Bevölkerung, aber auch unter den meisten NGOs. Die ursprüngliche Debatte um den Bau oder Nicht-Bau zweier Gaskraftwerke hatte sich schon bald in eine Debatte verwandelt, ob die Gaskraftwerke mit oder ohne CCS-Technologie gebaut werden sollten (vgl. van Alphen et al 2009: 47). Seit 2001 ist die norwegische Politik von der Leitlinie geprägt, keine weiteren Konzessionen für Kraftwerke ohne CCS zu erteilen. 
Zusammenfassend lässt sich sagen, dass CCS in Norwegen von einer breiten Koalition von Akteuren aus Staat, Wirtschaft und Industrie vorangetrieben wurde, die auf eine vorhandene Unterstützung in der Bevölkerung bauen konnten. Die Entwicklung der Technologie hat zu keinem disruptiven Wandel geführt, sondern war daran orientiert, vorhandene Akteurskoalitionen auf weitgehend konsensuellem Weg zu verbreitern.

\subsection{CCS in Deutschland}

Eine Betrachtung der Governance von Innovationen für CCS in Deutschland zeigt eine von der Situation Norwegens signifikant unterschiedliche Ausgangssituation. Nach wie vor spielt Kohle für die Stromerzeugung in Deutschland eine bedeutende Rolle: Der Anteil der Braunkohle liegt bei $24 \%$, der Anteil der Steinkohle bei $18 \%$ (UBA 2011). Die eingesetzte Braunkohle stammt zu fast $100 \%$ aus inländischen Quellen und ist der einzige wettbewerbsfähige heimische Energierohstoff von Relevanz. Nach einer Phase der Stagnation dominieren Kohlekraftwerke in den letzten Jahren auch wieder den Markt, d. h. laufende und geplante Bauprojekte sind vorrangig Kohlekraftwerke (vgl. Pahle 2010). In ihrer Rolle als Kunden von Kraftwerkstechnologien haben die deutschen Energieversorger ein großes Interesse an technologischen Innovationen, einerseits für verbesserte herkömmliche Technologien, die sich z. B. in verbesserten Wirkungsgraden zeigen, andererseits auch an der CCS-Technologie, die $\mathrm{CO}_{2}$-Emissionen auf ein sehr niedriges Niveau senken würde. Da sich Deutschland zu einer ehrgeizigen Klimapolitik ( $40 \%-\mathrm{CO}_{2}$-Reduktionsziel der Bundesregierung) verpflichtet hat, nimmt auch von Seiten der nationalen Politik der Druck auf die Kohlekraftwerksbetreiber zu, ihre Emissionen zu senken.

Die große Bedeutung, die Kohle für die deutsche Stromerzeugung besitzt, spiegelt sich darin wider, dass Deutschland als weltweit führend bei der Entwicklung von Kohlekraftwerkstechnologien gilt (Weimer-Jehle/Wassermann/Fuchs 2010). Innovationsaktivitäten im Bereich Kohlekraftwerke und CCS werden in Deutschland von einer überschaubaren Anzahl, vorrangig großer Akteure vorangetrieben. Dies sind multinationale Unternehmen wie Siemens, Alstom und Hitachi Power Europe, die als bedeutende Kraftwerksbauer technisch hochentwickelte Komponenten wie Turbinen, Kessel und Generatoren fertigen, sowohl für deutsche Nachfrager als auch für den Weltmarkt. Innovationen finden vorrangig in Clustern von Forschungsnetzwerken zwischen Forschungseinrichtungen, wie z. B. dem Forschungszentrum Jülich, großen Universitätsinstituten, den F\&E-Abteilungen der Kraftwerkshersteller sowie auch den F\&E-Abteilungen der Kunden, i.d.R. der vier großen Energiekonzerne RWE, E.ON, Vattenfall und EnBW statt (vgl. Rogge/Hoffmann 2009: 7). Treibende Akteure bei der Entwicklung und Verbreitung von CCS sind daher in Deutschland zum einen die Kraftwerkshersteller, als Unterstützter tritt auch die heimische Braunkohleindustrie auf, und natürlich die großen Energieversorgungskonzerne, die zum einen die Mehrzahl der in Deutschland laufenden Kohlekraftwerke betreiben und die zum anderen den zunehmend drohenden Zertifikathandel und die daraus für sie entstehenden hohen Kosten fürchten (vgl. Rogge/Hoffmann 2009). Aufgrund der industriepolitischen Bedeutung für die Kraftwerkshersteller wurden in Deutschland zunächst Forschungsaktivitäten im Bereich der $\mathrm{CO}_{2}$-Abscheidung von Seiten der Politik unterstützt und vorangetrieben. Hierbei war das Bundesministerium für Wirtschaft und Technologie (BMWi) federführend: Im Rahmen der COORETEC-Initiative zur Förderung von Forschung und Entwicklung zukunftsfähiger Kraftwerke mit fossilen Brennstoffen wurden Forschungsprojekte und Pilotanlagen zur Abscheidung von $\mathrm{CO}_{2}$ finanziert. Am Standort Schwarze Pumpe in Brandenburg, einem großen und traditionellen Braunkohlebergbaugebiet, befindet sich die weltweit erste Versuchsanlage für ein $\mathrm{CO}_{2}$-armes Braunkohlekraftwerk nach dem Oxyfuel-Verfahren. Die Pilotanlage war 2008 vom Energiekonzern Vattenfall in Betrieb genommen worden und dient der Erforschung und Weiterentwicklung des Oxyfuel-Prozesses, um die Technologie zur Marktreife zu führen. Parallel entwickelt Vattenfall ein 300 MW-Demonstrationskraftwerk, das in den nächsten Jahren am Standort 
Jänschwalde(ebenfalls Brandenburg) in Betrieb gehen soll. Anders als in Norwegen kommen die treibenden Kräfte und Unterstützerkoalitionen für CCS aus dem Kraftwerks- und Bergbausektor. Hier lassen sich die inkrementellen Innovationsprozesse anhand der Aktivitäten der traditionellen Akteure und Netzwerke nachzeichnen.

Es stellte sich aber schnell heraus, dass die zweite Stufe der CCS-Technologie (d. h. die Suche nach geeigneten Lagerstätten für das abgeschiedene $\mathrm{CO}_{2}$ ), die auf keine traditionelle Governancestrukturen und Unterstützerkoalitionen zurückgreifen konnte, bei ihrer kommerziellen Erprobung auf Akzeptanzprobleme stieß, die den Innovationsprozess zum Stoppen brachten. So gibt es massive Widerstände gegen die Exploration geeigneter Lagerstätten. Verschiedene Bürgerinitiativen gründeten sich, die Unterstützung durch Umweltverbände, aber auch von Verbänden, wie dem Bauernverband und dem Verband der Wasserwirtschaft erhalten haben (Schulz/Scheer/Wassermann 2010). Nach massiven Bürgerprotesten und einer als Reaktion darauf letztlich ablehnenden Haltung verschiedener Landesregierungen, allen voran der Regierung von Schleswig-Holstein, scheiterte zunächst die Verabschiedung eines CCS-Gesetzes auf Bundesebene. Als Folge stoppte RWE den Bau des Demonstrationskraftwerks in Hürth. Zuvor war RWE bereits bei der Bewerbung um EUFördermittel für das Projekt gescheitert, da man aufgrund der Widerstände, denen man sich bei der Suche nach möglichen Lagerstätten ausgesetzt sah auch keine Speicherpotenziale nachweisen konnte. Die bislang in Deutschland einzige Genehmigung zur Exploration eines potenziellen kommerziellen $\mathrm{CO}_{2}$-Speichers, nämlich für zwei Standorte in Brandenburg, erfolgte durch das brandenburgische Landesamt für Bergbau, Geologie und Rohstoffe (LBGR) auf Grundlage bergrechtlicher Regelungen und Bewertungen. Das LBGR hatte bei der Genehmigung allerdings zunächst auf das noch ausstehende CCS-Gesetz des Bundes verwiesen. Nachdem dieses im Sommer 2009 (insbesondere am Widerstand Schleswig-Holsteins) im Bundesrat gescheitert war, verfügte das Land Brandenburg im Dezember 2009, dass eine ,Übergangslösung“ bis zur Verabschiedung eines nationalen Gesetzes angemessen sei. Inzwischen wurde ein neuer Gesetzesvorschlag erarbeitet, der nach zähen Diskussionen im April 2011 zunächst vom Kabinett verabschiedet wurde. Aber von Seiten der CCS-Unterstützer wurde dem Entwurf bereits vorgeworfen, eher ein CCS-"Verhinderungsgesetz“ zu sein, da den Bundesländern zu viel Mitsprache eingeräumt wurde. Am 23. September stimmte der Bundesrat gegen das Gesetz. So ist die Zukunft dieser Technologie in Deutschland momentan sehr unsicher.

Ähnlich wie in Norwegen sind die etablierten industriellen Akteure, die sich mit der Forschung vernetzten, an einer Entwicklung der CCS-Technologie interessiert. Auch der Staat ist ähnlich wie Norwegen klimapolitisch stark engagiert und befürwortet aus diesem Grunde CCS. Hier enden aber die Gemeinsamkeiten. CCS in Deutschland kann nicht auf einen breiten sozialen Konsens bauen und die die Technologie unterstützenden Akteure sind im Wesentlichen die etablierten Hauptakteure des Energiesystems. Die Aktivitäten von Bürgerinitiativen, NGOs etc. haben dazu geführt, dass sich eine einflussreiche Gegenbewegung organisiert hat und die Politik dazu veranlasste, bislang keine belastbare Regulierung für den Einsatz von CCS durchzusetzen. Ob CCS in Deutschland eine Zukunft hat, ist damit mehr als offen. 


\section{Die Förderung von Photovoltaik in Deutschland und Japan}

Im Gegensatz zu den eher inkrementellen Innovationen im Bereich klimafreundlicher Kohlekrafttechnologien sind an der Entwicklung und Diffusion erneuerbarer Energien insbesondere in Deutschland eine Vielzahl neuerAkteure beteiligt, wie neue Hersteller, Stromhändler sowie Betreiber dezentraler Anlagen. Die klima- und umweltpolitischen Herausforderungen haben darüber hinaus zu einem Gefüge von politischen Eingriffen in den Energiemarkt sowie in den sich neu entwickelnden Energiemix geführt. Neue Steuerungsinstrumente wurden entwickelt und es sind zumindest in Deutschland neue Akteurskonstellationen zu beobachten, die wiederum zu neuen Governancestrukturen geführt haben.

\section{I Photovoltaik (PV)-Entwicklung in Japan}

Die Anfänge der PV-Forschung gehen in Japan bis in die 1960er Jahre zurück. Der Konzern Sharp war damals mit der Entwicklung von Solarzellen für die Weltraumforschung befasst. Mit der Ölkrise in den 1970er Jahren, die Japan aufgrund seiner Importabhängigkeit besonders schmerzlich traf, startete die japanische Regierung 1973 ein erstes öffentliches Programm, das „Sunshine Program“, um die Importabhängigkeit zu reduzieren. Ein kleiner Anteil, 6 Mio US-Dollar, war darin auch zur Förderung der PV-Forschung für terrestrische Anwendungen vorgesehen. 1978 dehnte Japan dann seine Forschungsanstrengungen im Bereich PV aus.

Im Zentrum des japanischen Innovationssystems stehen große, integrierte Firmen, die inkrementelle Weiterentwicklungen von Produkten und Prozessen durchführen. Zweiter wichtiger Akteur für die Governance von Innovationen in Japan ist der Staat. Er ist deutlich aktiver und lenkender alsz. B. dies in Deutschland der Fall ist: „Japan and Germany clearly display different social systems of innovation, and this is why these countries showed contrasting patterns of evolution during the last quarter of the twentieth century" (Boyer 2003: 148). Vogel (2006) weist auf bedeutende Unterschiede zwischen der deutschen und der japanischen Innovationspolitik hin: „The German government merely facilitates private-sector coordination, whereas the Japanese government organizes and guides the private sector more directly. The German government has codified its economic model into law, whereas the Japanese model relies more on informal norms and standard practices." (Vogel 2006: 308).

Dass der japanische Staat mit unterschiedlichen Maßnahmen und Strategien aktiv ins Geschehen eingreift, zeigt sich sowohl für den Energiesektor als Ganzes, als auch für den PV-Bereich im Speziellen. Nach dem zweiten Ölpreisschock 1979 gründete die Regierung 1980 die New Energy Development Organization (NEDO) mit dem Ziel insbesondere die Erdölabhängigkeit des Landes zu überwinden. NEDO ist dem Ministerium für internationalen Handel und Industrie (MITI) angegliedert, das auch für Energiefragen zuständig ist. NEDO übernahm die Rolle des MITI für den PV-Bereich. Seit 1988 heißt die Organisation „New Energyand Industrial Technology Development Organization" und betont damit noch stärker ihre Industrieverantwortung (vgl. Ristau 1998: 81). Mitarbeiter der NEDO kamen sowohl von staatlichen Einrichtungen wie aus der Industrie. So spielte u. a. auch der Energiekonzern Tokyo Electric Power Company eine wichtige Rolle bei der Ausgestaltung der Energiekonzepte und Strategien der Organisation.

In den 1980er Jahren übernahm NEDO für den PV Bereich zwei zentrale Aufgaben: Zum einen wurden Forschungsprojekte zur Verbesserung des Wirkungsgrades von Solarzellen finanziert. Zum anderen trat NEDO als wichtigster Nachfrager für die industriell hergestellten Solarzellen in Er- 
scheinung. Da in den 1980er Jahren weder ein heimischer noch ein Exportmarkt für PV-Anwendungen existierte, war die öffentlich generierte Nachfrage entscheidend für die japanische Industrie, sich eine weltweit führende Rolle in der PV-Technologie zu erarbeiten. Sukzessive etablierte sich ein Weltmarkt, den Japan rasch bedienen und entsprechend seine Marktanteile steigern konnte: „Besaßen die Japaner 1983 am weltweiten Absatz von Modulen einen Anteil von 23 Prozent, betrug dieser nach einer Erhebung der europäischen Solarvereinigung Eurosol zwei Jahre später schon 45 Prozent“" (Ristau 1998: 81).

Die Stärke des japanischen Innovationssystems, die sich auch für den PV-Bereich zeigt, liegt nicht nur in der korporativen Forschungsförderung, sondern auch in den gewählten politischen Instrumenten zur Technologiediffusion (wie z. B. der Finanzierung von Demonstrationsanlagen und Marktanreizprogrammen) begründet. Um der Industrie Anreize zu geben, ihre Produktionskapazitäten auszudehnen, legte das MITI bereits 1994 das sogenannte 70.000 Dächer Programm (,Monitoring Program for Residential PV Systems“) auf (vgl. Shum/Watanabe 2009: 3536). Durchgeführt wurde das Programm von der New Energy Foundation (NEF). Darin übernahm die Regierung 50 \% der Installationskosten privater Haushalte, aber auch Firmen konnten Subventionen für die Installation von PV-Anlagen beantragen. Finanziert wurde das Programm durch einen Aufpreis aufdie Stromtarife. Die Energieversorger wurden darüber hinaus dazu verpflichtet, überschüssigen PV-Strom zum Handelspreis zu erwerben. 2003 erfolgte eine Anpassung des Programms, nun wurden nur noch 15 \% der Kosten vom Staat übernommen. 1997 wurde das Neue Energien Gesetz (,Law on Special Measures to Promote Use of New Energies") verabschiedet. Es beinhaltete eine Mischung aus Subventionen und anderen Policies um die Verbreitung von PV (und anderen erneuerbaren Energien) zu befördern. Darin waren auch explizite Zielvorgaben formuliert: Gegenüber einer Verbreitung von PV 1996 von 57 MW wurde für 2010 das Ziel von 5000 MW vorgegeben (,Long-term Energy Supply/Demand Outlook"). Weitere Gesetze mit Zielvorgaben zur Verbreitung von PV folgten, ebenso wie eine Bandbreite an Projekten, die z. B. auch die öffentliche Nachfrage nach PV-Anlagen befördern sollten. Das Bildungsministerium legte ein Eco-School Project auf, das Infrastrukturministerium das Green Government Office Project und zwischen 1992 und 1998 wurde ein Field Test Project on Photovoltaic Power Generation for Public Facilities durchgeführt, das 1998 durch das Field Test Project on Photovoltaic Power Generation for Industrial and Other Applications abgelöst wurde (vgl. Anderson et al. 2008: 26).

Die Ausgaben auf staatlicher Seite in den 1990er Jahren zur Förderung von PV waren deutlich höher als in allen anderen Ländern. Der Haushalt 1997 sah Ausgaben von 150 Mio. Euro vor. Davon entfiel etwas weniger als die Hälfte auf F\&E, der größere Anteil wurde für die Nachfrage förderung verwandt (vgl. Ristau 1998: 92). Seit 1997 wurden die Subventionen mit einem weiteren Programm weiter ausgedehnt: Mit dem „Program for the Development of the Infrastructure for the Introduction of Residential PV Systems“" stiegen die Subventionen zwischen 1997 und 2001 von 11,11 Mrd. Yen auf 23,5 Mrd. Yen an (vgl. Shum/Watanabe 2009: 3536).

Die in Japan verbreitete Technologie gleicht einem standardisierten Massenprodukt ohne nennenswerte kundenspezifische Anpassung (vgl. Shum/Watanabe 2009: 3540). Der in Japan traditionell vorherrschende vertikale integrierte Innovationsprozess lässt sich auch für PV-Anlagen und sogar ihre letztliche Anwendung, dem Auf- und Anbau durch Handwerker und Architekten finden. Shum und Watanabe greifen in ihrer Beschreibung der japanischen Governance von PV-Innovationen auf das Bild einer ,geschlossenen“ Entwicklung zurück (vgl. Shum/Watanabe 2009: 3540).

Die Entwicklung der PV in Japan entspricht dem vergleichbarer anderer japanischer Innovationsprozesse. Im Mittelpunkt steht die Kooperation zwischen den Hauptakteuren aus Staat und Industrie mit dem Ziel weltmarktfähige Produkte zu erzeugen, die es der heimischen Industrie erlauben sollte, entsprechende Exporterfolge zu erzielen. Für die Umsetzung wurden die etablierten Kooperationsformen benutzt, um inkrementell und kontinuierlich die Innovation voranzutreiben. Trotz der technologischen und industriellen Vorreiterposition, die Japan über eine lange Zeit behaupten 
konnte, ist die Installation von PV-Anlagen in Japan allerdings wenig beeindruckend. Hier spielt eine Rolle, dass es trotz vieler staatlicher Maßnahmen nicht gelang einen Markt für PV-Anlagen in Japan aufzubauen. Die etablierten Akteure, die die PV-Entwicklung vorantrieben, hatten eher die Exporterfolge im Auge als eine signifikante Änderung des Energiemixes, der sich auf die Kernenergie als Hauptbestandteil fokussierte. Die Zusammensetzung der für die Entwicklungen im Energiesektor verantwortlichen Hauptakteure blieb unverändert und damit waren auch keine grundlegenden Veränderungen erwartbar.

\subsection{Beispiel Deutschland}

Für den deutschen PV-Sektor lassen sich dagegen radikale Innovationen und deutliche Brüche bzw. Veränderungen in der Governance feststellen. Während sich in den bisher diskutierten Beispielen eine kontinuierliche staatliche Förderpolitik beobachten ließ, die auf der Kooperation der Hauptakteure aus Staat und Industrie beruhte, sind für den deutschen Fall lange Zeit eher bescheidene staatliche Unterstützungsbemühungen und bis in die Gegenwart wechselnde Prioritäten feststellbar. Im Gegensatz zu Norwegen oder Japan oder der CCS-Entwicklung in Deutschland, waren für die Aufrechterhaltung des Interesses an PV insbesondere nicht-konventionelle Akteure (z. B. so genannte soziale Bewegungen) verantwortlich.

Ausgelöst durch die Ölkrise wurden in Deutschland in den 1970er Jahren die ersten politischen Programme zur F\&E-Förderung der Photovoltaik initiiert. Zu diesem Zeitpunkt lag die politische Zuständigkeit für die Photovoltaik beim Forschungsministerium (BMFT). Als die Ölpreise dann jedoch wieder sanken und mit Beginn der CDU/FDP-Koalition unter Helmut Kohl, wurden politische Programme zur Förderung der Photovoltaik deutlich zurückgefahren. Trotzdem hatte die frühe Forschungsförderung in den 1980er Jahren bereits erste Früchte getragen: zum einen hatten es die beiden industriellen Hauptempfänger der öffentlichen PV-Förderung, AEG-Telefunken und Siemens-Solar geschafft, internationale Konkurrenzfähigkeit zu erlangen (vgl. Ristau 1998: 46). Zum anderen konnte sich die deutsche PV-Forschung etablieren und gemeinsam mit Japan und den USA eine internationale Spitzenstellung einnehmen. Hierbei war ausschlaggebend gewesen, dass die Forschungsgelder sehr breit verteilt, in unterschiedliche Technologien und Verfahren flossen, und sowohl universitäre Institute als auch insbesondere angewandte Forschungszentren, wie etwa das 1982 gegründeten Fraunhofer Institut für Solare Energiesysteme (ISE) in Freiburg davon profitierten. Auf diese Weise wurden Forschungsprojekte zu einer Bandbreite unterschiedlicher (konkurrierender) Technologien finanziert, die nicht von vornherein bezüglich ihrer Zukunftsfähigkeit und ihres Marktpotenzials von politischer Seite aus bewertet worden waren. Trotz der erfolgreichen Anfängekann die Entwicklung in den 1980er Jahren noch nicht als erfolgreich bezeichnet werden. Im Gegenteil, aufgrund der fehlenden inländischen Nachfrage, wurden zwar, erfolgreich' Photovoltaiktechnologien fast bis zur Marktreife entwickelt und produziert, trafen dann jedoch auf kein gesellschaftliches und politisches Interesse und es kam zu keiner Anwendung der neuen Technik. Die Entwicklung der Technologie wurde vielmehr durch etablierte Marktakteure des Energiesektors, nämlich die Energieversorgungsunternehmen bedroht, die mit besten Kontakten zu den Entscheidungsträgern und Verwaltungsebenen in den Ministerien ausgestattet waren und eine Förderung der PV zu verhindern suchten. Hinzu kamen Kompetenzstreitigkeiten bzw. eine unglückliche Verteilung der Zuständigkeiten auf verschiedene Ministerien, die eine Innovationen befördernde Politik zu diesem Zeitpunkt eher behinderte. Traditionell werden Markteinführungsprogramme vom Wirtschaftsministerium (BMWi), F\&E vom Forschungsministerium (BMFT) finanziert (vgl. Ristau 1998: 44ff.). Das Wirtschaftsministerium galt und gilt als Gegner einer PV-Förderung. 
Dann jedoch bekam die nur sehr schwache, hauptsächlich aus PV-Forschern und den bis dahin nur wenigen in der 1975 gegründeten Deutschen Gesellschaft für Sonnenenergie (DGS) Aktiven bestehende PV-Koalition überraschend Unterstützung: Externe Ereignisse führten dazu, dass der Bekanntheitsgrad von Photovoltaik sich rasch erhöhte und sich ihre Akzeptanz als alternative Energieressource schnell verbreiterte. So veränderte der Reaktorunfall von Tschernobyl im Jahre 1986 die öffentliche Meinung hinsichtlich der Akzeptanz der Kernenergie massiv und bot damit die Möglichkeit, eine generelle gesellschaftliche Diskussion über die Zukunft des Energiesystems und die Möglichkeiten einer Transformation anzustoßen. Innerhalb von zwei Jahren stieg die Ablehnung der Kernenergie in der deutschen Bevölkerung von 50 \% auf über 70 \% (vgl. Jahn 1992). Die PVWissenschaftler schafften es zu diesem Zeitpunkt, PV als Alternative zur Atomenergie, als ein wichtiges Element für die Transformation des Energiesektors und als saubere, daher umweltfreundliche Energiequelle zu lancieren. Damit trafen sie die unterschiedlichen Interessen der in den verschiedenen Umweltgruppen und Antiatomkraftbewegungen Engagierten. Im Vergleich zu anderen europäischen Ländern waren die Umweltbewegungen, die breite Ablehnung der Kernenergie nach dem Tschernobyl-Unfall und die Partei „Die Grünen“ in Deutschland besonders stark. Aus diesem Grund fühlten sich politische Entscheidungsträger zu einem vergleichsweise frühen Zeitpunkt dazu veranlasst, die Photovoltaik mit einem Markteinführungsprogramm zumindest symbolisch zu unterstützen. 1991 startete das 1000-Dächer-Programm. Finanziert von der Kreditanstalt für Wiederaufbau (KfW)bot es verbilligte Kredite für private Haushalte, die gleichzeitig Interesse zeigten, an einer groß angelegten Testphase für netzgekoppelte Photovoltaikanwendungen teilzunehmen. NGOs, wie die DGS, der Solarenergie-Förderverein oder Eurosolar nutzten diese Situation, um Einfluss auf die Politik auszuüben. Sie entwickelten Einspeisungs- und Finanzierungsmodelle und bewarben sie. Die 1990er Jahre wurden im Folgenden insbesondere durch institutionelle Neuerungen auf globaler und EU-Ebene geprägt, die Einfluss auf die Photovoltaik-Innovationspolitik in Deutschland nahmen. Die von der EU geforderten Deregulierungen auf dem Strommarkt einerseits und eine zunehmende Wahrnehmung durch die Bedrohung eines Klimawandels andererseits, die etwa in dem 1997 unterzeichneten Kyoto-Protokoll ihren Ausdruck fand, führten zur weiteren Formierung und Organisierung von Befürwortern der Photovoltaik und zu einer zunehmend positiveren Einschätzung der Photovoltaik als Lösung des Klimaproblems und zu einer Verschärfung der Diskussion um neue, verbesserte politische Instrumente.

Da das Auslaufen des 1000-Dächer-Programms einen starken Rückgang der Nachfrage verursacht hatte und die sinkenden Strompreise PV unrentabel machten, sah es ab Mitte der 1990er Jahre zunächst so aus, als hätten Photovoltaik-Anwendungen in Deutschland keine Zukunft mehr. Der Markt brach weg, trotz einer breiten Zustimmung in der Bevölkerung und noch schlimmer: Die sich langsam entwickelnde Industrie drohte nun damit, ins Ausland abzuwandern und Deutschland den Rücken zu kehren. Ohne eine langfristige, umfassende politische Unterstützung gäbe es in Deutschland keine Nachfrage nach Photovoltaikanlagen, so die Argumentation der Hersteller. Dies war das Signal für NGOs erneut initiativ tätig zu werden. Greenpeace gab bei der Ludwig-Bölkow-Stiftung eine Studie über die Errichtung einer Solarfabrik in Auftrag, die zu dem Ergebnis kam, dass eine Markteinführung von Solarstromanlagen am Standort Deutschland rentabel sein könnte (vgl. Welt online 1995). Durch Skaleneffekte und einer entsprechenden Automatisierung im Produktionsprozess ließen sich die Preise von Photovoltaikanlagen um 40 \% reduzieren. Dies ließe sich bereits mit einer kleinen Fabrik, die jährlich 2500 PV-Module produziere, realisieren. Diese Erkenntnisse nahm Greenpeace in der Folge zum Anlass, um Kaufinteressenten für diese (Cyrus genannten) Anlagen zu suchen. Es wurden 4000 Kaufabsichtserklärungen gesammelt. Als Reaktion darauf schaltete Greenpeace Anzeigen in der FAZ und im Handelsblatt, um Unternehmer zu suchen. Fünf meldeten sich und zeigten Interesse entsprechende Anlagen aufzubauen. Die große Leistung von Greenpeace bestand v. a. in der Sensibilisierung der Nachfrager für PV. Aber nun war auch klar, dass PV-Anlagen doch kostengünstiger als gedacht zu produzieren seien und insbesondere mittelständische Unternehmen begannen sich für PV zu interessieren, wie etwa RAP Mikrosysteme GmbH aus Werni- 
gerode oder die Freiburger Solarfabrik (Ristau 1998: 57).Die neuen kleinen und mittelständischen PV-Firmen hatten sich von Beginn an auf netzgekoppelte Anlagen konzentriert. Sie produzierten Module, Aufständerungen für Dächer und Wechselrichter. Auf diese Weise hatten die von der Umweltbewegung angestoßenen Aktivitäten dazu geführt, den Pfad für die Innovationen in der Photovoltaik und die besondere Form ihrer Anwendung zu legen (vgl. Jacobsson/Lauber 2006: 266). Viele dieser kleinen Startups hatten ihren Ursprung in der PV-Forschung. Die enge Vernetzung von Wissenschaft, Umweltgruppen und kleinen, zu Beginn umwelt- und energiepolitisch hoch motivierten Firmengründern war im Fall der Photovoltaik in besonderem Maße gegeben.

Der zuvor bereits erwähnte institutionelle und infrastrukturelle Umbau auf dem Energiesektor hatte noch eine zweite Wirkung: Tradierte Entwicklungspfade wurden aufgebrochen und verändert, sodass eine generelle Diskussion über die Zukunft der deutschen und europäischen Energieversorgung angestoßen werden konnte. Die Umweltgruppen und ihre Aktivitäten nutzten die Situation, ebenso wie die Solarverbände, die sich Ende der 1990er Jahre gründeten und die junge PV-Industrie auf politischer Ebene vertraten. Als es dann im Jahre 1998 zur Gründung der Rot-Grünen Koalition kam, war das Window of Opportunity weit aufgestoßen: Die PV-Unterstützer-Koalition ergriff ihre Chance. Nun musste nicht mehr nur von außen Lobbyarbeit gemacht werden, sondern es ergab sich die Möglichkeit eines direkteren Einflusses auf die Politik und einer Institutionalisierung der Förderung Erneuerbarer Energien. Von der rot-grünen Koalition wurden zwei neue Politikinstrumente zur Förderung der Photovoltaik kreiert. Als Nachfolgeprogramm für das längst ausgelaufene 1000-Dächer-Programm wurde ein 100.000-Dächer-Programm verabschiedet. Das 1999 aufgelegte, über die KFW finanzierte Programm bot zinsvergünstigte Darlehen mit einer zehnjährigen Laufzeit an, von denen zwei Jahre tilgungsfrei waren. Lange hatte die PV-Industrie auf ein solches Signal gewartet. Im Jahre 2000 wurde dann schließlich ein neues Einspeisegesetz (Erneuerbare Energien-Gesetz, EEG) verabschiedet. Vor der Einführung des EEG sicherte sich die Bundesregierung eine breite Unterstützungs-Koalition, trotzdem versuchten einige der Energieversorgungsunternehmen, angeführt vom VDEW, nach wie vordas EEG zu verhindern. Als im Jahre 2003 das 100.000-Dächer-Programm auslief, wurde im Gegenzug mit einer ersten Novelle des EEG die Vergütung von Solarstrom aber noch erhöht, und auch als im Jahre 2005 die Rot-Grüne Koalition von einer Großen Koalition abgelöst wurde, wurde keine neue Richtung eingeschlagen. Mittlerweile sprach sich auch die CDU für Photovoltaik und die politische Unterstützung der weiteren Verbreitung von Photovoltaik aus. Insbesondere ostdeutsche Regionen profitierten durch die Ansiedlung der Photovoltaikindustrie, die ihren Ursprung in ersten Produktionsstätten im Jahre 1997 hat. Seitdem verlagerten eine Vielzahl der PV-Firmen ihre Produktionsstätten nach Ostdeutschland und auch ausländische Firmen siedelten sich dort an.

Mit dem Wechsel zu einer konservativ-liberalen Koalition änderten sich aber die Rahmenbedingungen für die deutsche Photovaltaikentwicklung. Das EEG wurde mehrfach verändert und verschlechterte signifikant die Rahmenbedingungen für die weitere Entwicklung der Photovoltaik. Energieversorger und andere interessierte organisierte Interessen forcierten eine Diskussion, die die finanzielle Förderung der PV als zu kostspielig bezeichnet. Im Gegenzug wurde die Laufzeit der Kernkraftwerke verlängert bis es nach dem Reaktorunfall in Fukushima wieder eine Kehrtwende gab und das Ende der Kernkraft proklamiert wurde, ohne dass sich deswegen die Rahmenbedingungen für die PV verbesserten. 


\section{Fazit: Governance von Innovationen im Energiesektor}

In unserem Beitrag haben wir die Entwicklung von zwei technischen Innovationen in drei Ländern betrachtet. Das Hauptaugenmerk lag dabei zum einen auf der Einbettung der technologischen Entwicklungen in die spezifischen nationalen Kontexte, die wir mit dem Begriff „Governance“ versuchten analytisch greifbar zu machen. Zum anderen lag der Schwerpunkt auf der Anwendung einer Prozessperspektive. Die Prozessperspektive war informiert durch die Theorie der strategischen Handlungsfelder (Neil Fligstein). Sie geht davon aus, dass ein Wandel der Governancestrukturen sich in der Veränderung der sie tragenden Akteurskonstellation niederschlagen muss. Die Veränderung von Akteurskonstellationenist ein im Prinzip konflikthafter Prozess.

Für den Fall Deutschland konnte gezeigt werden, dass die Entwicklung der Photovoltaik abhängig war von der Etablierung einer neuen Unterstützerkoalition, die gegen den Widerstand der etablierten Akteure und Interessen eine neue Form von Governance für die Förderung der Erneuerbaren Energien schuf. Die Unterstützerkoalition erweiterte sich sukzessive, besteht aber nach wie vor aus einer breiten, interessenspolitisch diffusen Gruppe von Akteuren, der es nur punktuell gelingt, gemeinsame Interessen zu formulieren. Wir beobachten hier die Entwicklung einer Governancestruktur quasi „von unten“ deren dauerhafte Existenz aber von Entscheidungen „,von oben“ abhängig ist. Zumindest zum jetzigen Zeitpunkt hat sich eine Governancestruktur ausdifferenziert und die Frage einer grundlegenderen Veränderung bzw. Transformation des Energiesystems ist offen (Ausdifferenzierung bis Transformation).

Die CCS-Technologie hingegen sollte von den etablierten Netzwerken aus Energieversorgern, Wissenschaft, Kraftwerkskomponentenherstellern und Politik quasi von oben exekutiert werden und versuchte eine neue technische Option auch gegen Widerstand von gesellschaftlichen Gruppen durchzusetzen. Das Scheitern der CCS Entwicklung wird deutlich in den Organisationserfolgen der Gegner der CCS-Entwicklung und der mangelnden Fähigkeit der etablierten Akteure diese Gruppen (wie z. B. in Norwegen) zu kooptieren (unorganisierter sozialer Raum).

In Norwegen wurde die CCS Entwicklung von einer breiten Gruppe von Akteuren vorangetrieben, die zunächst nicht zum etablierten Stromerzeugungssystem gehörten. Über geschickte Kooptationsstrategien hat sich aber eine breite Koalition von Akteuren aus dem ursprünglichen und benachbarten Feldern zusammengetan, um CCS voranzutreiben (Transformation).

Die Photovoltaikentwicklung ist auf der einen Seite erfolgreich gewesen, weil die Zielsetzungen der etablierten Hauptakteure sich im Wesentlichen realisieren ließen. Ziel war es zum einen die Photovoltaikentwicklung voranzutreiben, ohne die Position der etablierten Akteure zu gefährden. Hierzu stand im Vordergrund, ein exportfähige Technologie zu entwickeln, die referenzmäßig auch in Japan angewandt wurde. Zum anderen sollten aber die vorhandenen Strukturen in Japan und die Interessen der dominanten Akteure des Energiesystems nicht in Frage gestellt werden. Dies hat zu einer vergleichbaren konstanten, aber in der Summe eher unbedeutenden Entwicklung der PV in Japan geführt (Konstanz der Governancestruktur). 


\section{Literatur}

Alphen, van, Claas/Ruijven, van, Jochem/Kasa, Sjur/Hekkert, Marko/Turkenburg, Wim (2009):

The performance of the Norwegian carbon dioxide, capture and storage innovation system, Energy Policy 37(1): 43-55.

Anderson, Kent/Nottage, Luke R./Wolff, Leon T./Yamanaka, William (2008): Japan's Gradual Transformation in Corporate Governance. Research Paper No. 08/29. Sydney: Sydney Law School.

Benz, Arthur/Lütz, Susanne/Schimank, Uwe/Simonis, Georg (Hrsg.) (2007): Handbuch Governance. Theoretische Grundlagen und empirische Anwendungsfelder. Wiesbaden: VS Verlag.

Benz, Arthur/Dose, Nicolai (2010): Governance - Regieren in komplexen Regelsystemen: Eine Einführung. 2. veränderte und aktualisierte A. Wiesbaden: VS Verlag.

Boyer, Robert (2003): The Embedded Innovation Systems of Germany and Japan: Distinctive Features and Futures, in: Yamamura, Kozo/Streeck, Wolfgang (Hrsg.): The End of Diversity? Prospects for German and Japanese Capitalism. Cornell: Cornell University Press: 147-183.

Fligstein, Neil/McAdam, Doug 2011: Toward a General Theory of Strategic Action Fields. Sociological Theory 29(1), 1-26.

Jacobsson, Staffan/Lauber, Volkmar (2006): The politics and policy of energy system transformation - explaining the German diffusion of renewable energy technology, Energy Policy 343: 256-276.

Mayntz, Renate (2004): GovernanceTheory als fortentwickelte Steuerungstheorie? MPIfG Working Paper 04/1. Max Planck Institut für Gesellschaftsforschung, Köln.

Meyer, John W./Rowan, Brian (1977): Institutionalized Organizations: Formal Structure as Myth and Ceremony. American Journal of Sociology 88, 340-63.

Pahle, Michael (2010): Germany's dash for coal: Exploring drivers and factors, Energy Policy 38(7): 3431-3442.

Ristau, Oliver (1998): Die solare Standortfrage. Der technologische Wettstreit zwischen den USA, Japan und Deutschland, Bad Oeynhausen: Bröer-und-Witt-Solarthemen.

Rogge, Karoline/Hoffmann, Volker (2009): The impact of the EU ETS on the sectoral innovation system for power generation technologies - Findings for Germany, Paper submitted to DIME Workshop on „Environmental Innovation, Industrial Dynamics and Entrepreneurship, May 10-12, 2009, Utrecht, Netherlands.

Schulz, Marlen/Scheer, Dirk/Wassermann, Sandra (2010): Neue Technik, alte Pfade? Zur Akzeptanz der $\mathrm{CO}_{2}$-Speicherung in Deutschland. In: GAIA 19(4): 287-296.

Shum, Kwok L./Watanabe, Shiro (2009): An innovation management approach for renewable energy development - the case of solar photovoltaic (PV) development. EnergyPolicy37(9): 3535-3544.

UBA - Umweltbundesamt (2011): Strommix in Deutschland, in: http://www.umweltbundesamt.de/ energie/archiv/strommix-karte.pdf, zugegriffen am 10. September 2011.

Victor, David G. (2002): Electric Power, in: Steil, Benn et al (Hrsg.) Technological Innovation and Economic Performance. Princeton: Princeton University Press, 385-415. 
Vogel, Steven K. (2006): Japan Remodeled. How government and industry are reforming Japanese capitalism, Ithaca: Cornell University Press Weimer-Jehle,Wolfgang/Wassermann, Sandra/ Fuchs, Gerhard (2010): Erstellung von Energie- und Innovationsszenarien mit der CrossImpact-Bilanzanalyse: Internationalisierung von Innovationstrategien im Bereich der Kohlekraftwerkstechnologie. In: Institut für Elektrizitätswirtschaft und Energieinnovation (Hrsg.), 11. Symposium Energieinnovation. Alte Ziele - Neue Wege. Wien.

Welt Online (1995): Eine Lobby für die Sonnenergie, http:/www.welt.de/print-welt/article663558/ Eine_Lobby_fuer_die_Sonnenergie.html, zugegriffen am 11. September, 2007. 


\section{Bisher erschienene manu:scripte}

ITA-0I-0I Gunther Tichy, Walter Peissl (12/2001): Beeinträchtigung der Privatsphäre in der Informationsgesellschaft. <www.oeaw.ac.at/ita/pdf/ita_0l_0l.pdf>

ITA-0I-02 Georg Aichholzer (12/2001): Delphi Austria: An Example of Tailoring Foresight to the Needs of a Small Country. <www.oeaw.ac.at/ita/pdf/ita_0I_02.pdf>

ITA-0I-03 Helge Torgersen, Jürgen Hampel (12/2001): The Gate-Resonance Model: The Interface of Policy, Media and the Public in Technology Conflicts. <www.oeaw.ac.at/ita/pdf/ita_0I_03.pdf>

ITA-02-0I Georg Aichholzer (01/2002): Das ExpertInnen-Delphi: Methodische Grundlagen und Anwendungsfeld „Technology Foresight“. <www.oeaw.ac.at/ita/pdf/ita_02_0l.pdf>

ITA-02-02 Walter Peissl (01/2002): Surveillance and Security - A Dodgy Relationship. $<w w w$. oeaw.ac.at/ita/pdf/ita_02_02.pdf>

ITA-02-03 Gunther Tichy (02/2002): Informationsgesellschaft und flexiblere Arbeitsmärkte. $<w w w . o e a w . a c . a t / i t a / p d f / i t a \_02 \_03 . p d f>$

ITA-02-04 Andreas Diekmann (06/2002): Diagnose von Fehlerquellen und methodische Qualität in der sozialwissenschaftlichen Forschung. <www.oeaw.ac.at/ita/pdf/ita_02_04.pdf>

ITA-02-05 Gunther Tichy (10/2002): Over-optimism Among Experts in Assessment and Foresight.

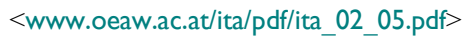

ITA-02-06 Hilmar Westholm (12/2002): Mit eDemocracy zu deliberativer Politik? Zur Praxis und Anschlussfähigkeit eines neuen Mediums. <www.oeaw.ac.at/ita/pdf/ita_02_06.pdf>

ITA-03-0I Jörg Flecker und Sabine Kirschenhofer (01/2003): IT verleiht Flügel? Aktuelle Tendenzen der räumlichen Verlagerung von Arbeit. <www.oeaw.ac.at/ita/pdf/ita_03_0l.pdf>

ITA-03-02 Gunther Tichy (11/2003): Die Risikogesellschaft - Ein vernachlässigtes Konzept in der europäischen Stagnationsdiskussion. < www.oeaw.ac.at/ita/pdf/ita_03_02.pdf>

ITA-03-03 Michael Nentwich (11/2003): Neue Kommunikationstechnologien und Wissenschaft - Veränderungspotentiale und Handlungsoptionen auf dem Weg zur Cyber-Wissenschaft. <www.oeaw.ac.at/ita/pdf/ita_03_03.pdf>

ITA-04-0I Gerd Schienstock (1/2004): Finnland auf dem Weg zur Wissensökonomie - Von Pfadabhängigkeit zu Pfadentwicklung. <www.oeaw.ac.at/ita/pdf/ita_04_0I.pdf>

ITA-04-02 Gunther Tichy (6/2004): Technikfolgen-Abschätzung: Entscheidungshilfe in einer komplexen Welt. $<$ www.oeaw.ac.at/ita/pdf/ita_04_02.pdf>

ITA-04-03 Johannes M. Bauer (11/2004): Governing the Networks of the Information Society - Prospects and limits of policy in a complex technical system. <www.oeaw.ac.at/ita/pdf/ita_04_03.pdf>

ITA-04-04 Ronald Leenes (12/2004): Local e-Government in the Netherlands: From Ambitious Policy Goals to Harsh Reality. <www.oeaw.ac.at/ita/pdf/ita_04_04.pdf>

ITA-05-0I Andreas Krisch (01/2005): Die Veröffentlichung des Privaten - Mit intelligenten Etiketten vom grundsätzlichen Schutz der Privatsphäre zum Selbstschutz-Prinzip. <www.oeaw.ac.at/ita/pdf/ita_05_0I.pdf >

ITA-05-02 Petra Grabner (12/2005): Ein Subsidiaritätstest - Die Errichtung gentechnikfreier Regionen in Österreich zwischen Anspruch und Wirklichkeit. <www.oeaw.ac.at/ita/pdf/ita_05_02.pdf >

ITA-05-03 Eva Buchinger (12/2005): Innovationspolitik aus systemtheoretischer Sicht - Ein zyklisches Modell der politischen Steuerung technologischer Innovation. <www.oeaw.ac.at/ita/pdf/ita_05_03.pdf $>$

ITA-06-0I Michael Latzer (06/2006): Medien- und Telekommunikationspolitik: Unordnung durch Konvergenz Ordnung durch Mediamatikpolitik. <epub.oeaw.ac.at/ita/ita-manuscript/ita_06_0I.pdf>

ITA-06-02 Natascha Just, Michael Latzer, Florian Saurwein (09/2006): Communications Governance: Entscheidungshilfe für die Wahl des Regulierungsarrangements am Beispiel Spam. <epub.oeaw.ac.at/ita/ita-manuscript/ita_06_02.pdf>

ITA-06-03 Veronika Gaube, Helmut Haberl (10/2006): Sozial-ökologische Konzepte, Modelle und Indikatoren nachhaltiger Entwicklung: Trends im Ressourcenverbrauch in Österreich. <epub.oeaw.ac.at/ita/ita-manuscript/ita_06_03.pdf>

ITA-06-04 Maximilian Fochler, Annina Müller (11/2006): Vom Defizit zum Dialog? Zum Verhältnis von Wissenschaft und Öffentlichkeit in der europäischen und österreichischen Forschungspolitik. <epub.oeaw.ac.at/ita/ita-manuscript/ita_06_04.pdf>

ITA-06-05 Holger Floeting (11/2006): Sicherheitstechnologien und neue urbane Sicherheitsregimes. <epub.oeaw.ac.at/ita/ita-manuscript/ita_06_05.pdf>

ITA-06-06 Armin Spök (12/2006): From Farming to „Pharming” - Risks and Policy Challenges of Third Generation GM Crops. <epub.oeaw.ac.at/ita/ita-manuscript/ita_06_06.pdf>

ITA-07-0I Volker Stelzer, Christine Rösch, Konrad Raab (3/2007): Ein integratives Konzept zur Messung von Nachhaltigkeit - das Beispiel Energiegewinnung aus Grünland. $<$ epub.oeaw.ac.at/ita/ita-manuscript/ita_07_01.pdf> 
ITA-07-02 Elisabeth Katzlinger (3/2007): Big Brother beim Lernen: Privatsphäre und Datenschutz in Lernplattformen. $<$ epub.oeaw.ac.at/ita/ita-manuscript/ita_07_02.pdf>

ITA-07-03 Astrid Engel, Martina Erlemann (4/2007): Kartierte Risikokonflikte als Instrument reflexiver Wissenspolitik. <epub.oeaw.ac.at/ita/ita-manuscript/ita_07_03.pdf>

ITA-07-04 Peter Parycek (5/2007): Gläserne Bürger - transparenter Staat? Risiken und Reformpotenziale des öffentlichen Sektors in der Wissensgesellschaft. <epub.oeaw.ac.at/ita/ita-manuscript/ita_07_04.pdf>

ITA-07-05 Helge Torgersen (7/2007): Sicherheitsansprüche an neue Technologien - das Beispiel Nanotechnologie. <epub.oeaw.ac.at/ita/ita-manuscript/ita_07_05.pdf>

ITA-07-06 Karen Kastenhofer (9/2007): Zwischen „schwacher“ und „starker“ Interdisziplinarität. Die Notwendigkeit der Balance epistemischer Kulturen in der Sicherheitsforschung zu neuen Technologien.

<epub.oeaw.ac.at/ita/ita-manuscript/ita_07_06.pdf>

ITA-07-07 Ralf Lindner, Michael Friedewald (9/2007): Gesellschaftliche Herausforderungen durch ,intelligente Umgebungen. Dunkle Szenarien als TA-Werkzeug. <epub.oeaw.ac.at/ita/ita-manuscript/ita_07_07.pdf>

ITA-07-08 Alfons Bora (11/2007): Die disziplinären Grundlagen der Wissenschaft. <epub.oeaw.ac.at/ita/ita-manuscript/ita_07_08.pdf>

ITA-08-0I Alexander Degelsegger (5/2008): „Frames“ in sozialwissenschaftlichen Theorieansätzen. Ein Vergleich aus der Perspektive der Technikforschung. <epub.oeaw.ac.at/ita/ita-manuscript/ita_08_0I.pdf>

ITA-08-02 Jens Hoff (11/2008): Can The Internet Swing The Vote? Results from a study of the 2007 Danish parliamentary election. <epub.oeaw.ac.at/ita/ita-manuscript/ita_08_02.pdf>

ITA-09-0I Georg Aichholzer, Doris Allhutter (2/2009): e-Participation in Austria: Trends and Public Policies. <epub.oeaw.ac.at/ita/ita-manuscript/ita_09_0I.pdf>

ITA-09-02 Michael Nentwich (11/2009): Cyberscience 2.0 oder 1.2? Das Web 2.0 und die Wissenschaft. <epub.oeaw.ac.at/ita/ita-manuscript/ita_09_02.pdf>

ITA-09-03 Hilmar Westholm (12/2009): Wandel der Formen politischer Partizipation und der Beitrag des Internet. Schlussfolgerungen aus Bevölkerungsbefragungen in Deutschland. <epub.oeaw.ac.at/ita/ita-manuscript/ita_09_03.pdf>

ITA-10-0I Iris Eisenberger (12/2010): Kleine Teile, große Wirkung? Nanotechnologieregulierung in der Europäischen Union. <epub.oeaw.ac.at/ita/ita-manuscript/ita_I0_0I.pdf>

ITA-10-02 Alexander Degelsegger and Helge Torgersen (12/2010): Instructions for being unhappy with PTA. The impact on PTA of Austrian technology policy experts' conceptualisation of the public. <epub.oeaw.ac.at/ita/ita-manuscript/ita_10_02.pdf>

ITA-10-03 Ernest Braun (12/2010): The Changing Role of Technology in Society. <epub.oeaw.ac.at/ita/ita-manuscript/ita_10_03.pdf>

ITA-I0-04 Fritz Betz (12/2010): E-Partizipation und die Grenzen der Diskursethik. <epub.oeaw.ac.at/ita/ita-manuscript/ita_10 04.pdf>

ITA-I I-0I Peter Parycek, Judith Schoßböck (1/2011): Transparency for Common Good. Offener Zugang zu Information im Kontext gesellschaftlicher und strategischer Spannungsfelder. <epub.oeaw.ac.at/ita/ita-manuscript/ita_II_0I.pdf>

ITA-II-02 Georg Aichholzer und Doris Allhutter (6/2011): Online forms of political participation and their impact on democracy. <epub.oeaw.ac.at/ita/ita-manuscript/ita_II_02.pdf>

ITA-I I-03 Mahshid Sotoudeh, Walter Peissl, Niklas Gudowsky, Anders Jacobi (12/2011): Long-term planning for sustainable development. CIVISTI method for futures studies with strong participative elements. <epub.oeaw.ac.at/ita/ita-manuscript/ita_II 03.pdf>

ITA-I2-0I Xiao Ming (1/2012): e-Participation in Government Decision-Making in China. Reflections on the Experience of Guangdong Province. <epub.oeaw.ac.at/ita/ita-manuscript/ita_12_01.pdf>

ITA-12-02 Stephan Bröchler, Georg Aichholzer, Petra Schaper-Rinkel (Hrsg.) (7/2012): Theorie und Praxis von Technology Governance. <epub.oeaw.ac.at/ita/ita-manuscript/ita_12_02_Sondernummer.pdf> 